\title{
Teoria não comutativa de integração e dinâmica hiperbólica
}





\section{Teoria não comutativa de integração e dinâmica hiperbólica ${ }^{1}$}

\section{Gabriel Elias Mantovani}

Orientador: Prof. Dr. Ali Tahzibi

Dissertação apresentada ao Instituto de Ciências Matemáticas e de Computação - ICMC-USP, como parte dos requisitos para obtenção do título de Mestre em Ciências

- Matemática. VERSÃO REVISADA

\section{USP - São Carlos}

Agosto/2013

\footnotetext{
${ }^{1}$ Este trabalho teve suporte financeiro da FAPESP
} 
Ficha catalográfica elaborada pela Biblioteca Prof. Achille Bassi e Seção Técnica de Informática, ICMC/USP, com os dados fornecidos pelo(a) autor(a)

M293t $\begin{aligned} & \text { Mantovani, Gabriel Elias } \\ & \text { Teoria não comutativa de integração e dinâmica } \\ & \text { hiperbólica / Gabriel Elias Mantovani; orientador } \\ & \text { Ali Tahzibi. -- São Carlos, 2013. } \\ & 59 \text { p. } \\ & \text { Dissertação (Mestrado - Programa de Pós-Graduação } \\ & \text { em Matemática) -- Instituto de Ciências Matemáticas } \\ & \text { e de Computação, Universidade de São Paulo, 2013. } \\ & \text { 1. desintegração. 2. dinâmica hiperbólica. 3. } \\ & \text { medida SRB. 4. grupoide. 5. teoria não comutativa } \\ & \text { de integração. I. Tahzibi, Ali, orient. II. Título. }\end{aligned}$


Aos meus pais. 
"No, no! The adventures first, explanations take such a dreadful time."

Lewis Carroll, Alice in Wonderland 


\section{Agradecimentos}

Ao meu orientador Ali Tahzibi, pelo apoio, atenção, pelos momentos de discussão frutífera e pelo enriquecimento da minha experiência matemática.

Aos meus pais, Elenice e Luiz Eduardo, e a minha irmã Sarah, por terem sempre me apoiado nas minhas decisões, e por terem contribuído de forma fundamental na minha formação pessoal.

Aos colegas de mestrado Jorge Parejas e Laís Oliveira pelo suporte fundamental durante o primeiro semestre do mestrado.

À FAPESP pelo suporte financeiro. 


\section{Resumo}

\section{MANTOVANI, G . E. Teoria não comutativa de integração e dinâmica hiperbólica.}

2013. 71 f. Dissertação (Mestrado) - Instituto de Ciências Matemáticas e de Computação, Universidade de São Paulo, São Carlos, 2013.

Uma das caracterizações de medidas SRB é a de que a sua desintegração em relação as partições mensuráveis subordinadas as variedades instáveis são absolutamente continuas com respeito a medida Lebesgue nestas mesmas variedades. Este trabalho segue os passos de Segert [16] ao analisar a aplicabilidade da teoria de integração não comutativa de Alain Connes ao estudo de medidas SRB de sistemas dinâmicos hiperbólicos do tipo $(M, f)$ com $M$ uma variedade compacta e $f$ um difeomorfismo $C^{2}$. Nesta dissertação é realizada a demonstração do teorema da desintegração de Rokhlin utilizando o conceito de esperança condicional. É fornecida uma introdução a teoria de integração não comutativa de Alain Connes. E apresenta-se uma injeção entre medidas SRB de sistemas dinâmicos hiperbólicos e as medidas transversas definidas sobre este sistema.

Palavras-chave: desintegração, grupoides, teoria não comutativa de integração, dinâmica hiperbólica, medidas SRB. 


\section{Abstract}

\section{MANTOVANI, G. E. Non commutative integration theory and hiperbolic dynamic.}

2013. 71 f. Dissertação (Mestrado) - Instituto de Ciências Matemáticas e de Computação, Universidade de São Paulo, São Carlos, 2013.

A characterization of SRB measures is that its disintegration in relation to measurable partitions subordinate to unstable manifolds is absolutely continous with respect to the Lesbesgue measures in the same manifolds. This work follows the footsteps of Segert [16] in the study of the applicability of the non commutative integration theory of Alain Connes to the analysis of SRB measures for hyperbolic dynamical systems of the type $(M, f)$ with $M$ a compact manifold and $f$ a $C^{2}$ diffeomorphism. In this work the proof of Rokhlin's disintegration theorem is presented using the concept of conditional expectation. An introduction to the theory of non commutative integration of Alain Connes is provided. It's shown the existence of a injection between SRB measures in hyperbolic dynamical systems and transverse measures defined on this system.

Keywords: disintegration, non commutative integration theory, hyperbolic dynamic, SRB measures. 


\section{Sumário}

Lista de Abreviaturas e Símbolos $\quad$ ix

1 Introdução $\quad 1$

2 Medida, Desintegração e Dinâmica $\quad 3$

2.1 Espaços de Medida . . . . . . . . . . . . . . . . . . . . . . . . 3

2.2 Partições . . . . . . . . . . . . . . . . . . . . . . 6

2.3 O Teorema da Desintegração de Rokhlin . . . . . . . . . . . . . . . . . . 10

2.4 Sistemas Dinâmicos Uniformemente Hiperbólicos ． . . . . . . . . . . . 16

2.5 Sistemas Dinâmicos Não Uniformemente Hiperbólicos . . . . . . . . . . . . . 19

3 Introdução a Teoria Não Comutativa de Integração 21

3.1 Grupoides . . . . . . . . . . . . . . . . . . . . . . . 21

3.2 Grupoides de Medida . . . . . . . . . . . . . . . . . . . . . . . 24

3.3 Kernel . . . . . . . . . . . . . . . . . . . . . . 25

3.4 Funções Transversas . . . . . . . . . . . . . . . . . . . . . . . 31

3.5 Medidas Transversas . . . . . . . . . . . . . . . . . . . . . . 34

3.6 Exemplos . . . . . . . . . . . . . . . . . . . . . 39

4 Teoria de Integração não Comutativa e Sistemas Dinâmicos 45

4.1 Funções Transversas de Riemann . . . . . . . . . . . . . . . . . . . . . . . 46

4.2 Homomorfismo Jacobiano . . . . . . . . . . . . . . . . . . . 50

4.3 Medidas Transversas e Medidas SRB . . . . . . . . . . . . . . 53

4.4 Comentários Finais . . . . . . . . . . . . . . . . . . . . . 55

$\begin{array}{ll}\text { Referências Bibliográficas } & 57\end{array}$

$\begin{array}{ll}\text { Índice Remissivo } & 59\end{array}$ 


\section{Lista de Abreviaturas e Símbolos}

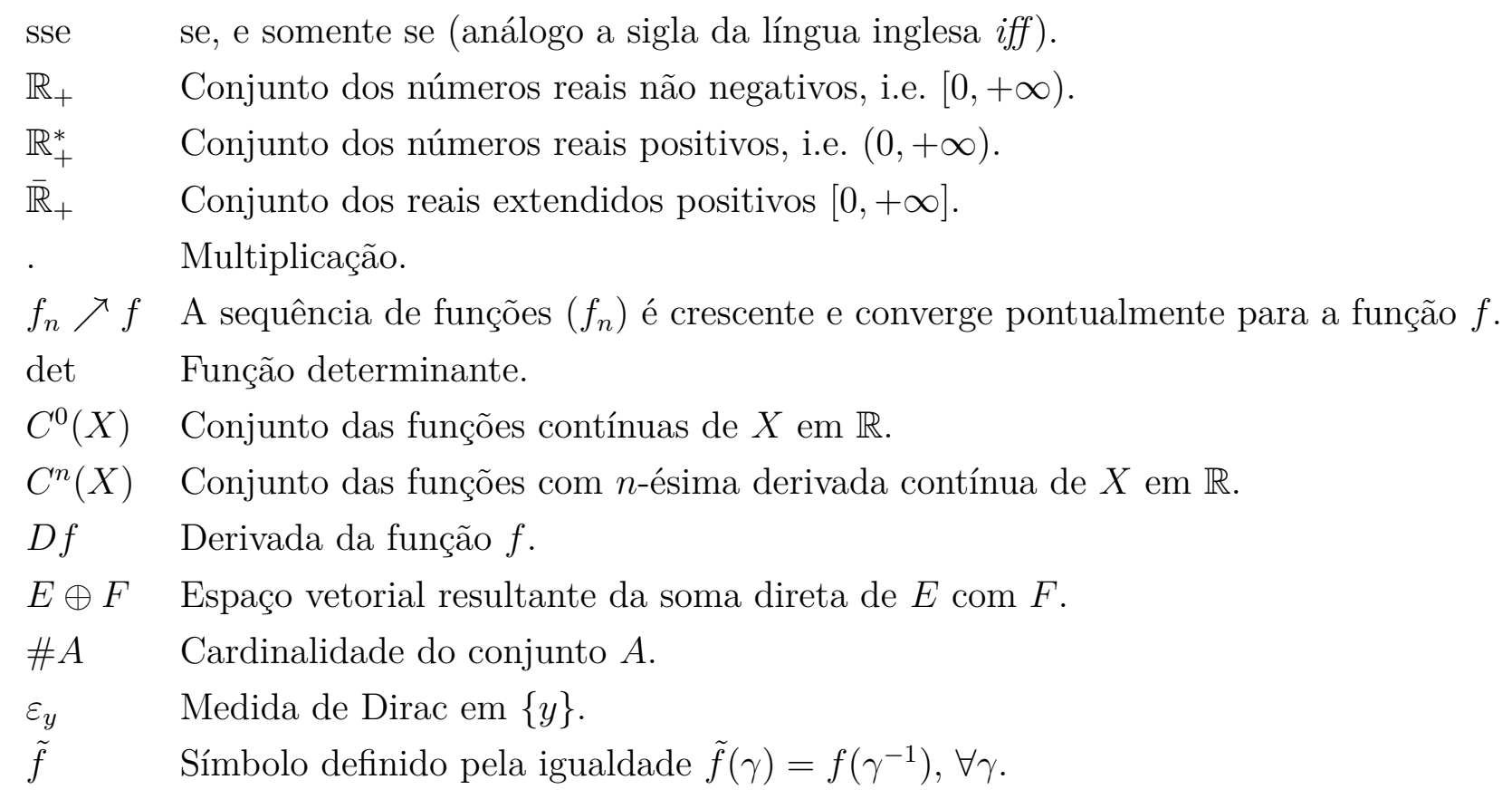

\section{Capítulo 2.}

$\begin{array}{ll}\sigma(W) & \sigma \text {-álgebra gerada pelo conjunto } W, \text { p. } 3 . \\ \chi_{A} & \text { Função característica do conjunto } A, \text { p. } 5 . \\ \mathbb{X} \otimes \mathbb{Y} & \text { Produto das } \sigma \text {-álgebras } \mathbb{X} \text { e } \mathbb{Y}, \text { p. } 4 . \\ A=B \bmod 0 & \text { Existe conjunto de medida total } F \text { tal que } A \cap F=B \cap F, 5 . \\ \text { TCM } & \text { Teorema da Convergência Monótona, p.5. } \\ \text { TCD } & \text { Teorema da convergência Dominada, p.6. } \\ \mu \ll \nu & \text { A medida } \mu \text { é absolutamente contínua com respeito a medida } \nu, \text { p. } 6 . \\ \mathcal{P} \preceq \mathcal{Q} & \text { A partição } \mathcal{Q} \text { é mais refinada que a partição } \mathcal{P}, \text { p. } 7 . \\ \mathcal{P} \vee \mathcal{Q} & \text { Partição menos refinada que refina } \mathcal{P} \text { e } \mathcal{Q}, \text { p. } 7 . \\ W^{u}(x) & \text { Conjunto globalmente instável, p. } 20 . \\ W^{s}(x) & \text { Conjunto globalmente estável, p. } 20 .\end{array}$




\section{Capítulo 3.}

$G^{(0)} \quad$ Conjunto dos objetos do grupoide $G$, p. 21.

$G^{(2)} \quad$ Conjunto dos elementos multiplicáveis do grupoide $G$, p.21.

$\mathbb{1}_{x} \quad$ Homomorfismo identidade de $x$ em $x$, p. 21.

$r, s$, inv Funções alvo, fonte e inversão em grupoides, p. 22.

$G_{A} \quad$ Subgrupoide de $G$ obtido pela restrição de $G^{(0)}$ a $A$, p. 22.

$\mathcal{F}^{+}(G) \quad$ Espaço das funções mensuráveis de $G \rightarrow[0, \infty)$, p. 25.

$\overline{\mathcal{F}}^{+}(G) \quad$ Espaço das funções mensuráveis de $G \rightarrow[0, \infty]$, p. 25.

$\mathcal{C}^{+} \quad$ Espaço dos kernels próprios de um dado grupoide, p. 27.

$R_{\nu}, L_{\nu} \quad$ p. 27 .

$\nu * f \quad$ Convolução de kernel por função, p.27.

$f * g \quad$ Convolução de $f$ por $g$ segundo o kernel $\nu$, p. 27.

$\nu * \mu \quad$ Convolução de kernel por kernel, p.28.

$S_{\nu} \quad$ Suporte do kernel $\nu$, p. 30.

$\mathcal{E}^{+} \quad$ Espaço das funções transversas próprias de um dado grupoide, p. 33.

$\delta \quad$ Função modular, p. 34 .

$\Delta \quad$ Medida transversa, p. 34.

$\Delta_{\nu} \quad$ Medida induzida pela função transversa $\nu$, p. 35.

$\otimes \quad$ Produto tensorial, p. 40.

\section{Capítulo 4.}

$G_{u} \quad$ Grupoide instável, p. 45.

$\delta_{u} \quad$ Homomorfismo Jacobiano, p. 51. 


\section{Capítulo 1}

\section{Introdução}

No estudo de um sistema dinâmico certas probabilidades invariantes são especialmente úteis, entre estas se destacam as medidas físicas ou SRB, denominadas desta forma em homenagem aos pesquisadores pioneiros em seu estudo, Sinai, Ruelle e Bowen. Esta dissertação se baseia na seguinte forma de se caracterizar a medida SRB de um sistema dinâmico: uma medida invariante em um sistema dinâmico é uma medida SRB se quase todos elementos de sua desintegração em relação a uma partição subordinada as folheações instáveis são absolutamente contínuas com respeito a medida de Lebesgue nesta folhação instável.

A desintegração de uma probabilidade em relação a uma partição é uma família de probabilidades indexada pelos elementos da partição, de tal sorte que cada probabilidade tem seu suporte no respectivo elemento da partição; e é possível reobter a probabilidade original a partir de um processo de integração desta família. Para um exemplo considere a partição por linhas horizontais do quadrado $[0,1] \times[0,1]$, para cada átomo da partição (i.e. cada linha horizontal) associa-se a probabilidade boreliana usual unidimencional. Temse portanto que o suporte de cada probabilidade está em sua respectiva partição; e pelo teorema de Fubini pode-se reobter a probabilidade boreliana do quadrado atrávez de uma integral.

Uma partição é dita mensurável se pode ser obtida por um processo de limite decrescente, i.e., que os átomos da partição podem ser escritos como uma interseção decrescente enumerável de conjuntos mensuráveis. O teorema da desintegração de Rohklin garante que um espaço métrico compacto dotado de uma medida de probabilidade boreliana sempre possui uma desintegração em relação a uma partição mensurável.

Esta dissertação segue os passos de Segert [16] que buscou estudar as medidas condicionais (desintegração) de um sistema dinâmico sob a luz da teoria não comutativa de integração de Alain Connes. Segert encontrou uma injeção entre as medidas transversas definidas em um grupoide associado as variedades instáveis de um sistema dinâmico e as medidas SRB deste sistema.

Este trabalho foi escrito com o objetivo de fazer uma análise sobre mensurabilidade de partições, desintegração de medidas no contexto de sistemas hiperbólicos, teoria não commutativa de integração de A. Connes, e explorar possíveis conexões entre estes temas. A referência principal para este estudo é a tese de doutorado de Jan Segert [16]. É digno de 
nota que apesar desta dissertação ter como foco sistemas dinâmicos, este trabalho é mais fortemente relacionado a Teoria da Medida do que a sistemas dinâmicos em si. A seguir é realizada uma descrição capítulo a capítulo.

Na primeira seção do capítulo 2 é apresentada uma breve revisão dos conceitos fundamentais de teoria da medida que serão utilizados ao longo da dissertação. Na segunda seção é apresentado o conceito de partições de forma rigorosa, e as partições mensuráveis são definidas. A terceira seção traz a prova do teorema da desintegração de Rokhlin. E finalmente nas duas últimas seções é realizada uma breve revisão dos conceitos de dinâmica utilizados na dissertação, tais conceitos são apresentados utilizando as noções de medidas condicionais e partições subordinadas. Este capítulo tem como principais referências [14] e [6].

No capítulo 3 é fornecida uma introdução a Teoria de Integração Não Comutativa de Alain Connes. Ao inicio do capítulo se define o conceito de grupoide e grupoide mensurável. Nas três seções seguintes são apresentados os conceitos, relativamente abstratos, de kernel, função transversa e medida transversa. Com o objetivo de facilitar o entendimento de tais conceitos o capítulo termina com uma seção composta exclusivamente de exemplos. Este capítulo tem como principais referências [7] e [3].

O último capítulo traz uma uma série de resultados de [16] que apresentam uma unificação dos conceitos trabalhados, e abrem caminho para uma possível linha de estudo sobre medidas SRB. 


\section{Capítulo 2}

\section{Medida, Desintegração e Dinâmica}

O objetivo principal deste capítulo é compreender a seguinte caracterização de medidas SRB: para um sistema hiperbólico as medidas SRB são aquelas cuja desintegração em relação as partições mensuráveis subordinadas as variedades instáveis deste sistema são absolutamente continuas com respeito a medida Lebesgue nestas mesmas variedades. Com este objetivo em mente na primeira seção é apresentada uma breve revisão dos conceitos fundamentais de teoria da medida que serão utilizados ao longo da dissertação. Na segunda seção é apresentado o conceito de partição de um espaço mensurável de forma rigorosa, e o conceito de partição mensurável é definido. A terceira seção traz a prova do teorema da desintegração de Rokhlin. E finalmente nas últimas duas seções é realizada uma breve revisão dos conceitos de dinâmica utilizados na dissertação. Este capítulo tem como principais referências [14] e [6].

\subsection{Espaços de Medida}

Seja $X$ um conjunto, uma $\sigma$-álgebra $\mathbb{X}$ de $X$ é um subconjunto do conjunto das partes de $X, 2^{X}$, que é fechado por união enumerável, por complementariedade e possui $X$ como um elemento. Assim $\mathbb{X} \subset 2^{X}$ é $\sigma$-álgebra sobre $X$ se satisfaz :

1. $X \in \mathbb{X}$

2. Se $A \in \mathbb{X}$ então $X \backslash A \in \mathbb{X}$;

3. Se $\left(A_{n}\right)_{n \in \mathbb{N}}$ é sequência de elementos de $\mathbb{X}$ então $\bigcup_{n \in \mathbb{N}} A_{n} \in \mathbb{X}$.

Neste caso denomina-se a dupla ordenada $(X, \mathbb{X})$ de espaço mensurável, e todo conjunto $A \in \mathbb{X}$ se diz mensurável .

Dado conjunto $W \subset 2^{X}$ define-se a $\sigma$-álgebra gerada por $W, \sigma(W)$ como sendo a menor $\sigma$-álgebra que contém $W$, i.e., se $\mathbb{X}$ é $\sigma$-álgebra em $X$ tal que $W \subset \mathbb{X}$ então $\sigma(W) \subset \mathbb{X}$ 1. A existência de $\sigma(W)$ é garantida pelo fato de que $2^{X}$ é uma $\sigma$-álgebra e de que interseções

${ }^{1} \mathrm{~A} \sigma$-álgebra foi definida de forma não construtiva. É um fato importante mas comumente negligênciado que $\sigma(W)$ pode ser obtida de forma construtiva [4]. 
arbitrárias de $\sigma$-álgebras são $\sigma$-álgebras. Assim pode-se escrever

$$
\sigma(W)=\bigcap_{W \subset \mathbb{A}, \mathbb{A} \text { é } \sigma \text {-álgebra }} \mathbb{A} .
$$

Uma $\sigma$-álgebra $\mathbb{X}$ é dita enumeravelmente gerada caso exista um conjunto $W$ enumerável tal que $\mathbb{X}=\sigma(W)$.

Sejam $(X, \mathbb{X}),(Y, \mathbb{Y})$ espaços mensuráveis, uma função $f:(X, \mathbb{X}) \rightarrow(Y, \mathbb{Y})$ é dita função mensurável se $A \in \mathbb{Y} \Rightarrow f^{-1}(A) \in \mathbb{X}$, i.e., a imagem inversa de cada conjunto mensurável de $\mathbb{Y}$ é um conjunto mensurável de $\mathbb{X}$. Caso $\mathbb{Y}=\sigma(B)$ para algum $B \subset 2^{Y}$, $f$ é mensurável sse $f^{-1}(A) \in \mathbb{X}, \forall A \in B$.

Seja $Y$ um conjunto e $g:(X, \mathbb{X}) \rightarrow Y$ uma função, denomina-se a $\sigma$-álgebra induzida em $Y$ pela função $g$ o conjunto $\mathbb{Y}=\left\{E \subset Y \mid g^{-1}(E) \in \mathbb{X}\right\}$. É de fácil verificação que $\mathbb{Y}$ é $\sigma$-álgebra e que é a menor $\sigma$-álgebra que torna $g:(X, \mathbb{X}) \rightarrow(Y, \mathbb{Y})$ uma função mensurável . Uma bijeção $h:(X, \mathbb{X}) \rightarrow(Y, \mathbb{Y})$ é chamada de isomorfismo mensurável se tanto $h$ como $h^{-1}$ são funções mensuráveis.

Seja $(X, \tau)$ um espaço topológico ([13], [11]). Define-se a $\sigma$-álgebra de Borel, ou boreliana $\mathbb{X}$ de $X$ como sendo a $\sigma$-álgebra gerada pelos abertos de $X$. Assim $\mathbb{X}=\sigma(\tau)$. Se $(X, \mathbb{X})$ e $(Y, \mathbb{Y})$ são espaços mensuráveis com $\mathbb{X}$ e $\mathbb{Y}$ suas respectivas $\sigma$-álgebras de Borel tem-se que toda a função contínua entre $X$ e $Y$ é mensurável. Em particular todo homeomorfismo entre $X$ e $Y$ é um isomorfismo mensurável.

Define-se a $\sigma$-álgebra produto de dois espaços mensuráveis $(X, \mathbb{X})$ e $(Y, \mathbb{Y})$ como sendo a $\sigma$-álgebra gerada por conjuntos da forma $A \times B \operatorname{com} A \in \mathbb{X}$ e $B \in \mathbb{Y}$, e denota-se por $\mathbb{X} \otimes \mathbb{Y}$. Assim $\mathbb{X} \otimes \mathbb{Y}=\sigma(\{E \subset X \times Y \mid E=A \times B, A \in \mathbb{X}, B \in \mathbb{Y}\})$. Se $(X, \mathbb{X})(Y, \mathbb{Y})$ são espaços topológicos separáveis então a $\sigma$-álgebra de Borel do espaço topológico $X \times Y$ é igual a $\mathbb{X} \otimes \mathbb{Y}[4]$.

Uma função $\mu: \mathbb{X} \rightarrow \overline{\mathbb{R}}^{+}$é chamada de medida sobre a $\sigma$-álgebra $\mathbb{X}$ se satisfaz as seguintes condições:

1. $\mu(\emptyset)=0$;

2. Se $\left(A_{n}\right)_{n \in \mathbb{N}}$ é sequência de elementos de $\mathbb{X}$ dois a dois disjuntos então $\mu\left(\cup A_{n}\right)=$ $\sum \mu\left(A_{n}\right)$;

neste caso a tripla ordenada $(X, \mathbb{X}, \mu)$ é chamada de espaço de medida. Caso $\mu(X)=1$ então $\mu$ é chamada de medida de probabilidade e $(X, \mathbb{X}, \mu)$ é chamado de espaço de probabilidade.

Seja $X$ um espaço topológico e $\mu$ uma medida de Borel em $X$, i.e., uma medida definida na $\sigma$-álgebra de Borel de $X$, e seja $E$ um subconjunto mensurável de X, $\mu$ é chamada externamente regular em $E$ se

$$
\mu(E)=\inf \{\mu(U) \mid E \subset U, U \text { aberto }\}
$$


e é chamada internamente regular em $E$ se

$$
\mu(E)=\sup \{\mu(K) \mid K \subset E, K \text { compacto }\}
$$

Se $\mu$ é uma medida externamente e internamente regular em todos os borelianos de $X$ então $\mu$ é uma medida regular. Pelo resultado 7.8, p. 217 de [4], tem-se que toda medida de probabilidade boreliana definida em um espaço topológico métrico compacto $X$ é regular.

Uma função característica, ou indicadora, de um conjunto mensurável $A \subset X$, denotada por $\chi_{A}$ é tal que $\chi_{A}(x)=1, \forall x \in A$ e $\chi_{A}(x)=0, \forall x \in X \backslash A . \chi_{A}: X \rightarrow \overline{\mathbb{R}}^{+}$é obviamente mensurável. Uma função simples $\varphi: X \rightarrow \overline{\mathbb{R}}^{+}$é qualquer função mensurável que pode ser escrita como combinação linear finita de funções características, assim existem $A_{i}, 1 \leq i \leq n$ conjuntos mensuráveis e $a_{i}, 1 \leq i \leq n$ constantes reais tais que

$$
\varphi(x)=\sum_{i=1}^{n} a_{i} \chi_{A_{i}} .
$$

Define-se a integral da função simples $\varphi$ em relação a medida $\mu$ como

$$
\int \varphi d \mu=\sum_{i=1}^{n} a_{i} \mu\left(A_{i}\right)
$$

Para uma função mensurável qualquer $\psi: X \rightarrow \overline{\mathbb{R}}^{+}$define-se a integral de $\psi$ com respeito a medida $\mu$ como

$$
\int \psi d \mu=\sup \left\{\int \varphi d \mu \mid \varphi \text { é função simples e } \varphi \leq \psi\right\} \text {. }
$$

Define-se $L^{+}(X)=\left\{\psi: X \rightarrow \overline{\mathbb{R}}^{+} \mid \psi\right.$ é mensurável $\}$. Segue o enunciado do Teorema da Convergência Monótona, TCM.

Teorema 2.1. Teorema da Convergência Monótona. Se $\left(f_{n}\right)_{n \in \mathbb{N}}$ é uma sequência em $L^{+}(X)$ tal que $f_{j} \leq f_{j+1}, \forall j \in \mathbb{N}$, e $f(x)=\lim _{n \rightarrow \infty} f_{n}(x), \forall x \in X$ então $\int f=\lim _{n \rightarrow \infty} \int f_{n}$.

Dada uma função mensurável $\psi: X \rightarrow \overline{\mathbb{R}}^{+}$, é sempre possível encontrar uma sequência crescente de funções simples $\left(\varphi_{n}\right)_{n \in \mathbb{N}}$ tais que $\varphi_{n} \rightarrow \psi$ pontualmente, i.e., $\lim _{n \rightarrow \infty} \varphi_{n}(x) \rightarrow$ $\psi(x), \forall x \in X$, além disso se $\psi$ for limitada, pode-se supor que esta convergência é uniforme [1]. Aplicando este resultado conjuntamente com o TCM tem-se $\int \psi d \mu=\lim _{n \rightarrow \infty} \int \varphi_{n} d \mu$.

Se diz que uma propriedade vale quase sempre, q.s., ou em quase todo ponto, q.t.p., quando esta é válida no complementar de um conjunto de medida nula. Assim seja $(X, \mathbb{X}, \mu)$ um espaço de medida e $p$ uma proposição sobre os pontos de $X$. $p$ é válida para q.t.p. $x \in X$ se $\exists N \in \mathbb{X}$ tal que $\mu(N)=0$ e $p(x)$ é verdadeira para todo $x \in X \backslash N$. Quando uma igualdade é válida quase sempre pode-se dizer que é uma igualdade mod 0 , assim sejam $A$ e $B$ dois conjuntos mensuráveis de tal sorte que existe um subconjunto mensurável $F$ de medida total tal que $F \cap A=F \cap B$ nestas condições se diz que $A=B \bmod 0$. 
Seja $\psi: X \rightarrow \mathbb{R}$ uma função mensurável. Define-se $\psi^{+}=(|\psi|+\psi) / 2$ e $\psi^{-}=(|\psi|-\psi) / 2$ as partes positivas e negativas de $\psi$. Note que $\psi^{+}$e $\psi^{-}$são positivas e mensuráveis, caso $\int \psi^{+} d \mu, \int \psi^{-} d \mu<\infty$ então se diz que $\psi$ é integrável . Neste caso define-se sua integral, $\int \psi d \mu$, como $\int \psi d \mu=\int \psi^{+} d \mu-\int \psi^{-} d \mu$. O conjunto de todas as funções integráveis em um espaço de medida $(X, \mathbb{X}, \mu)$ é denotado $L_{\mu}^{1}(X)$. A seguir o Teorema da Convergência Dominada, TCD, que é uma generalização do TCM, é enunciado [4].

Teorema 2.2. Teorema da Convergência Dominada : Seja $\left(f_{n}\right)_{n \in \mathbb{N}}$ uma sequência de funções em $L^{1}$ tal que $f_{n}(x) \rightarrow f(x)$ q.s. e existe função não negativa $g \in L^{1}$ tal que $\left|f_{n}\right| \leq g$ q.s. para todo n. Então $f \in L^{1}$ e $\int f=\lim _{n \rightarrow \infty} \int f_{n}$.

Sejam $\mu$ e $\nu$ duas medidas definidas sobre o mesmo espaço mensurável $(X, \mathbb{X})$, se diz que $\mu$ é absolutamente contínua em relação a $\nu$ caso $\mu$ "mora" em $\nu$, i.e., $\forall A \in \mathbb{X}$ tal que $\nu(A)=0 \Rightarrow \mu(A)=0$. Representa-se a relação de continuidade aboluta pelo símbolo $\ll$, no caso, $\mu \ll \nu$. É uma consequência do teorema de Radon-Nikodym que caso $\nu$ e $\mu$ sejam medidas $\sigma$-finitas com $\mu \ll \nu$ então existe uma função $f \in L(X, \mathbb{X}, \nu)$ tal que $\int g d \mu=\int g f d \nu$ para toda função $g \nu$ integrável [4] a função $f$ é chamada de derivada de Radon-Nikodym de $\nu$ com respeito a $\mu$.

Um espaço polonês é um espaço topológico separável e completamente metrizável com $\sigma$-álgebra gerada pelos conjuntos abertos, i.e., é um espaço topológico homeomorfo a um espaço métrico completo que tem subconjunto enumerável e denso e é dotado da $\sigma$-álgebra de Borel. Um espaço standart é um espaço topológico isomorfo a um subconjunto mensurável de um espaço polonês. Espaços standart e espaços poloneses são de interesse especial para este trabalho. Seguem alguns resultados referentes a estes espaços

Proposição 2.3. Dois espaços standart $M_{1}$ e $M_{2}$ são Borel isomorfos sse tem a mesma cardinalidade [8].

Proposição 2.4. Seja $M$ um espaço standart, e $A$ um subconjunto de $M$. A é standart sse A é um subconjunto mensurável de $M$ [12].

Proposição 2.5. O produto de dois espaços standart ( respectivamente poloneses) $(M, \mathcal{B}) e$ $\left(M^{\prime}, \mathcal{B}^{\prime}\right)$ é espaço standart (respectivamente polonês) [12].

Aqui termina a breve revisão dos conceitos fundamentais de Teoria da Medida que são utilizados no decorrer da dissertação. Esta revisão foi baseada nas referências [1] [4]. Como referência de topologia recomenda-se [11] [13].

\subsection{Partições}

Uma partição em um espaço de medida, como o nome já indica é uma forma de particionar este espaço em conjuntos mensuráveis. Exemplos incluem o conjunto das retas horizontais no $\mathbb{R}^{2}$, a partição trivial que é todo o conjunto, ou a partição discreta em que todo elemento do espaço é um elemento da partição. Uma desintegração de uma probabilidade em relação 
a uma partição é associar a cada elemento da partição uma probabilidade de tal sorte que $(i)$ suporte da probabilidade associada esteja no elemento da partição e (ii) a medida original possa ser obtida por um processo de integração das probabilidades. O teorema da desintegração de Rohklin fornece uma condição suficiente para que seja feita uma desintegração de uma probabilidade em relação a uma partição.

Seja $(X, \mathbb{X})$ um espaço mensurável. $\mathcal{P} \subset \mathbb{X}$ é dita uma partição sobre $X$ se os elementos de $\mathcal{P}$ são dois a dois disjuntos e a união de todos os elementos de $\mathcal{P}$ é $X$. Assim se $A, B \in \mathcal{P}$ $\operatorname{com} A \neq B$ então $A \cap B=\emptyset$ e $\bigcup_{A \in \mathcal{P}} A=X$.

Sejam $\mathcal{P}$ e $\mathcal{Q}$ duas partições sobre o espaço $(X, \mathbb{X})$. Pode-se definir a seguinte relação de ordem parcial não estrita $\preceq$, que age da seguinte maneira $\mathcal{P} \preceq \mathcal{Q}$ sse para cada $Q \in \mathcal{Q}$, $\exists P \in \mathcal{P}$ tal que $Q \subset P$. Os seguintes fatos são de fácil verificação $\mathcal{P} \preceq \mathcal{P}$; se $\mathcal{P} \preceq \mathcal{Q}$ e $\mathcal{Q} \preceq \mathcal{P}$ então $\mathcal{P}=\mathcal{Q}$; e finalmente se $\mathcal{P} \preceq \mathcal{Q}$ e $\mathcal{Q} \preceq \mathcal{Z}$ então $\mathcal{P} \preceq \mathcal{Z}$. Quando $\mathcal{P} \preceq \mathcal{Q}$, se diz que $\mathcal{Q}$ é mais refinada que $\mathcal{P}$.

Dadas duas partições $\mathcal{P}, \mathcal{Q}$ de $(X, \mathbb{X})$ define-se $\mathcal{P} \vee \mathcal{Q}$ como sendo a partição menos refinada que refina $\mathcal{P}$ e $\mathcal{Q}$. Assim se $\mathcal{Z}$ é tal que $\mathcal{P} \preceq \mathcal{Z}$ e $\mathcal{Q} \preceq \mathcal{Z}$ então $\mathcal{P} \vee \mathcal{Q} \preceq \mathcal{Z}$. É fácil ver que se $A \in \mathcal{P} \vee \mathcal{Q}$, $\log 0 A=P \cap Q$ para algum $P \in \mathcal{P}$ e algum $Q \in \mathcal{Q}$. Assim para partições $\mathcal{P}, \mathcal{Q}$ finitas tem-se que $\# \mathcal{P} \vee \mathcal{Q} \leq \# \mathcal{P} \times \# \mathcal{Q}$ e para partições enumeráveis $\# \mathcal{P} \vee \mathcal{Q}=\# \mathbb{N} \times \# \mathbb{N}=\# \mathbb{N}$. Onde para um conjunto $C$ o símbolo $\# C$ denota a cardinalidade de $C$. De forma equivalente, dada uma sequência $\left(\mathcal{P}_{n}\right)_{n \in \mathbb{N}}$ de partições de $(X, \mathbb{X})$ define-se $\bigvee_{n \in \mathbb{N}} \mathcal{P}_{n}$ como sendo a partição menos refinada que refina $\mathcal{P}_{n}, \forall n \in \mathbb{N}$. Assim se $\mathcal{Z}$ é tal que $\mathcal{P}_{n} \preceq \mathcal{Z}, \forall n \in \mathbb{N}$ então $\bigvee_{n \in \mathbb{N}} \mathcal{P}_{n} \preceq \mathcal{Z}$.

Dada uma partição $\mathcal{P}$ de $(X, \mathbb{X})$, o mesmo símbolo $\mathcal{P}$ será utilizado para denotar a função $\mathcal{P}: X \rightarrow \mathcal{P}$, que associa a cada $x \in X$ o elemento $\mathcal{P}(x) \in \mathcal{P}$ que contém $x$. Apesar da notação ser ambígua, o contexto torna claro qual dos dois significados possíveis estão sendo atribuídos ao símbolo. A função $\mathcal{P}$ induz em $X$ a relação de equivalência $x \sim y \Leftrightarrow \mathcal{P}(x)=\mathcal{P}(y)$, i.e., $x, y \in X$ são equivalentes sse eles estão contidos no mesmo elemento da partição $\mathcal{P}$.

Quando $(X, \mathbb{X})$ é um espaço de medida, com medida $\mu$ pode-se definir uma $\sigma$-álgebra e uma medida na partição $\mathcal{P}$. A $\sigma$-álgebra será induzida pela função $\mathcal{P}$, i.e., será o conjunto $\left\{E \subset \mathcal{P} \mid \mathcal{P}^{-1}(E) \in \mathbb{X}\right\}$, portanto $E \subset \mathcal{P}$ é mensurável sse $\mathcal{P}^{-1}(E) \subset X$ é mensurável. A medida $\hat{\mu}$ em $\mathcal{P}$ será o push-foward da medida $\mu$, i.e., $\hat{\mu}(E)=\mu\left(\mathcal{P}^{-1}(E)\right)$, para todo $E \subset \mathcal{P}$ mensurável.

Do ponto de vista do Teorema da Desintegração de Rokhlin será interessante trabalhar com partições cujos elementos possam ser obtidos por um processo de limite de elementos que possuem volume. A qualidade "ser obtido por um processo de limite" é definida a seguir.

Definição 2.6. $\mathcal{P}$ é dita partição mensurável se existem subconjuntos mensuráveis $E_{1}, E_{2}$, $\ldots, E_{n}, \ldots$ de $X$ tais que

$$
\mathcal{P}=\left\{E_{1}, X \backslash E_{1}\right\} \vee\left\{E_{2}, X \backslash E_{2}\right\} \vee \ldots \vee\left\{E_{n}, X \backslash E_{n}\right\} \vee \ldots \bmod 0
$$

Assim $\exists F_{0} \subset X, \mu\left(F_{0}\right)=1$ tal que $\forall P \in \mathcal{P}$ tem-se 


$$
P \cap F_{0}=E_{1}^{*} \cap E_{2}^{*} \cap \ldots \cap E_{n}^{*} \cap \ldots \cap F_{0}
$$

$\operatorname{com} E_{j}^{*}=E_{j}$ ou $E_{j}^{*}=X \backslash E_{j}, \forall j \geq 1$.

É consequência direta da definição que toda partição finita ou enumerável é uma partição mensurável. A seguinte equivalência fornece uma definição alternativa.

Proposição 2.7. $\mathcal{P}$ é mensurável sse existem partições finitas ou enumeráveis $\mathcal{P}_{1} \preceq \mathcal{P}_{2} \preceq$ $\ldots \preceq \mathcal{P}_{n} \preceq$... tais que $\mathcal{P}=\bigvee_{n=1}^{\infty} \mathcal{P} \bmod 0$.

Demonstração. Claramente a segunda definição implica na primeira. Por outro lado seja $\mathcal{P}=\bigvee_{n=1}^{\infty} \mathcal{P}_{n}$ e para cada $i \in \mathbb{N}$ seja $\left\{P_{i, j}\right\}_{j \in \mathbb{N}}$ enumeração dos elementos de $\mathcal{P}_{i}$. Dado $P \in \mathcal{P}$ nota-se que devido ao fato da sequência de partições ser não decrescente garante-se que para cada partição $\mathcal{P}_{i}, \exists ! P_{i, j(i)} \in \mathcal{P}_{i}$ tal que $P \subset P_{i, j(i)}$. Assim pode-se escrever

$$
P=\bigcap_{i \in \mathbb{N}} P_{i, j(i)}=\bigcap_{i \in \mathbb{N}} P_{i, j(i)} \cap \bigcap_{i, k \in \mathbb{N}, k \neq j(i)} X \backslash P_{i, k} \quad \bmod 0 .
$$

Após reenumeração dos $P_{i, j}$ pode se escrever

$$
P=P_{1}^{*} \cap P_{2}^{*} \cap \ldots \cap P_{n}^{*} \cap \ldots \bmod 0
$$

donde segue a equivalência.

Exemplo 2.8. A partição por singletons de $[0,1]$ é mensurável, i.e., $\mathcal{P}=\{\{x\} \mid x \in[0,1]\}$ é mensurável.

Demonstração. Define-se

$$
\mathcal{P}_{n}=\left\{\left[0, \frac{1}{2^{n}}\right),\left[\frac{1}{2^{n}}, \frac{2}{2^{n}}\right), \ldots,\left[\frac{2^{n}-1}{2^{n}}, 1\right]\right\}, \forall n \in \mathbb{N}
$$

$\operatorname{logo}\left(\mathcal{P}_{n}\right)_{n \in \mathbb{N}}$ é sequência crescente de partições finitas. Além disso $\mathcal{P}=\bigvee_{n=1}^{\infty} \mathcal{P}_{n}$ pois se $x \in[0,1]$, tem-se que $\{x\} \subset \bigcap_{n \in \mathbb{N}} \mathcal{P}_{n}(x)$ trivialmente, e suponha que exista $y \neq x$ tal que $y \in \bigcap_{n \in \mathbb{N}} \mathcal{P}_{n}(x)$, isto implica que a distância entre $x$ e $y$ é menor do que $\frac{1}{2^{i}}$ para todo $i \in \mathbb{N}$, absurdo.

Uma observação importante sobre o exemplo anterior é que apesar da partição ser mensurável, a $\sigma$-álgebra gerada por $\mathcal{P}, \sigma(\mathcal{P})$, não é enumeravelmente gerada, i.e., $\sigma(\mathcal{P}) \neq$ $\sigma\left(\left\{A_{1}, A_{2}, \ldots\right\}\right)$ para toda sequência $\left(A_{n}\right)_{n \in \mathbb{N}}$ segue a demonstração deste fato. 
Demonstração. Supõe-se que exista sequência de subconjuntos de $[0,1],\left(A_{n}\right)_{n \in \mathbb{N}}$, tal que $\sigma(\mathcal{P})=\sigma\left(\left\{A_{1}, A_{2}, \ldots\right\}\right)$. Neste caso nota-se que todo $A_{n}$ pertence a $\sigma(\mathcal{P})$, e que

$$
\sigma(\mathcal{P})=\left\{E \subset[0,1] \mid E \text { é enumerável ou } E^{c} \text { é enumerável }\right\} .
$$

Com $E^{c}$ sendo o complementar do conjunto $E$ em relação ao conjunto [0,1]. Portanto cada $A_{n}$ é enumerável ou $A_{n}^{c}$ é enumerável. Para cada $n$ tal que $A_{n}$ satisfaz a última condição troca-se $A_{n}$ por $A_{n}^{\prime}=A_{n}^{c}$. Nota-se que apesar da troca a $\sigma$-álgebra gerada pela sequência de conjuntos continua a mesma pois

$$
\sigma\left(\left\{A_{1}, A_{2}, \ldots\right\}\right)=\sigma\left(\left\{A_{1}^{*}, A_{2}^{*}, \ldots\right\}\right)
$$

Assim sem perda de generalidade pode-se supor que todos os $A_{n}$ são enumeráveis. Define-se $A=\bigcup_{n \in \mathbb{N}} A_{n} \mathrm{e}$

$$
\mathcal{F}=\left\{F \subset[0,1] \mid F \subset A \text { ou } F=G \cup A^{c}, \text { para algum } G \subset A\right\}
$$

é simples verificar que $\mathcal{F}$ é uma $\sigma$-álgebra. E além disso $A_{i} \subset \mathcal{F}, \forall i \in \mathbb{N}$, e portanto $\mathcal{F} \supset \sigma\left(\left\{A_{1}, A_{2}, \ldots\right\}\right)$. Mas $A^{c}$ é obviamente não vazio, assim escolhe-se $x \in A^{c}$. Pela definição de $\mathcal{F}$ tem-se que $\{x\} \notin \mathcal{F}$. Assim

$$
\sigma(\mathcal{P}) \neq \sigma\left(\left\{A_{1}, A_{2}, \ldots\right\}\right)
$$

a contradição prova que $\sigma(\mathcal{P})$ não é enumeravelmente gerado.

O próximo exemplo pode ser utilizado para construir partições não mensuráveis.

Exemplo 2.9. Seja $f: X \rightarrow X$ transformação mensurável tal que $(f, \mu)$ é ergódica. Seja $\mathcal{P}$ partição de $X$ cujos elementos são órbitas de $f$. Se $f$ não possui nenhuma órbita com medida total, então $\mathcal{P}$ não é mensurável.

Demonstração. Supõe-se que exista sequência $\left\{\mathcal{P}_{n}\right\}_{n \in \mathbb{N}}$ de partições enumeráveis não decrescentes tal que $\mathcal{P}=\vee_{n=1}^{\infty} \mathcal{P}_{n} \bmod 0$. Todo elemento $P \in \mathcal{P}_{n}$ é invariante pela ação de $f$, este fato juntamente com a ergodicidade de $(f, \mu)$ implica $\mu(P)=0$ ou 1 . Segue que quase toda órbita de $f$ está contida em algum elemento $P_{n}$ da partição $\mathcal{P}_{n}$. Denote $P=\cap_{n=1}^{\infty} P_{n}$. Então $\mu(P)=1$ e $P$ é uma órbita de $f$, a conclusão segue pela contra positiva.

O próximo exemplo é uma generalização do exemplo 2.8 .

Exemplo 2.10. Seja $X=Y \times Z$, com $Y, Z$ espaços métricos compactos e $\mathcal{Q}$ uma partição de $X$ em linhas horizontais $Y \times\{z\}, z \in Z$. Então $\mathcal{Q}$ é partição mensurável de $X$. 
Demonstração. $Z$ é métrico e compacto, donde é separável, seja $\left\{x_{j}\right\}_{j \in \mathbb{N}}$ conjunto enumerável e denso de $Z$. Define-se $I(x, i, n)=\left\{z \in Z \mid(i-1) / 2^{n} \leq d(z, x)<i / 2^{n}\right\}$, i.e., $I(x, i, n)$ é o setor circular em $Z$ centrado em $x \in Z$ com raio interno $(i-1) / 2^{n}$ e raio externo $i / 2^{n}$. Define-se as partições

$$
\mathcal{P}_{n, m}=\left\{Y \times I\left(x_{n}, i, m\right) \mid i=1,2, \ldots, 2^{m}\right\}
$$

e define-se indutivamente a sequêcia de partições $\left(\mathcal{Q}_{n}\right)_{n \in \mathbb{N}}$.

$$
\begin{gathered}
\mathcal{Q}_{1}=\left\{Y \times I\left(x_{0}, i, 0\right) \mid i=1\right\}=\mathcal{P}_{0,0} \\
\mathcal{Q}_{2}=\left\{Y \times I\left(x_{0}, i, 1\right) \mid i=1,2\right\} \vee\left\{Y \times I\left(x_{1}, i, 0\right) \mid i=1\right\}=\mathcal{P}_{0,1} \vee \mathcal{P}_{1,0} \\
\mathcal{Q}_{3}=\left\{Y \times I\left(x_{0}, i, 2\right) \mid i=1,2,3,4\right\} \vee\left\{Y \times I\left(x_{1}, i, 1\right) \mid i=1,2\right\} \vee\left(Y \times I\left(x_{2}, 1,0\right)\right)= \\
\mathcal{P}_{0,2} \vee \mathcal{P}_{1,1} \vee \mathcal{P}_{2,0}
\end{gathered}
$$

Assim $\mathcal{Q}_{n}=\bigvee_{i=0}^{n} \mathcal{P}_{i, n-i}$. Repare que para cada $n$ a partição $\mathcal{Q}_{n}$ é finita, e tem-se que

$$
\mathcal{Q}=\bigvee_{n \in \mathbb{N}} \mathcal{Q}_{n}
$$

donde segue que $\mathcal{Q}$ é partição mensurável.

Antes de terminar esta seção mais alguns resultados sobre espaços standart são apresentados.

Proposição 2.11. O quociente $X / \mathcal{P}$ de um espaço standart $X$ por uma partição mensurável $\mathcal{P}$ é um espaço standart ([12], [15]).

Definição 2.12. O gráfico de uma relação de equivalência em um espaço $X$ é o subconjunto $X \times X$ de pares $(x, y)$, onde $x$ é equivalente a $y$.

Proposição 2.13. Seja $\mathcal{P}$ uma partição mensurável em um espaço standart $M$. O gráfico $R$ da partição $\mathcal{P}$ (considerado como uma relação de equivalência) é um subspaço mensurável de $M \times M[16]$.

\subsection{O Teorema da Desintegração de Rokhlin}

Definição 2.14. Seja $(X, \mathbb{X}, \mu)$ um espaço de probabilidade e $\mathcal{P}$ uma partição sobre $X$. Um sistema de medidas condicionais de $\mu$ em relação a $\mathcal{P}$, também chamado de desintegração de $\mu$ em relação a $\mathcal{P}$, é uma família $\left(\mu_{P}\right)_{P \in \mathcal{P}}$ de medidas de probabilidade em $X$ tal que

1. $\mu_{P}(P)=1$, $\hat{\mu} . q . t . P \in \mathcal{P}$ 
2. Dada qualquer função integrável $\psi: X \rightarrow \mathbb{R}$, a função $\mathcal{P} \ni P \rightarrow \int \psi d \mu_{P}$ é mensurável $\mathrm{e} \int \psi d \mu=\int\left(\int \psi d \mu_{P}\right) d \hat{\mu}(P)$

O teorema da desintegração de Rokhlin garante que quando $X$ é um espaço métrico compacto, e $\mathcal{P}$ é uma partição mensurável sobre $X$, então para toda medida de probabilidade em $X$ existe uma desintegração em relação a partição $\mathcal{P}$. Seja $\mu$ probabilidade em $X$. A ideia básica comumente utilizada $[17]^{2},[14]$ para construir a família de medidas que desintegra $\mu$ é a seguinte: Primeiramente escreve-se $\mathcal{P}=\bigvee \mathcal{P}_{n}$, $\operatorname{com} \mathcal{P}_{n}$ sequência decrescente de partições. Dado um elemento $P \in \mathcal{P}$ tem-se que $P=\bigcap \mathcal{P}_{n}(P)$, a medida $\mu_{P}$, é "definida" da seguinte forma

$$
\mu_{P}(A)=\lim _{n \rightarrow \infty} \frac{1}{\mu\left(\mathcal{P}_{n}(P)\right)} \int_{\mathcal{P}_{n}(P)} \chi_{A} d \mu
$$

para todo conjunto $A$ mensurável. Assim a probabilidade $\mu_{P}$ é obtida ao se tomar uma sequência de médias sobre conjuntos cada vez menores.

Para esta dissertação não é utilizada esta técnica. Apesar disto, o conceito de média é apresentado, e a partir deste conceito é obtida a demonstração, mas o passo de tirar sucessivas médias é substituído por uma aplicação inteligente da derivada de Radon-Nikodym. Esta demonstração foi fortemente baseada na demonstração apresentada no livro de Einsiedler e Ward [6].

Teorema 2.15. [6] Seja $(X, \mathbb{B}, \mu)$ espaço de probabilidade, seja $\mathbb{A} \subset \mathbb{B}$ uma $\sigma$-álgebra. Então existe uma aplicação

$$
E(\cdot \mid \mathbb{A}): L^{1}(X, \mathbb{B}, \mu) \rightarrow L^{1}(X, \mathbb{A}, \mu)
$$

denominada de esperança condicional que satisfaz as seguintes propriedades:

(1) Para $f \in L^{1}(X, \mathbb{B}, \mu)$, a imagem de $f, E(f \mid \mathbb{A})$, é caracterizada pelas propriedades:

- $E(f \mid \mathbb{A})$ é $\mathbb{A}$-mensurável;

- Para todo conjunto $A \in \mathbb{A}, \int_{A} E(f \mid \mathbb{A}) d \mu=\int_{A} f d \mu$.

(2) $E(\cdot \mid \mathbb{A})$ é positivo, i.e. $E(f \mid \mathbb{A}) \geq 0$ quase sempre se $f \in L^{1}(X, \mathbb{B}, \mu)$ satisfaz $f \geq 0$;

(3) Se $f \in L^{1}(X, \mathbb{A}, \mu)$ então $E(f \mid \mathbb{A})=f$ quase sempre.

Demonstração. (1) Supõe-se que $f \geq 0$. Então a medida definida por

$$
\mu_{f}(B)=\int_{B} f d \mu
$$

\footnotetext{
${ }^{2}$ Deve-se ter algum cuidado com esta publicação online, uma vez que a demonstração de três linhas da proposição 7 parece estar incorreta ou incompleta.
} 
é finita e absolutamente contínua a $\mu \mathrm{em}(X, \mathbb{B})$. Segue que $\left.\mu_{f}\right|_{\mathbb{A}}$ é absolutamente contínua com respeito a $\left.\mu\right|_{\mathbb{A}}$, assim existe uma derivada de Radon-Nikodym $g$ em $L^{1}(X, \mathbb{A}, \mu)$ tal que

$$
\mu_{f}(A)=\int_{A} g d \mu
$$

portanto

$$
\int_{A} f d \mu=\int_{A} g d \mu
$$

para todo conjunto $A \in \mathbb{A}$. Fazendo $g=E(f \mid \mathbb{A})$ tem-se a existência, resta provar que $g$ satisfaz as demais propriedades.

(3) Prova-se a unicidade da esperança condicional no complementar de conjuntos de medida nula. De fato se $g_{1}$ e $g_{2}$ satisfazem ambas as propriedades então

$$
A=\left\{x \mid g_{1}(x)<g_{2}(x)\right\} \in \mathbb{A}
$$

tem-se que

$$
\int_{A} g_{1} d \mu=\int_{A} f d \mu=\int_{A} g_{2} d \mu
$$

e $\log 0 \mu(A)=0$, de modo similar $\mu\left(\left\{x \mid g_{1}(x)>g_{2}(x)\right\}\right)=0$. Assim $g_{1}=g_{2}$ quase sempre. Uma vez provada a unicidade prova-se a linearidade facilmente.

(2) Para a positividade seja $f$ função não negativa em $L^{1}(X, \mathbb{B}, \mu)$ e seja

$$
A=\{x \in X \mid E(f \mid \mathbb{A}) \leq 0\}
$$

então

$$
0 \leq \int_{A} f d \mu=\int_{A} E(f \mid \mathbb{A}) d \mu
$$

implica que $\mu(A)=0$.

Definição 2.16. Seja $(A, \mathbb{A})$ um espaço mensurável e seja $x \in A$ um ponto qualquer. Definese o átomo de $\mathbb{A}$ em $x,[x]$, como sendo o conjunto

$$
[x]=\bigcap_{x \in A \in \mathbb{A}} A
$$

Observa-se que como a interseção não é necessariamente enumerável $[x]$ pode não ser um conjunto mensurável. A proposição a seguir mostra que o átomo de qualquer ponto em uma $\sigma$ álgebra gerada por uma partição é o próprio elemento da partição que contém o ponto.

Proposição 2.17. Seja $X$ um conjunto, $\mathcal{P}$ uma partição deste conjunto e seja $x \in X$ um elemento qualquer. Tem-se que não existe $B \in \sigma(\mathcal{P})$ tal que $x \in B, B \subset \mathcal{P}(x)$ e $B \neq \mathcal{P}(x)$. Em outras palavras o átomo de $x$ em $\sigma(\mathcal{P})$ é $\mathcal{P}(x)$. 
Demonstração. Considera-se a partição $\mathcal{P} \backslash\{\mathcal{P}(x)\}$ de $X \backslash \mathcal{P}(x)$. Seja $\mathbb{A}=\sigma(\mathcal{P} \backslash\{\mathcal{P}(x)\}) \sigma$ álgebra em $X \backslash \mathcal{P}(x)$. E define-se

$$
\mathbb{A}^{\prime}=\{A \subset X \mid A \in \mathbb{A} \text { ou } A=E \cup \mathcal{P}(x), \text { com } \mathrm{E} \in \mathbb{A}\}
$$

é fácil verificar que $\mathbb{A}^{\prime}$ é $\sigma$-álgebra em $X$, além disso segue da definição que $\mathbb{A}^{\prime} \supset \sigma(\mathcal{P})$. Mas pela definição de $\mathbb{A}^{\prime}$ tem-se que $\mathcal{P}(x)$ é átomo de $\mathbb{A}^{\prime}$, como $\mathbb{A}^{\prime} \supset \sigma(\mathcal{P})$ e $\mathcal{P}(x) \in \sigma(\mathcal{P})$ então $\mathcal{P}(x)$ é átomo de $x$ em $\sigma(\mathcal{P})$. Portanto não existe $B \in \sigma(\mathcal{P})$ tal que $x \in B, B \subset \mathcal{P}(x)$ e $B \neq \mathcal{P}(x)$, como queria demonstrar.

A importância de que os elementos da partição sejam átomos é de que qualquer função que seja $\sigma(\mathcal{P})$-mensurável é constante em cada elemento da partição. Este fato é utilizado na próxima demonstração.

A seguir demonstra-se o teorema da desintegração de Rokhlin. O teorema é apresentado aqui baseado na demonstração presente no livro [6]. Neste livro a desintegração é realizada em relação aos átomos de uma sub- $\sigma$-álgebra enumeravelmente gerada. Conforme mostrado nos comentários após o exemplo 2.8 a $\sigma$-álgebra gerada por uma partição mensurável não é necessariamente enumeravelmente gerada. Assim para obter a desintegração em relação aos átomos de uma partição mensurável é necessário estender o teorema. Felizmente esta extensão é trivial.

Teorema 2.18. [6] (Teorema da Desintegração de Rohklin) Seja X um espaço métrico compacto e seja $(X, \mathbb{B}, \mu)$ um espaço de probabilidade boreliana. $\mathbb{A} \subset \mathbb{B}$ é uma $\sigma$-álgebra de $\mathbb{B}$ e $\mathcal{P}$ é partição mensurável de $X$. Existe um conjunto de medida total $X^{\prime} \subset X$ e um sistema de medidas $\left\{\mu_{x} \mid x \in X^{\prime}\right\}$ sobre $X$, denominado sistema de medidas condicionais com as seguintes propriedades

(1) $\mu_{x}$ é medida em $X$ com

$$
E(f \mid \mathbb{A})(x)=\int f(y) d \mu_{x}(y)
$$

quase sempre para toda $f$ integrável. Em outras palavras, para toda $f$ integrável, $\int f(y) d \mu_{x}(y)$ existe para quase todo $x$ e a aplicação

$$
x \mapsto \int f(y) d \mu_{x}(y)
$$

é $\mathbb{A}$-mensurável e além disso

$$
\int_{A} \int f(y) d \mu_{x}(y) d \mu(x)=\int_{A} f d \mu
$$

para todo $A \in \mathbb{A}$. 
(2) Se $\mathcal{P}$ é partição mensurável de $X$, então

$$
\mu_{\mathcal{P}(x)}(\mathcal{P}(x))=1, \forall x \in X^{\prime}
$$

$\operatorname{com} \mu_{\mathcal{P}(x)}=\mu_{x}, \forall x \in X^{\prime}$.

(3) Propriedade (1) determina $\mu_{x}$ unicamente para quase todo $x \in X$.

Demonstração. (1) Para a existência, seja

$$
\mathscr{F}=\left\{f_{0} \equiv 1, f_{1}, f_{2}, \ldots\right\} \subset C(X)
$$

um espaço vetorial sobre $\mathbb{Q}$ que é denso em $C^{0}(X, \mathbb{R})$. Escolha de tal $\mathscr{F}$ é possível pois $C^{0}(X, \mathbb{R})$ admite um subconjunto denso e enumerável, e o spam deste conjunto sobre o corpo dos racionais é um espaço vetorial enumerável. Para cada $i \geq 1$, escolha uma função $\mathbb{A}$-mensurável e $\mu$-integrável $g_{i} \operatorname{com} g_{i}=E\left(f_{i} \mid \mathbb{A}\right)$. Define-se $g_{0}$ como sendo a função constante 1. Então

- $g_{i} \geq 0$ quase sempre se $f_{i} \geq 0$;

- $\left|g_{i}(x)\right| \leq\left\|f_{i}\right\|_{\infty}$ quase sempre;

- se $f_{i}=\alpha f_{j}+\beta f_{k} \operatorname{com} \alpha, \beta \in \mathbb{Q}$, então $g_{i}(x)=\alpha g_{j}(x)+\beta g_{k}(x)$ para quase todo $\mathrm{x}$.

Seja $N \in \mathbb{A}$ a união de todos os conjuntos nulos no complementar donde as propriedades acima são válidas; como esta união é enumerável, $N$ é um conjunto nulo para $\mu$.

Para $x \notin N$, defina $\Delta_{x}\left(f_{i}\right)$ como sendo $g_{i}(x)$. Então pelas propriedades acima $\Delta_{x}$ é uma aplicação $\mathbb{Q}$-linear de $\mathcal{F}$ em $\mathbb{R}$ com $\left\|\Delta_{x}\right\| \leq 1$. Segue que se $\Delta_{x}$ se estende de forma única a um funcional linear continuo e positivo

$$
\Delta_{x}: C(X) \rightarrow \mathbb{R}
$$

Pelo teorema da representação de Riesz [6] existe uma medida $\mu_{x}$ em $X$ caracterizada pela propriedade

$$
\Delta_{x}(f)=\int f d \mu_{x}
$$

para toda $f \in C(X)$, além disso $\Delta_{x}(1)=1$ pois $E(1 \mid \mathbb{A})=1$. Portanto $\mu_{x}$ é medida de probabilidade. Pela escolha de conjunto $\mathscr{F}$, para toda $f \in C(X)$ existe sequência $\left(f_{n i}\right)$ con $f_{n i} \rightarrow f$ uniformemente. Já foi estabelecido que

$$
x \mapsto \int f_{n i} d \mu_{x}
$$

é $\mathbb{A}$-mensurável uma vez que é equivalente a $x \mapsto E\left(f_{n i} \mid \mathbb{A}\right)(x)$ e que

$$
\int_{A} \int f_{n i} d \mu_{x} d \mu(x)=\int_{A} f_{n i} d \mu
$$


para todo conjunto $A \in \mathbb{A}$ pois $\int_{A} E\left(f_{n i} \mid \mathbb{A}\right)(x) d \mu(x)=\int_{A} f_{n i} d \mu$. Assim pelo teorema de convergência dominada (lembra-se que pode-se supor convergência uniforme) a função

$$
x \mapsto \int f d \mu_{x}
$$

é $\mathbb{A}$-mensurável pois é o limite de uma sequência de funções reais mensuráveis $x \mapsto \int f_{n i} d \mu_{x}$. Além disso por argumento similar segue que

$$
\int_{A} \int f d \mu_{x} d \mu(x)=\int_{A} f d \mu
$$

para todo $A \in \mathbb{A}$. Assim prova-se (1) para toda a $f \in C(X)$, i.e., toda $\mathrm{f}$ contínua. A seguir estende-se este resultado para toda a $f$ integrável.

Seja $\mathfrak{C}$ a classe de funções que satisfazem (1), segue que $C(X) \subset \mathfrak{C}$.

Se A é um conjunto aberto de $X$ então $\chi_{A}$, função característica do conjunto $A$, pertence a $\mathfrak{C}$. Para mostrar este fato define-se a função $f: X \rightarrow \mathbb{R}$ por $f(x)=d(x, X \backslash A)$, sendo $d$ a métrica de $\mathrm{X}$. Note que $f$ é contínua e portanto limitada. Considera-se a sequência de funções contínuas $f_{n}=\sqrt[n]{f}, \forall n \in \mathbb{N}$, cada $f_{n}$ é contínua e $f_{n} \rightarrow \chi_{A}$ pontualmente além disso $\max \left|f_{n}\right| \leq \max \{\max |f|, 1\}$. Assim pelo teorema da convergência dominada, TCD, segue que $\chi_{A}$ satisfaz as condições do lema.

Seja $\mathcal{F} \subset \mathfrak{C}$ a família de todas as funções características de conjuntos mensuráveis que satisfazem a conclusão da proposição. Nota-se que se $\left(\chi_{A_{n}}\right)_{n \in \mathbb{N}}$ é sequência de elementos em $\mathcal{F}, \operatorname{logo} \chi_{\cup A_{n}}=\lim _{n} \sup \left\{\chi_{A_{1}}, \chi_{A_{2}}, \ldots, \chi_{A_{n}}\right\}$ também pertence a $\mathcal{F}$ pelo TCD. Também tem-se que se $\chi_{A} \in \mathcal{F}, \stackrel{n}{\operatorname{logo}} 1-\chi_{A} \in \mathcal{F}$ e portanto $\chi_{X \backslash A} \in \mathcal{F}$. Pelo parágrafo anterior tem-se que as funções características de todos os conjuntos abertos estão em $\mathcal{F}$. Assim a classe de conjuntos mensuráveis cujas funções características estão em $\mathcal{F}$ contém todos os abertos e é fechada por complementar e união enumerável, donde segue que é toda a $\sigma$-álgebra de $X$.

Tem-se que todas as funções características de conjuntos mensuráveis estão em $\mathfrak{C}$, e que este conjunto é fechado por convergência pontual crescente de funções positivas pelo TCM. Donde segue que todas as funções $\mathbb{B}$-mensuráveis positivas estão em $\mathfrak{C}$. Finalmente dada uma função $f \mathbb{B}$-mensurável e integrável qualquer, pode-se escrever

$$
f=f^{+}-f^{-}
$$

com $f^{+}, f^{-}$não negativas, mensuráveis e integráveis. Então por (2.2)

$$
\int f^{+} d \mu_{x}, \int f^{-} d \mu_{x}<\infty
$$

quase sempre. Em particular, $f$ é $\mu_{x}$-integrável para quase todo $x$, donde é $\mu_{x}$-integrável, $\int f d \mu_{x}$ é função $\mathbb{A}$ mensurável de $x$. Assim 5.9 é verdadeira provando (1).

(2) Suponha agora que $\mathbb{A}=\sigma(\mathcal{P})$, i.e., $\mathbb{A}$ é gerada por uma partição mensurável. Então 
pelo item (3) do teorema 2.15 tem-se que

$$
E\left(\chi_{\mathcal{P}(x)} \mid \mathbb{A}\right)(x)=\chi_{\mathcal{P}(x)}(x)=\mu_{x}(\mathcal{P}(x))
$$

para quase todo $\mathrm{x}$. Logo

$$
\mu_{x}(\mathcal{P}(x))=1
$$

quase sempre. Relembra-se que o mapa

$$
X^{\prime} \ni x \mapsto \int f d \mu_{x}
$$

é $\mathbb{A}$-mensurável para todo $f \in C(X)$. Logo utilizando a mensurabilidade juntamente com o resultado 2.17 tem-se que

$$
\int f d \mu_{x}=\int f d \mu_{y}
$$

se $x, y \in X^{\prime}$ e $\mathcal{P}(x)=\mathcal{P}(y), \operatorname{logo} \mathcal{P}(x)=\mathcal{P}(y)$ implica que $\mu_{x}=\mu_{y}$. Assim pode-se identificar $\mu_{x} \operatorname{com} \mu_{\mathcal{P}(x)}$.

(3) Procede-se a prova da unicidade.

Supõe-se que $\left\{\rho_{x}\right\}$ e $\left\{\nu_{x}\right\}$ são famílias de medidas definidas para quase todo $x$ e que ambas satisfazem (1) para um conjunto enumerável e denso $\left\{f_{n}\right\}_{n \in \mathbb{N}}$ em $C^{0}(X, \mathbb{R})$. Então para cada $n \geq 1$ e para quase todo $\mathrm{x}$,

$$
\int f_{n} d \rho_{x}=E\left(f_{n} \mid \mathbb{A}\right)=\int f_{n} d \nu_{x}
$$

Assim existe um conjunto de medida $\mu$ nula $N$ com a propriedade de que (5.7) é válida para todo $n \geq 1$ e $x \notin N$. Pela convergência uniforme e o TCD tem-se que

$$
\int f d \rho_{x}=\int f d \nu_{x}
$$

para toda $f \in C^{0}(X, \mathbb{R})$ e $x \notin N$. Assim $\rho_{x}=\nu_{x}$ para $x \notin N$, o que mostra que as medidas condicionais são únicas.

\subsection{Sistemas Dinâmicos Uniformemente Hiperbólicos}

Um sistema dinâmico é uma dupla ordenada $(X, \mathcal{F})$, com $X$ um espaço dotado de alguma estrutura e uma família de mapas $\mathcal{F}=\left\{f_{t}: X \rightarrow X\right\}_{t \in A}$ que preservam a estrutura de $X$. O cojunto de índices $A$ é usualmente um grupo ou semigrupo. Assim caso $X$ seja um espaço topológico $\left\{f_{t}\right\}_{t \in A}$ é uma família de funções contínuas. O caso de interesse contemplado por este trabalho é quando $\mathcal{F}$ é uma família de bijeções cujas respectivas inversas também preservam a estrutura de $X$, neste caso diz-se que $\mathcal{F}$ é uma família de automorfismos de $X$. Assim caso $X$ seja um espaço de probabilidade os automorfismos de $X$ em $X$ correspondem 
a bijeções mensuráveis, com inversa mensurável e que preservem a medida (medida de um conjunto mensurável $B$ é igual a medida de $f(B)$ ).

O conjunto de índices $A$ usualmente é igual a $\mathbb{N}, \mathbb{Z}$ ou $\mathbb{R}$. Nos dois primeiros casos o sistema dinâmico é chamado de discreto. Caso $A=\mathbb{R}$ então o sistema dinâmico é chamado de contínuo. Em sistemas dinâmicos discretos tem-se que $f_{t}=\left(f_{1}\right)^{t}$.

Em sistemas dinâmicos, especialmente teoria ergódica, existe interesse em medidas que são invariantes pela dinâmica do sistema. Diz-se que uma medida $\mu$ é invariante por $f$ ou simplesmente $f$-invariante se $\mu(A)=\mu\left(f^{-1}(A)\right)$ para todo conjunto $A$ mensurável .

Este trabalho foca no caso em que $X$ é uma variedade $C^{2}$, e portanto dotada de uma métrica riemanniana $\|\| . f:. X \rightarrow X$ é um difeomorfismo $C^{2}$, e o sistema dinâmico é discreto.

Definição 2.19. Um sistema dinâmico $(M, f)$ é chamado de sistema dinâmico uniformemente hiperbólico se existe uma separação contínua do fibrado tangente $T M$, que é invariante sob ação do mapa diferencial $D f$, i.e.,

$$
T M=E^{s} \oplus E^{u}, \quad D f\left(E^{s}\right)=E^{s}, \quad D f\left(E^{u}\right)=E^{u}
$$

e tal que existem constantes $c$ e $\delta, c>0$ e $0<\delta<1$, tais que para todo $t \in \mathbb{Z}$,

$$
\begin{gathered}
\left|D f^{t}\right|_{E^{s}} \mid<c \delta^{t}, t \geq 0 \\
\left|D f^{-t}\right|_{E^{u}} \mid<c \delta^{t}, t \geq 0
\end{gathered}
$$

com $c, \delta$ independentes de $x \in M$.

É digno e nota que nem sempre possível encontrar uma métrica riemanniana adaptada em $X$ de forma que $c=1$.

Definição 2.20. Dado $x \in M$ definem-se os conjuntos estáveis $W^{s}(x)$ e instáveis $W^{u}(x)$ por

$$
\begin{gathered}
W^{s}(x)=\left\{y \in M \mid \lim _{n \rightarrow \infty} d\left(f^{n}(y), f^{n}(x)\right)=0\right\} \\
W^{u}(x)=\left\{y \in M \mid \lim _{n \rightarrow-\infty} d\left(f^{n}(y), f^{n}(x)\right)=0\right\}
\end{gathered}
$$

Dado $x \in M$ os conjuntos $W^{s}(x)$ e $W^{u}(x)$ definem classes de equivalência da forma $x \sim y \Leftrightarrow y \in W^{s, u}(x)$ [16]. Além disso os conjuntos globalmente instáveis e estáveis são folheações em $X$.

Definição 2.21. Define-se a taxa de expansão da variedade no ponto $x, J^{u}(x)$ relativo a uma métrica Riemanniana pela equação

$$
J^{u}(x)=\left|\operatorname{det}\left(\left.D f\right|_{E^{u}(x)}\right)\right|
$$

onde $\operatorname{det}\left(\left.D f\right|_{E^{u}(x)}\right)$ é o determinante da matriz $D f$ referente ao subespaço $E^{u}(x)$. 
Definição 2.22. Uma partição mensurável $\mathcal{P}$ de $M$ é dita subordinada a $W^{u}$ se para $\mu$ q.t.x, $\mathcal{P}(x)$ é um elemento de $\mathcal{P}$ contido em $W^{u}(x)$, i.e., $\mu$ q.t.x, $\mathcal{P}(x) \subset W^{u}(x)$, e para $\mu$ q.t. $x, \mathcal{P}(x)$ possui uma vizinhança aberta de $x$ na topologia de subvariedade de $W^{u}(x)$.

Definição 2.23. Seja $m_{x}$ a restrição da medida de Riemann em $W^{u}(x)$. Uma medida de probabilidade $f$-invariante $\mu$ tem medidas condicionais absolutamente contínuas em variedades instáveis se para toda partição mensurável $\mathcal{P}$ subordinada a $W^{u}$ tem-se $\mu_{\mathcal{P}(x)} \ll m_{\mathcal{P}(x)}$ M.q.t.x i.e., $\mu_{\mathcal{P}(x)}$ é absolutamente contínua em relação a medida de Riemann $m_{x}$ de $\mathcal{P}(x)$.

O próximo resultado relaciona desintegração de medidas de Riemann com medidas SRB, sendo portanto a motivação deste trabalho.

Teorema 2.24. [18] Seja $(M, f)$ um sistema uniformemente hiperbólico, com $f$ um difeomorfismo $C^{2}$. Então existe uma única medida de probabilidade boreliana $\mu$ em $M$ que é caracterizada por cada uma das condições equivalentes

(i) $\mu$ tem medidas condicionais absolutamente contínuas em variedades instáveis;

(ii) Se $h_{\mu}(f)$ é entropia métrica de f então

$$
h_{\mu}(f)=\int\left|\operatorname{det}\left(\left.D f\right|_{E^{u}}\right)\right| d \mu
$$

(iii) Existe um conjunto $V \subset M$ de medida de Lebesgue total tal que para cada função continua $\psi: M \rightarrow \mathbb{R}$ tem-se que $\forall x \in V$

$$
\frac{1}{n} \sum_{i=0}^{n-1} \psi\left(f^{i}(x)\right) \rightarrow \int \psi d \mu
$$

A medida $\mu$ é denominada medida SRB em homenagem aos pesquisadores pioneiros no assunto, Sinai, Ruelle e Bowen.

O próximo resultado é de interesse para o último capítulo desta dissertação.

Exemplo 2.25. Arnold's Cat Map. Seja $\mathbb{T}^{2}=\mathbb{R} / \mathbb{Z} \times \mathbb{R} / \mathbb{Z}$ o toro. Considere o sistema dinâmico $\left(\mathbb{T}^{2}, f\right) \operatorname{com} f: \mathbb{T}^{2} \rightarrow \mathbb{T}^{2}$ sendo a transformação linear dada por:

$$
f((x, y))=\left[\begin{array}{ll}
2 & 1 \\
1 & 1
\end{array}\right]\left[\begin{array}{l}
x \\
y
\end{array}\right]=\left[\begin{array}{ll}
1 & 1 \\
0 & 1
\end{array}\right]\left[\begin{array}{ll}
1 & 0 \\
1 & 1
\end{array}\right]\left[\begin{array}{l}
x \\
y
\end{array}\right] \bmod 1
$$

Observa-se que $\operatorname{det}(f)=1$ e logo é uma bijeção invariante pela medida de Lebesgue no toro, além disso os autovalores de $f$ são

$$
\lambda_{s}=\frac{3-\sqrt{5}}{2}<1 \quad \lambda_{u}=\frac{3+\sqrt{5}}{2}>1
$$


Define-se $E^{s}$ como sendo a reta em $\mathbb{R}^{2}$ definida pela equação $y=\lambda_{s} x$ e $E^{u}$ a reta definida pela equação $y=\lambda_{u} x$. Nota-se que $T M=E^{s} \oplus E^{u}, D f\left(E^{s}\right)=E^{s}$ e $D f\left(E^{u}\right)=E^{u}$. Além disso tem-se que

$$
\begin{aligned}
& \left|D f^{t}\right|_{E^{s}} \mid<\left(\lambda_{s}+\epsilon\right)^{t}, t \geq 0 \\
& \left|D f^{t}\right|_{E^{u}} \mid<\left(\lambda_{u}+\epsilon\right)^{t}, t \leq 0
\end{aligned}
$$

para $\varepsilon>0$ pequeno o suficiente, pois

$$
\begin{aligned}
& \left(D f^{t}\right) v=\lambda_{s}^{t} v, \forall v \in E^{s} . \\
& \left(D f^{t}\right) v=\lambda_{u}^{t} v, \forall v \in E^{u} .
\end{aligned}
$$

Para determinar $W^{s}$ e $W^{u}$ define-se $e_{s}$ e $e_{u}$ versores unitários na direção estável instável respectivamente, i.e., versores paralelos as retas $E^{s}$ e $E^{u}$ respectivamente. Assim $x \in \mathbb{T}^{2}$ pode ser escrito como

$$
x=\alpha_{s} e_{s}+\alpha_{u} e_{u}
$$

$\operatorname{com} \alpha_{s}, \alpha_{u} \in \mathbb{R}$. Assim

$$
\left|A^{n}\left(\alpha_{s} e_{s}+\alpha_{u} e_{u}\right)\right|=\left|\lambda_{s}^{n} \alpha_{s} e_{1}+\lambda_{u}^{n} \alpha_{u} e_{u}\right|=\sqrt{\left(\alpha_{s} \lambda_{s}^{n}\right)^{2}+\left(\alpha_{u} \lambda_{u}^{n}\right)^{2}}
$$

o que implica que

$$
\left|A^{n}\left(\alpha_{s} e_{s}+\alpha_{u} e_{u}\right)\right| \underset{n \rightarrow \infty}{\longrightarrow} 0 \Leftrightarrow \alpha_{u}=0
$$

logo $W^{s}(x)=x+E^{s}$, e de forma análoga $W^{u}(x)=x+E^{u}$.

Nota-se que

$$
J^{u}(x)=|\operatorname{det}(D f)|_{E^{u}(x)} \mid=\lambda_{u}
$$

Não é difícil ver que a partição do quadrado nas linhas finitas de inclinação $\delta_{u}$ é uma partição mensurável, e que sua desintegração em relação a esta partição é a própria medida de Borel unidimensional. Esta medida de Borel unidimensional corresponde a medida de Riemann em $W^{u}(x)$ para $\xi(x)$. Assim do item $(i)$ do teorema 2.24 segue que a medida de Borel bidimensional é a única medida $S R B$ do sistema.

\subsection{Sistemas Dinâmicos Não Uniformemente Hiperbólicos}

A seguinte definição foi particularizada.

Definição 2.26. [5] Dado um sistema dinâmico $(M, f)$, com $M$ variedade riemanniana e $f$ difeomorfismo $C^{2}$, define-se o expoente de Lyapunov de um ponto $x \in M$ e de um vetor 
$v \in T M$ por

$$
\lambda(x, v)=\lim _{n \rightarrow \infty} \frac{1}{n} \ln \left\|\left(D_{x} f\right)^{n}(v)\right\|
$$

para cada $x \in M$ a função $\lambda(x, \cdot)$ pode assumir no máximo um número finito de valores distintos $\lambda_{1}(x), \ldots, \lambda_{i}(x)$ que determinam os subespaços $V_{i}:=\left\{v \in T_{x} M \mid \lambda(x, v) \leq \lambda_{i}(x)\right\}$. Tem-se ainda que

$$
\{0\}=V_{0}^{+}(x) \subset \ldots \subset V_{i}(x)=T_{x} M
$$

Um sistema dinâmico $(M, f)$ em que para todos os pontos $x \in M$ e para todos os vetores $v \in T_{x} M \lambda(x, v) \neq 0$ será chamado de sistema hiperbólico não uniforme.

Para sistemas dinâmicos não uniformemente hiperbólicos as variedades instáveis $W^{u}$ satisfazem

$$
W^{u}(x)=\left\{y \in M \mid \limsup _{n \rightarrow \infty} \frac{1}{n} \ln \left(d\left(f^{-n}(x), f^{-n}(y)\right)\right)<0\right\} .
$$

As medidas SRB em sistemas dinâmicos não uniformemente hiperbólicos são aquelas que satisfazem o item (i) do teorema 2.24, i.e., são medidas cuja a desintegração em partições mensuráveis subordinadas as variedades instáveis é absolutamente contínua com respeito a medida de Lebesgue destas variedades. O seguinte teorema será necessário para o último capítulo desta dissertação.

Teorema 2.27. ([10] citado por [16]) Seja m uma medida SRB e seja $\mathcal{P}$ uma partição subordinada as variedades instáveis, seja $\rho$ a densidade da medida condicional $m_{\mathcal{P}(x)}$ com respeito a medida riemanniana de volume na variedade instável. Então para $\mu$-q.t. $x, \rho$ é uma função positiva estritamente positiva em $\mathcal{P}(x)$ satisfazendo

$$
\frac{\rho(y)}{\rho(x)}=\prod_{i=1}^{\infty} \frac{J^{u}\left(f^{-i} x\right)}{J^{u}\left(f^{-i} y\right)} .
$$




\section{Capítulo 3}

\section{Introdução a Teoria Não Comutativa de Inte- gração}

Neste capítulo é apresentada uma curta introdução a Teoria de Integração Não Comutativa de Alain Connes. O objetivo é estudar a relação entre medidas em um espaço com as medidas transversas definidas em um grupoide sobre este espaço (definições são apresentadas adiante). A última seção deste capítulo contém vários exemplos. Várias definições apresentadas são bastante abstratas, caso a leitura se torne enfadonha aconselha-se a ler o corpo do capítulo de forma paralela a seção de exemplos. Este capítulo é fortemente baseado no trabalho original de Connes, [3], e na posterior discussão de Kastler [7] sobre esta publicação. Também conta com algumas contribuições de Segert [16].

\subsection{Grupoides}

Um grupoide (em inglês grupoid) $G$ é uma categoria com inversão. Especificamente temse um conjunto $G^{(0)}$ que é o conjunto dos objetos tal que para cada $x, y \in G^{(0)}$ se associa um conjunto (possivelmente vazio) $G_{x}^{y}$ de morfismos $\gamma: x \rightarrow y, G$ é união disjunta de todos os $G_{x}^{y}, x, y \in G^{(0)}$. Além disso para cada par $\gamma: x \rightarrow y, \gamma^{\prime}: y \rightarrow z$, com $x, y, z \in G^{(0)}$, existe o produto $\gamma^{\prime} \gamma: x \rightarrow z$ com as seguintes propriedades:

(i) Associatividade: Se $\gamma: x \rightarrow y, \gamma^{\prime}: y \rightarrow z, \gamma^{\prime \prime}: z \rightarrow u, \operatorname{com} x, y, z, u \in G^{(0)}$ são morfismos em $G$, tem-se que $\gamma^{\prime \prime}\left(\gamma^{\prime} \gamma\right)=\left(\gamma^{\prime \prime} \gamma^{\prime}\right) \gamma$.

(ii) Elemento Neutro: Para cada $y \in G^{(0)}$, existe unidade $\mathbb{1}_{y}: y \rightarrow y$ com as propriedades $\mathbb{1}_{y} \gamma=\gamma$ e $\gamma^{\prime} \mathbb{1}_{y}=\gamma^{\prime}$ sempre que $\gamma: x \rightarrow y$ e $\gamma^{\prime}: y \rightarrow z, x, y, z, \in G^{(0)}$.

(iii) Elemento Inverso: Para cada $\gamma: x \rightarrow y$ existe $\gamma^{-1}: y \rightarrow x$ com a propriedade de que $\gamma^{-1} \gamma=\mathbb{1}_{x}$ e $\gamma \gamma^{-1}=\mathbb{1}_{y}$.

Do item $(i i)$ segue que $\mathbb{1}_{y}$ é único para cada $y \in G^{(0)}$, pois $\mathbb{1}_{y}^{\prime}=\mathbb{1}_{y}^{\prime} \mathbb{1}_{y}=\mathbb{1}_{y}$. Esta unicidade permite identificar cada $\mathbb{1}_{y}$ com $y$, e pode-se entender $G^{(0)}$ como um subconjunto de $G$. Do item (iii) tem-se que que $\gamma^{-1}$ é único.

Define-se a função $s: G \rightarrow G^{(0)}$ chamada de fonte (em inglês source) que associa a cada $\gamma: x \rightarrow y$, o domínio, i.e., $s(\gamma: x \rightarrow y)=x$. De forma análoga se define a função 
$r: G \rightarrow G^{(0)}$ denominada alvo (em inglês range ou target) que associa a cada $\gamma$ o contra domínio, i.e., $r(\gamma: x \rightarrow y)=y$. É denominado o conjunto de pares multiplicáveis $G^{(2)} \subset G \times G$ é definido pela propriedade $\left(\gamma, \gamma^{\prime}\right) \in G^{(2)}$ sse $s(\gamma)=r\left(\gamma^{\prime}\right)$.

Seja $A$ subconjunto de $G^{(0)}$, denota-se $G_{A}$ como sendo o grupoide obtido de $G$ ao restringir $G^{(0)}$ a $A$, i.e.,

$$
G_{A}=\{\gamma \in G \mid s(\gamma) \in A, r(\gamma) \in A\}
$$

Exemplo 3.1. Caso $G^{(0)}=\{x\}$, i.e., o conjunto de objetos é apenas um ponto, tem-se que $G$ é um grupo com elemento neutro $x$, e a operação de grupo composição de morfismos.

No contexto desta dissertação um grupoide $G$ deve ser entendido como um conjunto de flechas que conectam os objetos de $G^{(0)}$. Não se está particularmente interessado na estrutura das flechas. Isto se justifica pois como é visto adiante os objetos de $G^{(0)}$ são usualmente singletons e portanto desprovidos de estrutura interessante. O único interesse será em saber quando dois singletons estão relacionados por alguma flecha.

Um grupoide induz uma relação de equivalência $\sim \operatorname{em~} G^{(0)}$, em que dois objetos de $G^{(0)}$ são equivalentes se existe uma flecha que os conecta. Assim $x \sim y$ sse $\exists \gamma \in G$ tal que $s(\gamma)=x$ e $r(\gamma)=y$. É fácil verificar que $\sim$ é relação de equivalência [16].

Define-se a função

$$
\begin{aligned}
P: G & \rightarrow G^{(0)} \times G^{(0)} \\
\gamma & \mapsto(r(\gamma), s(\gamma))
\end{aligned}
$$

quando $G$ provém de uma relação de equivalência então diz-se que a imagem de $P$ é o gráfico da relação de equivalência.

Definição 3.2. $G$ é um grupoide principal se a função $P$ da equação (3.2) é injetiva.

A função $P$ ser injetiva implica que entre dois objetos quaisquer de $G^{(0)}$ existem no máximo duas flechas (em direções opostas) que conectam os dois elementos. Além disso a injetividade permite entender um grupoide principal $G$ como sendo um subconjunto de $G^{(0)} \times G^{(0)}$ associando-se uma flecha qualquer $\gamma: y \rightarrow x$ ao ponto $(x, y) \in G^{(0)} \times G^{(0)}$.

Existe uma correspondência bijetiva entre relações de equivalência em $G^{(0)}$ e grupoides principais em $G^{(0)}$. Devido a arbitrariedade da definição de morfismos dada uma relação de equivalência é sempre possível construir um grupoide principal $G$ que consiste de elementos $\gamma: x \rightarrow y$ para todos os objetos equivalentes $x, y \in G^{(0)}$.

Definição 3.3. Dada uma relação de equivalência $\sim \operatorname{em~} G^{(0)}$, o único grupoide principal $G$ correspondendo a $\sim$ sob a bijeção mencionada é chamado de grupoide principal associado $a \sim$.

Exemplo 3.4. [16] Seja $G^{(0)}=[0,1] \subset \mathbb{R}$ dotado da relação de equivalência trivial, i.e., $x \sim y, \forall x, y \in G^{(0)}$. O gráfico da relação de equivalência é simplesmente $[0,1] \times[0,1]$. Para 
todo $x, y \in G^{(0)}$ existe uma única flecha $\gamma \in G$ tal que $\gamma: y \rightarrow x$. Denote $\gamma=(x, y)$. Observa-se que o mapa $P$ da equação (3.2) é de fato bijetivo, assim se identifica o gráfico de $G \subset G^{(0)} \times G^{(0)}$ com o grupoide $G$. Os mapas fonte e alvo tomam a forma

$$
r(x, y)=x \quad s(x, y)=y
$$

Os elementos $(z, y)$ e $\left(y^{\prime}, x\right)$ podem ser multiplicados caso $y=y^{\prime}$, neste caso

$$
(z, y) \circ\left(y^{\prime}, x\right)=(z, x)
$$

Os elementos identidade de $G$ são identificados com elementos da forma $(y, y)$. A inversa de $(x, y)$ é $(y, x)$.

Exemplo 3.5. Seja $G^{(0)}=[0,1]$ novamente e seja $A \subset G^{(0)}$ conjunto qualquer. Define-se a relação de equivalência $x \sim y$ sse $x=y$ ou $x \in A$ e $y \in A$. Seja $G$ o grupoide principal associado a $\sim$. Note que o gráfico de $G$ é igual a $A \times A \cup D$, onde $D \subset[0,1] \times[0,1]$ é a diagonal.

De um modo geral seja $\mathcal{P}$ partição de $[0,1], \sim$ a relação de equivalência induzida pela partição (dois elementos são equivalentes sse pertencem ao mesmo elemento da partição) e $G$ o grupoide principal associado a relação de equivalência. O gráfico de $G \subset[0,1] \times[0,1]$ é

$$
\bigcup_{A \in \mathcal{P}} A \times A \subset[0,1] \times[0,1]
$$

Exemplo 3.6. [16] Considere a partição do toro $\mathbb{T}^{2}$ em linhas de inclinação irracional $\alpha$. Defina a relação de equivalência $\sim$ induzida pela partição. O grupoide principal associado a $G$ pode ser identificado como

$$
G=\left\{(x, y, \hat{x}, \hat{y}) \in \mathbb{T}^{2} \times \mathbb{T}^{2} \mid \hat{y}-y=\alpha(\hat{x}-x)+\alpha k \quad \bmod 1, k \in \mathbb{Z}\right\}
$$

Neste caso o conjunto das unidades é a diagonal $\mathbb{T}^{2} \subset \mathbb{T}^{2} \times \mathbb{T}^{2}$. O grupoide $G$ também pode ser entendido como $\mathbb{T}^{2} \times \mathbb{R}$ com o conjunto de unidades igual a $\mathbb{T}^{2} \times\{0\}$ da seguinte forma

$$
\begin{aligned}
s: \mathbb{T}^{2} \times \mathbb{R} & \rightarrow \mathbb{T}^{2} \times\{0\} \\
(x, y, z) & \mapsto(x+z, y+\alpha z, 0) \bmod 1 \\
r: \mathbb{T}^{2} \times \mathbb{R} & \rightarrow \mathbb{T}^{2} \times\{0\} \\
(x, y, z) & \mapsto(x-z, y-\alpha z, 0) \bmod 1
\end{aligned}
$$

onde mod 1 age sobre todos os elementos do vetor. É simples verificar que para quaisquer dois pontos $p, q \in \mathbb{T}^{2}$ na mesma partição existe um único ponto $t \in \mathbb{T}^{2} \times \mathbb{R}$ tal que $s(t)=p$ e $r(t)=q$. 


\subsection{Grupoides de Medida}

Definição 3.7. Um grupoide de medida é um grupoide $G$ dotado de uma $\sigma$-álgebra $\mathbb{G}$ de tal sorte que os singletons (pontos) são conjuntos mensuráveis e as seguintes aplicações são mensuráveis

$$
\begin{aligned}
i n v: G & \rightarrow G \\
\gamma & \mapsto \gamma^{-1} \\
\circ: G^{(2)} & \rightarrow G \\
\left(\gamma, \gamma^{\prime}\right) & \mapsto \gamma^{\prime} \gamma \\
r, s: G & \rightarrow G^{(0)}
\end{aligned}
$$

onde a $\sigma$-álgebra de $G \times G$ é $\mathbb{G} \otimes \mathbb{G}$. E as $\sigma$-álgebras de $G^{(0)}$ e $G^{(2)}$ são as induzidas pela inclusão $G^{(0)} \subset G$ e $G^{(2)} \subset G \times G$.

Exemplo 3.8. O grupoide $G$ do exemplo 3.6 com a $\sigma$-álgebra usual de $\mathbb{T}$ e $\mathbb{R}$ é um grupoide de medida.

Definição 3.9. Seja $G$ um grupoide associado a uma relação de equivalência $\sim$ definida em um espaço mensurável $\left(G^{(0)}, \mathbb{G}^{(0)}\right)$. Seja $\mathbb{G} \sigma$-álgebra gerada por conjuntos da forma $s^{-1}(A) \cap$ $r^{-1}(B)$, com $A, B \in \mathbb{G}^{(0)}$. O espaço mensurável $(G, \mathbb{G})$ é chamado de grupoide principal associado, ou simplesmente grupoide associado (em inglês principal associated grupoid) a relação $\sim$.

Sejam $\pi_{1}$ e $\pi_{2}$ projeções de $G^{(0)} \times G^{(0)}$ na primeira e segunda variável respectivamente. Observa-se que $s^{-1}(A) \cap r^{-1}(B)=\pi_{1}^{-1}(A) \cap \pi_{2}^{-1}(B) \cap G$. Por esta observação é natural afirmar que a $\sigma$-álgebra de um grupoide associado é precisamente a mesma $\sigma$-álgebra induzida sobre o grupoide pela inclusão $G \subset G^{(0)} \times G^{(0)}$. Isto realmente é verdadeiro porém há um detalhe. $\mathrm{Na}$ definição de grupoide de medida o contra-domínio das funções $r, s$ deve ser entendido como a diagonal $G^{(0)} \subset G$ e não com os "eixos". Assim para fazer a identificação com os operadores projeção é necessário primeiro mostrar que a $\sigma$-álgebra induzida em $G^{(0)}$ pela inclusão da diagonal em $G$, i.e., a $\sigma$-álgebra induzida pela inclusão $G^{(0)} \subset G$ pode ser identificada com $\mathbb{G}^{(0)}$ a $\sigma$-álgebra nos "eixos". Para isso utiliza-se o seguinte lema

Lema 3.10. Seja $X$ um conjunto, e seja $\mathfrak{B} \subset 2^{X}$ uma coleção de subconjuntos de $X$. Seja $G \subset X$. A seguinte igualdade é verdadeira

$$
G \cap \sigma(\mathfrak{B})=\sigma(G \cap \mathfrak{B})
$$

onde $\sigma(\mathfrak{B})$ denota a $\sigma$-álgebra gerada por $\mathfrak{B} . G \cap \sigma(\mathfrak{B})$ é a $\sigma$-álgebra induzida em $G$ por $\sigma(\mathfrak{B})$ também chamada de $\sigma$-álgebra traço em $G$ e $G \cap \mathfrak{B}=\{A \subset X \mid A=G \cap B, B \in \mathfrak{B}\}$.

Continuando seja $D$ a diagonal de $G^{(0)} \times G^{(0)}$, note que $D \subset G$. Assim 


$$
\begin{gathered}
D \cap\left(G \cap \sigma\left(\{A \times B\}_{A, B \in \mathbb{G}^{(0)}}\right)\right)=D \cap \sigma\left(G \cap\{A \times B\}_{A, B \in \mathbb{G}^{(0)}}\right)= \\
\sigma\left(D \cap G \cap\{A \times B\}_{A, B \in \mathbb{G}^{(0)}}\right)=\sigma\left(D \cap\{A \times B\}_{A, B \in \mathbb{G}^{(0)}}\right)= \\
\sigma\left(D \cap\{A \cap B \times A \cap B\}_{A, B \in \mathbb{G}^{(0)}}\right)=\sigma\left(D \cap\{A \times A\}_{A \in \mathbb{G}^{(0)}}\right)
\end{gathered}
$$

Nota-se que o último termo é facilmente identificado com $\sigma\left(\{A\}_{A \in \mathbb{G}^{(0)}}\right)=\mathbb{G}^{(0)}$ como queria demonstrar. Assim a $\sigma$-álgebra induzida sobre a diagonal de $G$ pode ser identificada com a $\sigma$-álgebra em $\mathbb{G}^{(0)}$. E portanto pode-se identificar $r, s$ com projeções restritas a $G$.

Deste resultado segue a próxima proposição que mostra, ao contrário do que afirma [16], que todo grupoide associado a uma relação de equivalência é um grupoide de medida.

Proposição 3.11. Seja $\left(G^{(0)}, \mathbb{G}^{(0)}\right)$ um espaço mensurável, e $\sim$ uma relação de equivalência sobre $G^{(0)}$.O grupoide associado $(G, \mathbb{G})$ é um grupoide de medida.

Demonstração. Seja $\pi: G^{(0)} \times G^{(0)} \rightarrow G^{(0)}$ a projeção na segunda variável. Tem-se que $s: G \rightarrow G^{(0)}$ é claramente mensurável pois $s^{-1}(A)=G \cap \pi^{-1}(A)$ que é um subconjunto mensurável da $\sigma$-álgebra induzida por $\mathbb{G}^{(0)} \otimes \mathbb{G}^{(0)}$ sobre $G$ sempre que $A \in \mathbb{G}^{(0)}$. Argumento similar se aplica a $r: G \rightarrow G^{(0)}$.

Para a inversa note que pelo lema 3.10 e pela definição de $\mathbb{G}^{(0)} \otimes \mathbb{G}^{(0)}$, conjuntos da forma $B=s^{-1}\left(A_{1}\right) \cap r^{-1}\left(A_{2}\right), A_{1}, A_{2} \in \mathbb{G}^{(0)}$ geram $\mathbb{G}$ e observe que

$$
\text { inv }: B \rightarrow B^{\prime}=s^{-1}\left(A_{2}\right) \cap r^{-1}\left(A_{1}\right)
$$

e $B^{\prime}$ pertence a família geradora, logo inv é uma função mensurável.

Para a multiplicação observa-se

$$
\circ^{-1}(B)=G^{(2)} \cap\left(s^{-1}\left(A_{1}\right) \times r^{-1}\left(A_{2}\right)\right) \subset G^{(2)} \cap G \times G
$$

e claramente $G^{(2)} \cap\left(s^{-1}\left(A_{1}\right) \times r^{-1}\left(A_{2}\right)\right)$ é um subconjunto mensurável da $\sigma$-álgebra induzida em $G^{(2)}$ por $G \times G$. Logo o é função mensurável.

Definição 3.12. Quando um grupoide principal associado $G$ for de tal sorte que $G$ pode ser identificado com um subconjunto mensurável de $G^{(0)} \times G^{(0)}$ então $G$ é dito grupoide principal compatível, ou simplesmente grupoide compatível .

Corolario 3.13. O grupoide principal compativel $G$ a uma relação de equivalência em um espaço standart $G^{(0)}$ é um grupoide de medida sse $G$ é um espaço standart [16].

\subsection{Kernel}

Dado um espaço mensurável $(Y, \mathbb{Y})$ define-se $\overline{\mathcal{F}}^{+}(Y)=\left\{f: Y \rightarrow \overline{\mathbb{R}}^{+} \mid f\right.$ é mensurável $\}$ e $\mathcal{F}^{+}(Y)=\left\{f: Y \rightarrow \mathbb{R}^{+} \mid f\right.$ é mensurável $\}$. 
Definição 3.14. Dados dois espaços mensuráveis $(Y, \mathbb{Y})$ e $\left(Y^{\prime}, \mathbb{Y}^{\prime}\right)$ um kernel $\lambda$ de $Y$ a $Y^{\prime}$ é uma função $\lambda: \overline{\mathcal{F}}^{+}(Y) \rightarrow \overline{\mathcal{F}}^{+}\left(Y^{\prime}\right)$, denotada por $\lambda: Y \rightarrow Y^{\prime}$, que satisfaz as propriedades

(i) Linearidade, i.e., $\lambda(\alpha f+\beta g)=\alpha \lambda(f)+\beta \lambda(g), \forall \alpha, \beta \in \overline{\mathbb{R}}^{+}, f, g \in \overline{\mathcal{F}}^{+}(Y)$;

(ii) Normalidade, i.e., se $\left(f_{n}\right)_{n \in \mathbb{N}}$ é sequência de elementos de $\overline{\mathcal{F}}^{+}$tal que $f_{n} \nearrow f$, isto implica que $\lambda\left(f_{n}\right) \nearrow \lambda(f)$.

Denote por $\overline{\mathbb{M}}^{+}(Y)$ o conjunto de medidas positivas de $Y$. Um kernel $\lambda$ é completamente determinado pela função

$$
\begin{aligned}
Y^{\prime} & \rightarrow \overline{\mathbb{M}}^{+}(Y) \\
y^{\prime} & \mapsto \lambda^{y^{\prime}}
\end{aligned}
$$

com $\lambda^{y^{\prime}}(S)=\lambda\left(\chi_{S}\right)\left(y^{\prime}\right), S \in \mathbb{Y}$. Para ver isso basta notar que uma função qualquer $f \in$ $\overline{\mathcal{F}}^{+}(Y)$ pode ser escrita como o limite pontual de uma sequência crescente de funções simples e aplica-se então as propriedades de kernel. Formalmente seja $\varphi_{n}$ sequência de funções simples tais que $\varphi_{n} \nearrow f$, tem-se que

$$
\begin{gathered}
\lambda(f)\left(y^{\prime}\right)=\lambda\left(\lim _{n} \varphi_{n}\right)\left(y^{\prime}\right)=\lambda\left(\lim _{n} \sum_{i} a_{i n} \chi_{A_{i n}}\right)\left(y^{\prime}\right)= \\
\lim _{n} \lambda\left(\sum_{i} a_{i n} \chi_{A_{i n}}\right)\left(y^{\prime}\right)=\lim _{n} \sum_{i} a_{i n} \lambda^{y^{\prime}}\left(A_{i n}\right)= \\
\lim _{n} \int \varphi d \lambda^{y^{\prime}}(y)=\int f(y) d \lambda^{y^{\prime}}(y)=\lambda^{y^{\prime}}(f)
\end{gathered}
$$

com $\lambda^{y^{\prime}}(f)$ sendo a integral da função $f$ segundo a medida $\lambda^{y^{\prime}}$, i.e., $\int f(y) d \lambda^{y^{\prime}}(y) \equiv \lambda^{y^{\prime}}(f)$.

Dado um grupoide $G$, um conjunto $A \subset G$ e uma flecha $\gamma \in G$. A multiplicação a esquerda de $A$ por $\gamma, \gamma A$, é definida pela igualdade

$$
\gamma A=\left\{\gamma^{\prime} \in G \mid \gamma^{\prime}=\gamma \gamma^{\prime \prime} \text { para algum } \gamma^{\prime \prime} \in A \text { que satisfaça } r\left(\gamma^{\prime \prime}\right)=s(\gamma)\right\}
$$

De modo análogo se define a multiplicação a direita, $A \gamma$.

Exemplo 3.15. Seja $(X, \mathbb{X}, \mu)$ um espaço métrico compacto com medida de probabilidade boreliana e $\xi$ uma partição mensurável. Seja $\left\{\mu_{C}\right\}_{C \in X / \xi}$ desintegração de $\mu$ com respeito a partição $\xi$. Define-se o kernel $\lambda: X \rightarrow X / \xi$ por

$$
(\lambda(f))(C)=\int_{C \subset X} f d \mu_{C}
$$

Segue da definição de desintegração que $\lambda(f)$ é função mensurável em $X / \xi$.

Definição 3.16. Suponha que $\pi: Y \rightarrow Y^{\prime}$ é uma sobrejeção mensurável. Diz-se que o kernel $\lambda: \overline{\mathcal{F}}^{+}(Y) \rightarrow \overline{\mathcal{F}}^{+}\left(Y^{\prime}\right)$ é carregado, ou fibrado, por $\pi$ se para cada $y^{\prime} \in Y^{\prime}$, a medida $\lambda^{y^{\prime}}$ é tal que $\lambda^{y^{\prime}}(A)=\lambda^{y^{\prime}}\left(A \cap \pi^{-1}\left(y^{\prime}\right)\right)$, para todo $A \subset Y$ mensurável. 
Proposição 3.17. Seja $\lambda: Y \rightarrow Y^{\prime}$ kernel, $\lambda$ é fibrado por $\pi$ sse para cada $f \in \overline{\mathcal{F}}^{+}(Y)$ e $g \in \overline{\mathcal{F}}^{+}\left(Y^{\prime}\right)$ tem-se que

$$
\lambda(f \cdot(g \circ \pi))=g \cdot \lambda(f)
$$

onde o . indica a multiplicação usual em $\overline{\mathbb{R}}$.

Demonstração. A prova é consequência direta da forma integral de $\lambda$.

Um kernel $\lambda: Y \rightarrow Y^{\prime}$ é chamado de $\sigma$-finito (respectivamente próprio) sempre que é possível exibir uma sequência $\left(B_{n}\right)_{n \in \mathbb{N}}$ de conjuntos mensuráveis de $Y$ tais que $\bigcup_{n \in \mathbb{N}} B_{n}=$ $Y$ e $\lambda\left(\chi_{B_{n}}\right)$ é função finita (respectivamente limitada) para todo $n \in \mathbb{N}$. É simples ver que a existência de uma função não nula $f \in \overline{\mathcal{F}}^{+} \operatorname{com} \lambda(f)$ finita (respectivamente limitada) implica que $\lambda$ é $\sigma$-finito (respectivamente significa que é próprio). Para isso defina $B_{0}=\{y \in Y \mid f(y) \geq 1\}$ e $B_{n}=\left\{y \in Y \mid 2^{-n+1}>f(y) \geq 2^{-n}\right\}$ para todo $n>0$. Assim $f \geq \sum_{n} 2^{-n} \chi_{B_{n}}(x)$, donde seguem os resultados. O símbolo $\mathcal{C}^{+}$é designado para denotar o conjunto de todos os kernels próprios de um dado grupoide.

Definição 3.18. Seja $G$ um grupoide de medida. Um G-kernel é um kernel $\lambda: G \rightarrow G^{(0)}$ fibrado pelo mapa alvo, $r: G \rightarrow G^{(0)}$.

Define-se a convolução de um $G$-kernel $\lambda$ e uma função $f \in \overline{\mathcal{F}}^{+}$, como sendo a função $\lambda * f$ dada por:

$$
(\lambda * f)(\gamma)=\int f\left(\gamma^{\prime-1} \gamma\right) d \lambda^{r(\gamma)}\left(\gamma^{\prime}\right), \quad \gamma \in G
$$

Nota-se que a multiplicação $\gamma^{\prime-1} \gamma$ só faz sentido para flechas $\gamma^{\prime}$ tal que $r\left(\gamma^{\prime}\right)=r(\gamma)$, ou seja só faz sentido para flechas contidas em $r^{-1}(\gamma)$. Observação análoga é válida para operações similares.

Define-se o produto $f \lambda$ como sendo o kernel tal que $(f \lambda)(g)=\lambda(f g), \forall g \in \overline{\mathcal{F}}^{+}(G)$. E define-se a convolução entre duas funções $f, g$ segundo um kernel $\lambda$ por

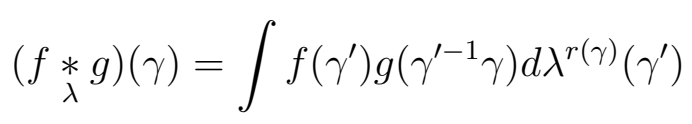

é fácil ver que $\underset{\lambda}{f * g}=f \lambda * g$.

A próxima proposição devida a Kastler, traz algumas relações úteis entre os objetos matemáticos definidos neste capítulo até o presente momento. A sua demonstração exemplifica os cálculos envolvendo funções e kernels.

Proposição 3.19. [7] Seja $\lambda$ um G-kernel e $f \in \overline{\mathcal{F}}^{+}(G)$, define-se

$$
\begin{gathered}
\left(L_{\lambda} f\right)(\gamma)=\int f\left(\gamma^{\prime-1} \gamma\right) d \lambda^{r(\gamma)}\left(\gamma^{\prime}\right)=(\lambda * f)(\gamma) \\
\left(R_{\lambda} f\right)(\gamma)=\int f\left(\gamma \gamma^{\prime}\right) d \lambda^{s(\gamma)}\left(\gamma^{\prime}\right)
\end{gathered}
$$


(i) Se $\lambda$ é $\sigma$-finito $L_{\lambda}, R_{\lambda}$ são kernels de $G$ em $G$ relacionados por

$$
L_{\lambda} f=\left(R_{\lambda} \tilde{f}\right)^{\sim}, f \in \overline{\mathcal{F}}^{+}(G)
$$

$\operatorname{com} \tilde{f}(\gamma)=f\left(\gamma^{-1}\right), \gamma \in G$

(ii) $\lambda(\tilde{f})=\left.(\lambda * f)\right|_{G^{(0)}}, \forall f \in \overline{\mathcal{F}}^{+}(G)$

(iii) $L_{\lambda} L_{\mu}=L_{\lambda R_{\mu}}$

(iv) Para $f \in \overline{\mathcal{F}}^{+}(G)$ e $\varphi \in \overline{\mathcal{F}}^{+}\left(G^{(0)}\right)$ tem-se

$$
\begin{aligned}
& R_{\lambda}((\varphi \circ r) f)=(\varphi \circ r) R_{\lambda} f \\
& L_{\lambda}((\varphi \circ r) f)=(\varphi \circ s) L_{\lambda} f,
\end{aligned}
$$

(v) Se $\lambda$ e $\mu$ são kernels $\sigma$-finitos então $\lambda(\mu * f)=\mu(\lambda * \tilde{f}), f \in \overline{\mathcal{F}}^{+}(G)$

Observa-se que o item (iii) sugere a seguinte notação para a convolução de dois kernels

$$
\lambda * \mu:=\lambda R_{\mu}
$$

e de (iii) segue a seguinte propriedade

$$
\lambda *(\mu * f)=(\lambda * \mu) * f, f \in \overline{\mathcal{F}}^{+}(G) .
$$

Com o objetivo de simplificar a notação daqui em diante a integral de uma função $f$ em relação a uma medida $\mu$ poderá ser representada como $\mu(f)$, i.e., $\mu(f)=\int f d \mu$. Em particular se $B$ é um subconjunto mensurável então $\mu\left(\chi_{B}\right)=\mu(B)$.

Demonstração. ( $i$ ) É fácil ver que $L_{\lambda}, R_{\lambda}$ são lineares e normais, a prova da mensurabilidade depende de $\lambda$ ser $\sigma$-finito, esta prova é relativamente técnica e sua demonstração será omitida aqui, o leitor interessador pode consultar [7] para a demonstração. Segue a demonstração da igualdade

$$
\begin{gathered}
\left(R_{\lambda} \tilde{f}\right)^{\sim}(\gamma)=\left(R_{\lambda} \tilde{f}\right)\left(\gamma^{-1}\right)=\int \tilde{f}\left(\gamma^{-1} \gamma^{\prime}\right) d \lambda^{s\left(\gamma^{-1}\right)}\left(\gamma^{\prime}\right)= \\
\int f\left(\gamma^{\prime-1} \gamma\right) d \lambda^{r(\gamma)}\left(\gamma^{\prime}\right)=\left(L_{\lambda} f\right)(\gamma), \forall \gamma \in G .
\end{gathered}
$$

(ii) Lembra-se que $\gamma \in G^{(0)} \subset G \Leftrightarrow \gamma=\mathbb{1}_{x}$ para algum $x \in G^{(0)}$. Assim em particular se $\gamma^{\prime}: s\left(\gamma^{\prime}\right) \rightarrow r(\gamma)$, logo $\gamma^{\prime-1} \gamma=\gamma^{\prime-1}$. Assim para $S \subset G$ mensurável tem-se que

$$
(\lambda * f)\left(\mathbb{1}_{x}\right)=\left(L_{\lambda} f\right)\left(\mathbb{1}_{x}\right)=\int f\left(\gamma^{\prime-1} \mathbb{1}_{x}\right) d \lambda^{x}\left(\gamma^{\prime}\right)=\int \tilde{f}\left(\gamma^{\prime}\right) d \lambda^{x}\left(\gamma^{\prime}\right)=\lambda(\tilde{f})(x)
$$

donde segue a igualdade. 
(iii) Tem-se que para qualquer função característica de um conjunto mensurável $S$

$$
\begin{gathered}
\left(\lambda R_{\mu}\right)^{r(\gamma)}\left(\chi_{S}\right)=\left(\lambda \circ R_{\mu}\right)^{r(\gamma)}\left(\chi_{S}\right)=\lambda^{r(\gamma)}\left(R_{\mu} \chi_{S}\right)= \\
\int\left(R_{\mu} \circ \chi_{S}\right)\left(\gamma^{\prime}\right) d \lambda^{r(\gamma)}\left(\gamma^{\prime}\right)=\iint \chi_{S}\left(\gamma^{\prime} \gamma^{\prime \prime}\right) d \mu^{s\left(\gamma^{\prime}\right)}\left(\gamma^{\prime \prime}\right) d \lambda^{r(\gamma)}\left(\gamma^{\prime}\right)= \\
\iint \chi_{\gamma^{\prime-1} S}\left(\gamma^{\prime \prime}\right) d \mu^{s\left(\gamma^{\prime}\right)}\left(\gamma^{\prime \prime}\right) d \lambda^{r(\gamma)}\left(\gamma^{\prime}\right)=\int \mu^{s\left(\gamma^{\prime}\right)}\left(\gamma^{\prime-1} S\right) d \lambda^{r(\gamma)}\left(\gamma^{\prime}\right)
\end{gathered}
$$

E portanto

$$
\begin{gathered}
L_{\lambda R_{\mu}}\left(\chi_{S}\right)(\gamma)=\int \chi_{S}\left(\gamma^{\prime-1} \gamma\right) d\left(\lambda R_{\mu}\right)^{r(\gamma)}\left(\gamma^{\prime}\right)= \\
\int \chi_{\gamma S^{-1}}\left(\gamma^{\prime}\right) d\left(\lambda R_{\mu}\right)^{r(\gamma)}\left(\gamma^{\prime}\right)=\left(\lambda R_{\mu}\right)^{r(\gamma)}\left(\gamma S^{-1}\right)= \\
\int \mu^{s\left(\gamma^{\prime}\right)}\left(\gamma^{\prime-1} \gamma S^{-1}\right) d \lambda^{r(\gamma)}\left(\gamma^{\prime}\right) .
\end{gathered}
$$

Por outro lado

$$
\begin{aligned}
& \left(L_{\lambda}\left(L_{\mu}\left(\chi_{S}\right)\right)\right)(\gamma)=\int L_{\mu}\left(\chi_{S}\right)\left(\gamma^{\prime-1} \gamma\right) d \lambda^{r(\gamma)}\left(\gamma^{\prime}\right)= \\
& \iint \chi_{S}\left(\gamma^{\prime \prime-1} \gamma^{\prime-1} \gamma\right) d \mu^{r\left(\gamma^{\prime-1} \gamma\right)}\left(\gamma^{\prime \prime}\right) d \lambda^{r(\gamma)}\left(\gamma^{\prime}\right)= \\
& \iint \chi_{S}\left(\gamma^{\prime \prime-1} \gamma^{\prime-1} \gamma\right) d \mu^{s\left(\gamma^{\prime}\right)}\left(\gamma^{\prime \prime}\right) d \lambda^{r(\gamma)}\left(\gamma^{\prime}\right)= \\
& \iint \chi_{\gamma^{\prime-1} \gamma S^{-1}}\left(\gamma^{\prime \prime}\right) d \mu^{s\left(\gamma^{\prime}\right)\left(\gamma^{\prime \prime}\right)}\left(\gamma^{\prime}\right) d \lambda^{r(\gamma)}\left(\gamma^{\prime}\right)= \\
& \int \mu^{s\left(\gamma^{\prime}\right)}\left(\gamma^{\prime-1} \gamma S^{-1}\right) d \lambda^{r(\gamma)}\left(\gamma^{\prime \prime}\right)
\end{aligned}
$$

Comparando (3.6) e (3.7) segue a conclusão para funções características. Da linearidade e normalidade segue que o resultado é válido para qualquer função mensurável.

$(i v)$

$$
\begin{gathered}
R_{\lambda}((\varphi \circ r) f)(\gamma)=\int((\varphi \circ r) f)\left(\gamma \gamma^{\prime}\right) d \lambda^{s(\gamma)}\left(\gamma^{\prime}\right)= \\
\int(\varphi \circ r)(\gamma) f\left(\gamma \gamma^{\prime}\right) d \lambda^{r(\gamma)}\left(\gamma^{\prime}\right)=(\varphi \circ r) R_{\lambda} f
\end{gathered}
$$

Procede-se analogamente para $L_{\lambda}$.

$(v)$

$$
\begin{gathered}
\lambda^{y}(\mu * f)=\iint f\left(\gamma^{\prime-1} \gamma\right) d \mu^{r(\gamma)}\left(\gamma^{\prime}\right) d \lambda^{y}(\gamma)= \\
\iint f\left(\gamma^{\prime-1} \gamma\right) d \mu^{y}\left(\gamma^{\prime}\right) d \lambda^{y}(\gamma)=\iint f\left(\gamma^{\prime-1} \gamma\right) d \lambda^{y}(\gamma) d \mu^{y}\left(\gamma^{\prime}\right)= \\
\iint \tilde{f}\left(\gamma^{-1} \gamma^{\prime}\right) d \lambda^{y}\left(\gamma^{\prime}\right)=\mu^{y}(\lambda * \tilde{f})
\end{gathered}
$$


Donde se obtem a conclusão, uma vez que é licito trocar a ordem de integração pois $\mu$ e $\lambda$ são $\sigma$-finitos.

Definição 3.20. Dado um $G$-kernel $\lambda: G \rightarrow G^{(0)}$ define-se o suporte de $\lambda$, $S_{\lambda}$, pela igualdade $S_{\lambda}=\left\{x \in G^{(0)} \mid \lambda^{x} \neq 0\right\}$. O $G$-kernel $\lambda$ é dito fiel caso $S_{\lambda}=G^{(0)}$.

A próxima proposição também devida a Kastler mostra que para $G$-kernels ser $\sigma$-finito é equivalente a ser próprio. Além disso se $\lambda$ for $G$-kernel com esta propriedade garante-se a existência de uma função $f$, tal que $\lambda$ avaliado em $f$ é a função característica do suporte de $\lambda$.

Proposição 3.21. [7] Seja $G$ um grupoide de medida e seja $\lambda$ um G-kernel. As seguintes afirmações são equivalentes:

(i) $\lambda$ é $\sigma$-finito,

(ii) Existe função estritamente positiva (i.e. $f(\gamma) \neq 0, \forall \gamma \in G) f \in \overline{\mathcal{F}}^{+}(G)$ com $\lambda(f)$ finita.

(iii) Exite função estritamente positiva $f \in \overline{\mathcal{F}}^{+}(G)$ com $\lambda(f)$ limitada.

(iv) $\lambda$ é próprio.

Além disso se alguma destas condições é satisfeitas as funções nos itens (ii) e (iii) podem ser escolhidas limitadas e $\exists g \in \overline{\mathcal{F}}^{+}(G)$ tal que

$$
\lambda(g)=\chi_{S_{\lambda}}, S_{\lambda}=\left\{x \in G^{(0)} \mid \lambda^{x} \neq 0\right\}
$$

Demonstração. $(i v) \Leftrightarrow(i i i)$

$(\Rightarrow)$ Da hipótese de ser próprio segue que existe sequência $\left(B_{n}\right)_{n \in \mathbb{N}}$ de subconjuntos mensuráveis de $G$ tais que $\cup_{n} B_{n}=G$ e $\lambda\left(B_{n}\right)<M(n)$ para cada $n$. Assim defina $f: G \rightarrow \mathbb{R}$ por

$$
f(\gamma)=\sum_{n=0}^{\infty} \frac{\chi_{B_{n}}(\gamma)}{M(n)} \frac{1}{2^{n}}
$$

segue diretamente da definição que $f$ é mensurável e estritamente positiva. E tem-se que

$$
\lambda(f)(x)=\sum_{n=0}^{\infty} \frac{\lambda^{x}\left(\chi_{b_{n}}\right)}{M(n)} \frac{1}{2^{n}} \leq \sum_{n=0}^{\infty} \frac{1}{2^{n}}=1, \forall x \in G^{(0)}
$$

$(\Leftarrow)$ Feita anteriormente.

(iii) $\Rightarrow$ (ii) Trivial.

$(i i) \Rightarrow(i)$ Análoga a $(i i i) \Rightarrow(i v)$. 
$(i) \Rightarrow\left(\right.$ iii) Para $n, m \in \mathbb{N}, A_{m, n}=\left\{y \in G^{(0)} \mid \lambda^{y}\left(B_{n}\right)<m\right\}$. Para $n$ fixo $A_{m, n}$ é sequência crescente de conjuntos e $\cup_{m} A_{m, n}=G^{(0)}$, assim $r^{-1}\left(A_{m, n}\right)$ é crescente em direção de de cobrir o conjunto $G$. Assim $B_{m, n}^{\prime}=B_{n} \cap r^{-1}\left(A_{m, n}\right)$ são conjuntos mensuráveis que cobrem $G$ tais que $\lambda\left(B_{m, n}^{\prime}\right)<m$. A função $f=\sum_{m, n} m^{-1} 2^{-(n+m)} \chi_{B_{m, n}^{\prime}} \operatorname{satisfaz~}($ iii $)$.

Para provar a existência da $g$, escolha $f$ como em $(i i)$ e define-se $g=(\varphi \circ r) f$, com $\varphi(y)=\lambda^{y}(f)^{-1}$ para $y \in S_{\lambda}$ e $\varphi=1$ para $y \notin S_{\lambda}$. Observa-se que $S_{\lambda}$ é mensurável e que se $y \in S_{\lambda}$, $\log \lambda^{y}(f)>0$ pois $\lambda^{y} \neq 0$ e $f(\gamma) \neq 0, \forall \gamma \in G$. Assim tem-se que

$$
\lambda(g)=\lambda((\varphi \circ r) f)=(\varphi \circ r) \lambda(f)=\chi_{S_{\lambda}}
$$

\subsection{Funções Transversas}

Funções transversas são $G$-kernels que são invariantes pela ação do grupoide. São análogas a medida de Haar sobre um grupo. De fato coincidem com a medida de Haar quando o grupoide é um grupo. Kastler utiliza o seguinte resultado para caracterizar funções transversas.

Proposição 3.22. [7] Seja G grupoide mensurável e seja $\nu$ um G-kernel. As seguintes afirmações são equivalentes:

(i) $\nu^{r(\gamma)}=\gamma \nu^{s(\gamma)}, \gamma \in G$, i.e., $\nu^{r(\gamma)}(A)=\nu^{s(\gamma)}\left(\gamma^{-1} A\right), \forall A$ mensurável e $\gamma \in G$;

(ii) $\nu * f=\nu(\tilde{f}) \circ s, f \in \overline{\mathcal{F}}^{+}(G)$,

(iii) $(f \underset{\nu}{*} g)^{\sim}=\tilde{g} * \underset{\nu}{\tilde{f}}, f, g \in \overline{\mathcal{F}}^{+}(G)$;

(iv) $\lambda * f \nu=(\lambda * f) \nu, f \in \overline{\mathcal{F}}^{+}(G)$, com $\lambda$ um G-kernel $\sigma$-finito qualquer.

Se qualquer uma das condições for satisfeita $\underset{\nu}{*}$ é associativo.

Demonstração. Primeiramente prova-se que

$$
\begin{gathered}
\nu^{r(\gamma)}(S)=\nu^{s(\gamma)}\left(\gamma^{-1} S\right), \forall S \text { mensurável e } \gamma \in G . \\
\text { ocorre se, e somente se, } \\
\int f\left(\gamma^{\prime}\right) d \nu^{r(\gamma)}(\gamma)=\int f\left(\gamma \gamma^{\prime}\right) d \nu^{s(\gamma)}\left(\gamma^{\prime}\right), \forall f \in \overline{\mathcal{F}}^{+}(G), \gamma \in G
\end{gathered}
$$

Para isso assume-se a priori a segunda proposição e prova-se a primeira, seja $S$ subconjunto mensurável de $G$ tem-se que

$$
\begin{aligned}
\nu^{r(\gamma)}(S)= & \int \chi_{S}\left(\gamma^{\prime}\right) d \nu^{r(\gamma)}\left(\gamma^{\prime}\right)=\int \chi_{S}\left(\gamma \gamma^{\prime}\right) d \nu^{s(\gamma)}\left(\gamma^{\prime}\right)= \\
& \int \chi_{\gamma^{-1} S}\left(\gamma^{\prime}\right) d \nu^{s(\gamma)}\left(\gamma^{\prime}\right)=\nu^{s(\gamma)}\left(\gamma^{-1} S\right) .
\end{aligned}
$$


Por outro lado

$$
\int \chi_{S}\left(\gamma^{\prime}\right) d \nu^{r(\gamma)}\left(\gamma^{\prime}\right)=\nu^{r(\gamma)}(S)=\nu^{s(\gamma)}\left(\gamma^{-1} S\right)=\int \chi_{S}\left(\gamma \gamma^{\prime}\right) d \nu^{s(\gamma)}\left(\gamma^{\prime}\right)
$$

Assim (3.8) está provada. Procede-se a demonstração.

$(i) \Leftrightarrow(i i)$ Nota-se que

$$
(\nu * f)(\gamma)=\int f\left(\gamma^{\prime-1} \gamma\right) d \nu^{r(\gamma)}\left(\gamma^{\prime}\right)=\int \tilde{f}\left(\gamma^{-1} \gamma^{\prime}\right) d \nu^{r(\gamma)}\left(\gamma^{\prime}\right)
$$

e

$$
(\nu(\tilde{f}) \circ s)(\gamma)=\int \tilde{f}\left(\gamma^{\prime}\right) d \nu^{s(\gamma)}\left(\gamma^{\prime}\right)
$$

A igualdade segue da equivalência (3.8) pois

$$
\int \tilde{f}\left(\gamma^{-1} \gamma^{\prime}\right) d \nu^{r(\gamma)}\left(\gamma^{\prime}\right)=\int \tilde{f}\left(\gamma \gamma^{-1} \gamma^{\prime}\right) d \nu^{s(\gamma)}\left(\gamma^{\prime}\right)=\int \tilde{f}\left(\gamma^{\prime}\right) d \nu^{s(\gamma)}\left(\gamma^{\prime}\right)
$$

$(i) \Leftrightarrow($ iii) Da mesma forma nota-se que

$$
\begin{gathered}
(\underset{\nu}{*} g)^{\sim}(\gamma)=\int f\left(\gamma^{\prime}\right) g\left(\gamma^{\prime-1} \gamma^{-1}\right) d \nu^{s(\gamma)}\left(\gamma^{\prime}\right) \\
(\underset{\nu}{\tilde{g} * \tilde{f}})(\gamma)=\int \tilde{g}\left(\gamma^{\prime}\right) \tilde{f}\left(\gamma^{\prime-1} \gamma\right) d \nu^{r(\gamma)}\left(\gamma^{\prime}\right)=\int g\left(\gamma^{\prime-1}\right) f\left(\gamma^{-1} \gamma^{\prime}\right) d \nu^{r(\gamma)}\left(\gamma^{\prime}\right)
\end{gathered}
$$

Mas pela equivalência (3.8) segue que

$$
\begin{aligned}
& \int \tilde{g}\left(\gamma^{\prime}\right) f\left(\gamma^{-1} \gamma^{\prime}\right) d \nu^{r(\gamma)}\left(\gamma^{\prime}\right)=\int \tilde{g}\left(\gamma \gamma^{\prime}\right) f\left(\gamma \gamma^{-1} \gamma^{\prime}\right) d \nu^{s(\gamma)}\left(\gamma^{\prime}\right)=\int f\left(\gamma^{\prime}\right) g\left(\gamma^{\prime-1} \gamma^{-1}\right) d \nu^{s(\gamma)}\left(\gamma^{\prime}\right) \\
& (\text { iii }) \Leftrightarrow(i v)
\end{aligned}
$$

Primeiramente prova-se que se $p, q \in \overline{\mathcal{F}}^{+}(G)$ forem tais que $\lambda(p)=\lambda(q)$, para todo Gkernel $\lambda$ então $p=q$. Para isso suponha $p \neq q$, i.e, existe $\gamma_{0} \in G$ tal que $p\left(\gamma_{0}\right) \neq q\left(\gamma_{0}\right)$, defina $\lambda^{r\left(\gamma_{0}\right)}=\delta_{\gamma_{0}}$ e $\lambda^{y}=0$ para todo $y \neq r\left(\gamma_{0}\right)$. Logo

$$
\lambda^{r\left(\gamma_{0}\right)}(p)=\int p(\gamma) d \lambda^{r\left(\gamma_{0}\right)}(\gamma)=p\left(\gamma_{0}\right) \neq q\left(\gamma_{0}\right)=\lambda^{r\left(\gamma_{0}\right)}(q)
$$

a mensurabilidade da imagem de $\lambda$ é devida ao fato de que os singletons são mensuráveis. Em vista deste resultado (iii) é equivalente a provar que

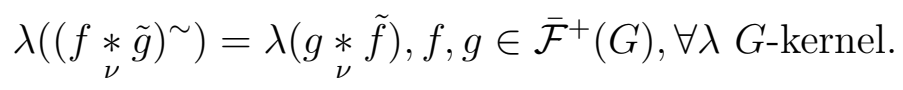

Utilizando os comentários acima procede-se a demonstração.

$$
\begin{gathered}
\lambda\left[(f \underset{\nu}{f} \tilde{g})^{\sim}\right]=\left.\lambda *(\underset{\nu}{f} \tilde{g})\right|_{G^{(0)}}=\left.\lambda *(f \nu * \tilde{g})\right|_{G^{(0)}}= \\
\left.\lambda * f \nu * \tilde{g}\right|_{G^{(0)}}=\left.(\lambda * f \nu) * \tilde{g}\right|_{G^{(0)}}=(\lambda * f \nu)(g)
\end{gathered}
$$


Onde foram utilizados os itens (ii) e (iii) da proposição 3.19. Por outro lado fazendo uso do item $(v)$ de 3.19 tem-se

$$
\lambda(g \nu * \tilde{f})=g \nu(\lambda * f)=[(\lambda * f) \nu](g) .
$$

Dos dois últimos desenvolvimentos tem-se que

$$
\begin{gathered}
\lambda\left((\underset{\nu}{*} \underset{g}{\tilde{g}})^{\sim}\right)=\lambda(\underset{\nu}{*} \underset{f}{f}) \\
\text { se, e somente se } \\
(\lambda * f \nu)(g)=((\lambda * f) \nu)(g)
\end{gathered}
$$

donde segue a equivalência.

$(i v) \Rightarrow$ Associatividade

A associatividade é uma consequência simples de $(i v)$, segue a demonstração.

$$
(f \underset{\nu}{*} g) \underset{\nu}{*} h=(f \nu * g) \nu * h=(f \nu * g \nu) * h=f \nu *(g \nu * h)=\underset{\nu}{f} \underset{\nu}{(g} \underset{\nu}{ } h)
$$

Se um $G$-kernel $\nu$ satisfazer qualquer uma das condições equivalentes da proposição 3.22 então $\nu$ é denominada função transversa. Dado um grupoide $\mathrm{G}$ denota-se por $\mathcal{E}^{+}$o espaço das funções transversas próprias em G. A próxima proposição devida a Connes, mostra que se $\nu_{0} \in \mathcal{E}^{+}$é função transversa fiel então toda a função transversa $\nu \in \mathcal{E}^{+}$pode ser escrita como $\nu=\nu_{0} * \lambda$ com $\lambda$ um $G$-kernel.

Proposição 3.23. [3]

a) Seja $\nu$ uma função transversa e $\lambda$ um G-kernel, então $\nu * \lambda$ é uma função transversa.

b) Seja $\nu_{0} \in \mathcal{E}^{+}$fiel no sentido de que $\nu_{0}^{y} \neq 0$ para todo $y \in G^{(0)}$. Então cada $\nu \in \mathcal{E}^{+}$é da forma $\nu_{0} * \lambda$ com $\lambda$ um G-kernel. Especificamente

$$
\nu=\nu_{0} * \lambda
$$

Demonstração. a) Deve-se provar que $(\nu * \lambda)^{r(\gamma)}=\gamma\left(\nu \circ R_{\lambda}\right)^{s(\gamma)}$. A prova segue da seguinte sequência de cálculos

$$
\begin{gathered}
\left(\nu \circ R_{\lambda}\right)^{r(\gamma)}(S)=\left(\nu \circ R_{\lambda}\right)\left(\chi_{S}\right)(r(\gamma))=\int\left(R_{\lambda} \circ \chi_{S}\right)\left(\gamma^{\prime}\right) d \nu^{r(\gamma)}\left(\gamma^{\prime}\right)= \\
\iint \chi_{S}\left(\gamma^{\prime} \gamma^{\prime \prime}\right) d \lambda^{s\left(\gamma^{\prime}\right)}\left(\gamma^{\prime \prime}\right) d \nu^{s(\gamma)}\left(\gamma^{\prime}\right)=\iint \chi_{S}\left(\gamma \gamma^{\prime} \gamma^{\prime \prime}\right) d \lambda^{s\left(\gamma \gamma^{\prime}\right)}\left(\gamma^{\prime \prime}\right) d \nu^{r(\gamma)}\left(\gamma^{\prime}\right)= \\
\iint \chi_{\gamma^{-1} S}\left(\gamma^{\prime} \gamma^{\prime \prime}\right) d \lambda^{s\left(\gamma^{\prime}\right)}\left(\gamma^{\prime \prime}\right) d \nu^{s(\gamma)}\left(\gamma^{\prime}\right)=\int R_{\lambda}\left(\chi_{\gamma^{-1} S}\right)\left(\gamma^{\prime}\right) d \nu^{s(\gamma)}\left(\gamma^{\prime}\right)=
\end{gathered}
$$




$$
\left(\nu \circ R_{\lambda}\right)\left(\chi_{\gamma^{-1} S}\right)(s(\gamma))=\left(\nu \circ R_{\lambda}\right)^{s(\gamma)}\left(\gamma^{-1} S\right)
$$

o que conclui a prova de $a$ ).

b)

Como $S_{\nu}=G^{(0)}$ tem-se da última observação da proposição 3.21 que $\exists g \in \overline{\mathcal{F}}^{+}(G)$, com $\nu_{0}(g)=\chi_{G^{(0)}}$. Assim $\nu_{0} * \tilde{g}=\nu_{0}(g) \circ s=\chi_{G^{(0)}} \circ s=\chi_{G}$. Assim $\nu_{0} * \tilde{g} \nu=\left(\nu_{0} * \tilde{g}\right) \nu=\left(\chi_{G}\right) \nu=\nu$. Assim faz-se $\lambda=\tilde{g} \nu$ e tem-se o resultado.

\subsection{Medidas Transversas}

Medidas transversas são em certo sentido formas de integrar funções transversas. $\mathrm{O}$ interesse neste objeto matemático é devido ao teorema 3.28, o principal deste capítulo que fornece uma bijeção entre funções transversas definidas sobre um grupoide $G$ e um certo conjunto de medidas sobre $G^{(0)}$.

Até a abordagem do assunto de medidas transversas os trabalhos de Connes e Kastler são similares. Este texto focou mais nos desenvolvimentos realizados por Kastler pois estes são mais completos e melhor trabalhados. Porém esta seção será focada no trabalho original de Connes um vez que o formalismo de medidas transversas de Connes é mais simples do que o utilizado por Kastler e ainda assim é suficiente para esta dissertação.

Definição 3.24. Seja $G$ um grupoide, e seja $G^{(2)}$ o grupo de elementos multiplicáveis conforme definido anteriormente. Toda aplicação mensurável $\delta$ de $G$ sobre o grupo multiplicativo $\mathbb{R}_{+}^{*}$ que satisfaz a seguinte propriedade

$$
\delta\left(\gamma_{1} \gamma_{2}\right)=\delta\left(\gamma_{1}\right) \delta\left(\gamma_{2}\right), \quad \forall\left(\gamma_{1}, \gamma_{2}\right) \in G^{(2)}
$$

é denominada função modular, ou simplesmente homomorfismo .

Observa-se que uma função modular não é necessariamente um homomorfismo no sentido usual, uma vez que $G$ não é necessariamente um grupo. A ambiguidade é justificada pela analogia entre grupo e grupoide e entre função modular e homomorfismos de grupo.

Relembra-se que $\mathcal{E}^{+}$representa o espaço das funções transversas próprias sobre $G$ e $\mathcal{C}^{+}$ representa o espaço dos $G$-kernels próprios sobre $G$.

Definição 3.25. Uma medida transversa de módulo $\delta$ sobre $G$ é toda a aplicação $\Delta$ : $\mathcal{E}^{+} \rightarrow[0,+\infty]$ que é

a) Linear, i.e., $\Delta(\alpha \nu+\beta \mu)=\alpha \Delta(\nu)+\beta \Delta(\mu), \forall \alpha, \beta \in \mathbb{R}^{+}, \forall \nu, \mu \in \mathcal{E}^{+}$;

b) Normal, i.e., $\Delta\left(\sup \nu_{n}\right)=\sup \left(\Delta\left(\nu_{n}\right)\right)$ sempre que $\nu_{n} \nearrow \nu \operatorname{com} \nu \in \mathcal{E}^{+}$;

c) De módulo $\delta$, i.e., para toda a dupla $\nu, \nu^{\prime} \in \mathcal{E}^{+}$e todo kernel $\lambda$ tal que $\lambda^{y}\left(\chi_{G}\right)=$ $\lambda^{y}(1)=1, \forall y \in G^{(0)}$ tem-se que 


$$
\nu * \delta \lambda=\nu^{\prime} \Rightarrow \Delta(\nu)=\Delta\left(\nu^{\prime}\right)
$$

Se $\delta=1$ a condição $c$ exprime invariância de $\Delta$ por translação a direita sobre $G$, agindo sobre $\mathcal{E}^{+} . \Delta$ é dita semi-finita se para todo $\nu \in \mathcal{E}^{+}$tem-se que $\Delta(\nu)=\sup \left\{\Delta\left(\nu^{\prime}\right), \nu^{\prime} \leq\right.$ $\left.\nu, \Delta\left(\nu^{\prime}\right)<+\infty\right\}$ e que $\Delta$ é $\sigma$-finita se existe função fiel da forma $\nu=\sup \nu_{n}, \Delta\left(\nu_{n}\right)<$ $+\infty, \forall n \in \mathbb{N}$. Daqui em diante se supõe $\Delta$ semi-finito.

Para todo $\nu \in \mathcal{E}^{+}$tem-se que $(f \circ s) \nu \in \mathcal{E}^{+}$para todo $f \in \mathcal{F}^{+}\left(G^{(0)}\right)$, a igualdade $\Delta_{\nu}(f)=\Delta((f \circ s) \nu)$ define assim uma medida positiva $\Delta_{\nu}$ sobre $G^{(0)}$ (que é semi-finita desde que $\Delta$ seja semi-finita).

O próximo resultado, enunciado por Connes, é um lema técnico necessário mais adiante.

Lema 3.26. Se $\nu, \nu^{\prime} \in \mathcal{E}^{+}$e $\lambda \in \mathcal{C}^{+}$verificam a condição $\nu^{\prime}=\nu * \lambda$ segue que $\Delta\left(\nu^{\prime}\right)=$ $\Delta_{\nu}\left(\lambda\left(\delta^{-1}\right)\right)$.

Demonstração. Supõe-se primeiramente que $\lambda^{y} \neq 0, \forall y$, tem-se o seguinte desenvolvimento

$$
\begin{gathered}
\nu^{\prime}=\nu * \lambda=\nu * \delta \cdot \frac{1}{\delta} \lambda= \\
\nu * \delta . \delta^{-1} \frac{\lambda\left(\delta^{-1}\right) \circ r}{\lambda\left(\delta^{-1}\right) \circ r} \lambda=\nu *\left(\delta \lambda\left(\delta^{-1}\right) \circ r\right) \mu
\end{gathered}
$$

com $\mu=\frac{\lambda}{\delta\left(\lambda\left(\delta^{-1}\right) \text { or }\right)}$, nota-se que $\mu(1)=1$. Afirma-se que a seguinte igualdade é válida:

$$
\nu *\left(\left(\lambda\left(\delta^{-1}\right) \circ r\right) \delta\right) \mu=\left(\lambda\left(\delta^{-1}\right) \nu\right) * \delta \mu
$$

Segue a verificação da equação (3.9)

$$
\begin{gathered}
\left(\nu *\left(\lambda\left(\delta^{-1}\right) \circ r\right) \delta \mu\right)(g)(x)=\nu R_{\left(\lambda\left(\delta^{-1}\right) \circ r\right) \delta \mu}(g)(x)=\int R_{\left(\lambda\left(\delta^{-1}\right) \circ r\right) \delta \mu}(g)(\gamma) d \nu^{x}(\gamma)= \\
\iint g\left(\gamma \gamma^{\prime}\right)\left(\lambda\left(\delta^{-1}\right) \circ r\right)\left(\gamma^{\prime}\right) \delta\left(\gamma^{\prime}\right) d \mu^{s(\gamma)}\left(\gamma^{\prime}\right) d \nu^{x}(\gamma)
\end{gathered}
$$

mas $\left(\lambda\left(\delta^{-1}\right) \circ r\right)\left(\gamma^{\prime}\right)=\int \delta^{-1}\left(\gamma^{\prime \prime}\right) d \lambda^{r\left(\gamma^{\prime}\right)=s(\gamma)}\left(\gamma^{\prime \prime}\right)$, assim $\lambda\left(\delta^{-1} \circ r\right)\left(\gamma^{\prime}\right)$ não depende de $\gamma^{\prime}$ em $(3.10), \log O$

$$
\begin{gathered}
\iint g\left(\gamma \gamma^{\prime}\right) \delta\left(\gamma^{\prime}\right) d \mu^{s(\gamma)}\left(\gamma^{\prime}\right)\left(\lambda\left(\delta^{-1}\right) \circ r\right)(\gamma) d \nu^{x}(\gamma)= \\
\int\left(R_{\delta\left(\gamma^{\prime}\right) \mu}(g)\left(\lambda\left(\delta^{-1}\right) \circ r\right)\right)(\gamma) d \nu^{x}(\gamma)=\left(\left(\lambda\left(\delta^{-1}\right) \circ r\right) \nu * \delta \mu\right)(g)(x) .
\end{gathered}
$$

Com 3.9 verificada procede-se a demonstração

$$
\Delta\left(\nu^{\prime}\right)=\Delta\left(\lambda\left(\delta^{-1}\right) \nu * \delta \mu\right)=\Delta\left(\lambda\left(\delta^{-1}\right) \nu\right)=\Delta_{\nu}\left(\lambda\left(\delta^{-1}\right)\right) .
$$


Nota-se que a igualdade $\nu^{\prime}=\lambda\left(\delta^{-1}\right) \nu * \delta \mu$ continua válida mesmo após retirar a suposição de que $\lambda^{y} \neq 0, \forall y$, assim a equação (3.11) continua válida, donde segue a demonstração.

Lema 3.27. [3] Seja $\Delta$ uma medida transversa semi-finita de módulo $\delta$. As seguintes afirmações são válidas:

a) Para $\nu, \nu^{\prime} \in \mathcal{E}^{+}$e $f \in \mathcal{F}^{+}(G)$ tem-se que

$$
\Delta_{\nu}\left(\nu^{\prime}(\tilde{f})\right)=\Delta_{\nu^{\prime}}\left(\nu\left(\delta^{-1} f\right)\right)
$$

b) Sejam $\nu, \nu^{\prime} \in \mathcal{E}^{+}, \lambda \in \mathcal{C}^{+} \operatorname{com} \nu^{\prime}=\nu * \delta \nu$ e defina $D(f)$ por $D(f)=\lambda(f \circ s)$, com $f \in \mathcal{F}^{+}(G)$. Segue que $\Delta_{\nu^{\prime}}=\Delta_{\nu} \circ D$ e $\Delta_{\nu^{\prime}}\left(\nu^{\prime}(f)\right)=\Delta_{\nu}\left(\nu\left(L_{\lambda} R_{\delta \lambda}(f)\right)\right)$ para toda $f \in \mathcal{F}^{+}(G)$.

Demonstração. Para $f \in \mathcal{F}^{+}(G)$, aplique a igualdade $\Delta(\nu * \delta \lambda)=\Delta_{\nu}\left(\delta \lambda\left(\delta^{-1}\right)\right)=\Delta_{\nu}(\lambda(1))$ a $\lambda=\tilde{f} \nu^{\prime}$, donde

$$
\Delta\left(\nu * \delta \tilde{f} \nu^{\prime}\right)=\Delta_{\nu}\left(\tilde{f} \nu^{\prime}(1)\right)=\Delta_{\nu}\left(\nu^{\prime}(\tilde{f})\right)
$$

de 3.22 item $(i v)$ segue que $\nu * \delta \tilde{f} \nu^{\prime}=(\nu(\tilde{\delta} f) \circ s) \nu^{\prime}$, donde se obtêm a igualdade

$$
\Delta_{\nu^{\prime}}(\nu(\tilde{\delta} f))=\Delta_{\nu}\left(\nu^{\prime}(\tilde{f})\right)
$$

que prova $a$, pois $\tilde{\delta}=\delta^{-1}$.

b) Para $f \in \mathcal{F}^{+}\left(G^{(0)}\right)$ tem-se que

$$
\begin{gathered}
\Delta_{\nu^{\prime}}(f)=\Delta((f \circ s)(\nu * \delta \lambda))=\Delta(\nu *(f \circ s) \delta \lambda)= \\
\Delta_{\nu}\left((f \circ s) \delta \lambda\left(\delta^{-1}\right)\right)=\Delta_{\nu}(\lambda(f \circ s))=\Delta_{\nu}(D(f)) .
\end{gathered}
$$

Onde foram utilizadas o lema 3.26 e a igualdade $(f \circ s)(\nu * \delta \lambda)=\nu *(f \circ s) \delta \lambda$ que é facilmente verificada por calculo direto.

Para $f \in \mathcal{F}^{+}(G)$ (e não $\mathcal{F}^{+}\left(G^{(0)}\right)$ como anteriormente) tem-se que

$$
\begin{gathered}
\Delta_{\nu^{\prime}}\left(\nu^{\prime}(f)\right)=\Delta_{\nu^{\prime}}\left(\nu\left(R_{\delta \lambda}(f)\right)\right)= \\
\Delta_{\nu} \circ D\left(\nu\left(R_{\delta \lambda}(f)\right)\right)=\Delta_{\nu} \circ \lambda\left(\nu\left(R_{\delta \lambda}(f)\right) \circ s\right)
\end{gathered}
$$

Onde 3.12 foi utilizada. Mas tem-se que se $g \in \mathcal{F}^{+}(G) D(\nu(g))=\lambda(\nu(g) \circ s)=\nu(\lambda * g)$, $\log \mathrm{O}$

$$
\Delta_{\nu}\left(D\left(\nu\left(R_{\delta \lambda}(f)\right)\right)\right)=\Delta_{\nu} \circ \nu\left(L_{\lambda} R_{\delta \lambda}(f)\right)
$$


Conforme anunciado no começo desta seção o próximo resultado devido a Connes é essencial para este trabalho. Este resultado garante uma bijeção entre o conjunto de medidas transversas em um grupoide $G$ e um subconjunto especial das medidas em $G^{(0)}$.

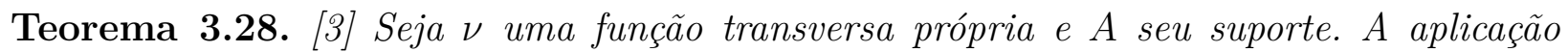
$\Delta \rightarrow \Delta_{\nu}$ é uma bijeção entre o conjunto de medidas transversas de módulo $\delta$ sobre $G_{A}$ e o conjunto das medidas positivas $\mu$ sobre $G^{(0)}$ verificando as seguintes condições equivalentes:

1) $\delta(\mu \circ \nu)^{\sim}=\mu \circ \nu$, i.e., $\mu\left(\nu\left(\tilde{f} \delta^{-1}\right)\right)=\mu(\nu(f)), \forall f \in \overline{\mathcal{F}}^{+}(G)$.

2) Se $\lambda, \lambda^{\prime} \in \mathcal{C}^{+}$são tais que $\nu * \lambda=\nu * \lambda^{\prime} \in \mathcal{E}^{+}$então $\mu\left(\delta^{-1} \lambda(1)\right)=\mu\left(\delta^{-1} \lambda^{\prime}(1)\right)$.

Demonstração. Pode-se supor que $A=G^{(0)}$. Como $\nu$ é fiel, todo $\nu^{\prime} \in \mathcal{E}^{+}$é da forma $\nu^{\prime}=\nu * \delta \lambda, \lambda \in \mathcal{C}^{+}$(3.23 item b) donde $\lambda \in \mathcal{C}^{+}$e segue que a aplicação $\Delta \mapsto \Delta_{\nu}$ é injetiva, pois suponha que $\Delta_{\nu}(\psi)=\Delta_{\nu}^{\prime}(\psi), \forall \psi$ e que $\Delta(\xi) \neq \Delta^{\prime}(\xi)$ para alguma função transversa própria $\xi$. Mas $\xi=\nu * \delta \lambda$ para algum kernel $\lambda$, logo

$$
\left.\begin{array}{l}
\Delta(\nu * \delta \lambda)=\Delta_{\nu}\left(\lambda\left(\delta^{-1}\right)\right) \\
\Delta^{\prime}(\nu * \delta \lambda)=\Delta_{\nu}\left(\lambda\left(\delta^{-1}\right)\right)
\end{array}\right\} \Rightarrow \Delta(\xi)=\Delta^{\prime}(\xi)
$$

A proposição 3.27 mostra que $\Delta_{\nu}$ verifica a condição 1 :

$$
\Delta_{\nu} \circ \nu\left(\delta^{-1} g\right)=\Delta_{\nu}\left(\nu\left(\delta^{-1}\left(\delta^{-1} g\right)^{\sim}\right)\right)=\Delta_{\nu} \circ \nu(\tilde{g})
$$

$1 \Rightarrow 2$ ) Seja $\mu$ medida verificando 1 e seja $\vartheta \in \mathcal{C}^{+}$. Para toda $f \in \mathcal{F}^{+}(G)$ tem-se que

$$
\mu(\vartheta(\nu(f) \circ s))=(\mu \circ \nu)(\vartheta * f)
$$

pois

$$
\begin{gathered}
\vartheta(\nu(f) \circ s)(x)=\int(\nu(f) \circ s)(\gamma) d \vartheta^{x}(\gamma)=\iint f\left(\gamma^{\prime}\right) d \nu^{s(\gamma)}\left(\gamma^{\prime}\right) d \vartheta^{x}(\gamma) \\
\iint f\left(\gamma \gamma^{-1} \gamma^{\prime}\right) d \nu^{s(\gamma)}\left(\gamma^{\prime}\right) d \vartheta^{x}(\gamma)=\iint f\left(\gamma^{-1} \gamma^{\prime}\right) d \nu^{r(\gamma)=x}\left(\gamma^{\prime}\right) d \vartheta^{x}(\gamma)= \\
\iint f\left(\gamma^{-1} \gamma^{\prime}\right) d \nu^{x}\left(\gamma^{\prime}\right) d \vartheta^{x}(\gamma)=\nu^{x}(\vartheta * f) .
\end{gathered}
$$

Além disso tem-se que

$$
\tilde{\delta}(\vartheta * f)^{\sim}=R_{\delta \vartheta}(\tilde{\delta} f)
$$

pois

$$
\begin{gathered}
\tilde{\delta}(\gamma) \int f\left(\gamma^{\prime-1} \gamma^{-1}\right) d \vartheta^{s(\gamma)}\left(\gamma^{\prime}\right)=\int f\left(\gamma^{\prime-1} \gamma^{-1}\right) \frac{\delta\left(\gamma^{\prime-1} \gamma^{-1}\right)}{\delta\left(\gamma^{\prime-1}\right)} d \vartheta^{s(\gamma)}\left(\gamma^{\prime}\right)= \\
\int \tilde{f}\left(\gamma \gamma^{\prime}\right) \tilde{\delta}\left(\gamma \gamma^{\prime}\right) \delta\left(\gamma^{\prime}\right) d \vartheta^{s(\gamma)}\left(\gamma^{\prime}\right)=R_{\delta \vartheta}(\tilde{f} \delta)
\end{gathered}
$$


Aplicando (3.13) e (3.14) e a hipótese segue que

$$
\begin{gathered}
\mu(\vartheta(\nu(f) \circ s))=(\mu \circ \nu)(\vartheta * f)=\delta(\mu \circ \nu)^{\sim}(\vartheta * f)= \\
(\mu \circ \nu)\left(\tilde{\delta}(\vartheta * f)^{\sim}\right)=(\mu \circ \nu)\left(R_{\delta \vartheta}(\tilde{\delta} f)\right)
\end{gathered}
$$

Assim define-se $\vartheta=\frac{1}{\delta} \lambda$ e $\vartheta^{\prime}=\frac{1}{\delta} \lambda^{\prime}$, logo substituindo em (3.15) tem-se que

$$
\mu(\vartheta(\nu(f) \circ s))=\mu((\nu * \lambda)(\tilde{\delta f}))=\mu\left(\left(\nu * \lambda^{\prime}\right)(\tilde{\delta f})\right)=\mu\left(\vartheta^{\prime}(\nu(f) \circ s)\right)
$$

Sabe-se da proposição 3.21 que existe função $f$ tal que $\nu(f) \circ s=1$, substituindo esta $f$ em (3.16) tem-se que

$$
\mu\left(\lambda\left(\delta^{-1}\right)\right)=\mu(\vartheta(1))=\mu\left(\vartheta^{\prime}(1)\right)=\mu\left(\lambda^{\prime}\left(\delta^{-1}\right)\right)
$$

Donde se obtêm 2 .

Mostra-se a existência de $\Delta$ a partir de $\mu$. Primeiramente tem-se que todo $\nu^{\prime} \in \mathcal{E}^{+}$é da forma $\nu^{\prime}=\nu * \lambda$, a condição 2 permite definir $\Delta$ pela igualdade

$$
\Delta(\nu * \lambda)=\mu\left(\delta^{-1} \lambda(1)\right)
$$

Verifica-se as condições $a, b, c$ da definição 1:

a) Trivial.

b) Para $\nu_{n}^{\prime} \nearrow \nu_{\infty}^{\prime}$ sobre $\mathcal{E}^{+}$e $f \in \mathcal{F}^{+}(G)$ tal que $\nu(\tilde{f})=1$ (proposição 3.23) e tem-se que $\nu_{n}^{\prime}=\nu * \delta \lambda_{n}$ donde $\lambda_{n}=\delta^{-1} f \nu_{n}^{\prime}$ (proposição 3.22) e verifica-se que

$$
\nu * \delta \lambda_{n}=\nu *\left(\delta^{-1} f \nu_{n}^{\prime}\right)=(\nu * f) \nu_{n}^{\prime}=\nu_{n}^{\prime}
$$

Assim

$$
\Delta\left(\nu_{n}^{\prime}\right)=\Delta\left(\nu * \delta \lambda_{n}\right)=\mu\left(\delta^{-1} \delta \lambda_{n}(1)\right)=\mu\left(\nu_{n}^{\prime}\left(\delta^{-1} f\right)\right) .
$$

Como $\nu_{n}^{\prime} \nearrow \nu_{\infty}^{\prime}$ a sequência crescente $\nu_{n}^{\prime}\left(f \delta^{-1}\right)$ de funções mensuráveis sobre $G^{(0)}$ que convergem a função $\nu_{\infty}^{\prime}\left(f \delta^{-1}\right)$ de modo que $\mu\left(\nu_{n}^{\prime}\left(\delta^{-1} f\right)\right) \nearrow \mu\left(\nu_{\infty}^{\prime}\left(\delta^{-1} f\right)\right)$. Assim de (3.17) segue que $\Delta\left(\nu_{n}^{\prime}\right) \nearrow \Delta\left(\nu_{\infty}^{\prime}\right)$.

c) Sejam $\nu_{1}=\nu * \delta \lambda_{1} \in \mathcal{E}^{+}$e $\lambda \in \mathcal{C}^{+}$com $\nu_{2}=\nu_{1} * \delta \lambda \in \mathcal{E}^{+}$. Segue que $\nu_{2}=\left(\nu * \delta \lambda_{1}\right) * \delta \lambda$ $=\nu * \delta\left(\lambda_{1} * \lambda\right)$ donde $\Delta\left(\nu_{2}\right)=\mu\left(\left(\lambda_{1} * \lambda\right)(1)=\mu\left(\lambda_{1}(1)\right)\right.$ com $\left(\lambda_{1} * \lambda\right)(1)=\lambda_{1}(1)$ donde segue o resultado.

Resta verificar que $\Delta_{\nu}=\mu$, seja $f \in \mathcal{F}^{+}\left(G^{(0)}\right)$ e seja $\lambda \in \mathcal{C}^{+}$tal que $\lambda^{y}=f(y) \epsilon_{y}, \forall y \in$ $G^{(0)}$, segue que $\delta \lambda=\lambda$,

$$
\Delta_{\nu}(f)=\Delta((f \circ s) \nu)=\Delta(\nu * \delta \lambda)=\mu(\lambda(1))=\mu(f)
$$




\subsection{Exemplos}

Nesta seção são abertos os exemplos apresentados por Kastler em seu artigo. Os exemplos tiveram de ser adaptados a este texto, uma vez que o formalismo de medidas transversas do referido autor é diferente do utilizado neste capítulo.

Exemplo 3.29. [7] Grupo. Um grupoide de medida é um grupo sse $G^{(0)}=\{e\}$. Os $G$-kernels são nesse caso medidas positivas em $G$, com a convolução de $G$-kernels sendo a convolução usual de medidas em um grupo. As funções transversas são medidas invariantes a esquerda

$$
\nu^{r(\gamma)}=\gamma \nu^{s(\gamma)}
$$

e portanto se $\mathrm{G}$ for um grupo localmente compacto as funções transversas são medidas de Haar invariantes a esquerda donde segue que são únicas a menos de uma constante multiplicativa [4] p. 344.

A convolução * é igual a convolução usual de funções em um grupo. O homomorfismo $\delta$ é simplesmente a função modular no grupo (que é constante para toda a função transversa, uma vez que elas são múltiplos). Observe que todas a medidas em $\{e\}$ são únicas a menos de uma constante. Seja $M$ medida em $\{e\}$. Segue diretamente de um resultado sobre medidas de Haar (proposição 11.14 [4]) que a seguinte igualdade é satisfeita

$$
M\left(\nu_{0}\left(\delta^{-1} \tilde{f}\right)\right)=M\left(\nu_{0}(f)\right), \forall f \in \overline{\mathcal{F}}^{+}(G)
$$

Fixe $\delta$, tem-se pelo teorema 3.28 que se $\nu$ é função transversa própria ( $\nu$ é função transversa e $\left.\nu^{e}(G)<\infty\right)$ então segue que existe bijeção entre as medidas transversas em $G$ e as medidas $M$ em $\{e\}, \Delta \mapsto \Delta_{\nu}$, mas $\Delta_{\nu}$ é única a menos de uma constante multiplicativa, donde segue que $\exists ! \Delta$ tal que $\Delta_{\nu}$ é medida de probabilidade.

Exemplo 3.30. [7] Espaço. Se $G=G^{(0)}=X$, i.e., todos os elementos de $G$ são idempotentes, os $G$-kernels $\delta$ coincidem com as funções transversas $f$ em $X$ (uma vez que $\nu^{r(\gamma)}=\gamma \nu^{s(\gamma)}$ é trivialmente satisfeita). Além disso tem-se uma relação de um para um entre as funções $f$ em $X$ com os $G$-kernels da seguinte forma

$$
\lambda^{x}=f \varepsilon_{x}
$$

$\operatorname{com} \varepsilon_{x}$ medida de Dirac com suporte em $x \in X$, i.e.,

$$
\lambda(g)(x)=\int g(\gamma) d \lambda^{x}(\gamma)=\int g\left(I d_{x}\right) d \lambda^{x}(\gamma)=g\left(I d_{x}\right) \cdot f(x)
$$

Nota-se que a convolução coincide com a multiplicação usual

$$
(h \underset{\nu}{*} g)(\gamma)=\int h\left(\gamma^{\prime}\right) g\left(\gamma^{\prime-1} \gamma\right) d \nu^{s(\gamma)}\left(\gamma^{\prime}\right)=h\left(I d_{s(\gamma)}\right) g\left(I d_{s(\gamma)}\right) f(s(\gamma))
$$


O homomorfismo $\delta$ é unitário, uma vez que só é avaliado nos elementos identidades e $\tilde{f}=f, \forall f \in \overline{\mathcal{F}}^{+}(G)$ logo a condição $M\left(\nu_{0}\left(\delta^{-1} \tilde{f}\right)\right)=M\left(\nu_{0}(f)\right)$ é trivialmente satisfeita para toda função transversa $\nu_{0}$ e medida $M$. Assim o teorema 3.28 garante que existe uma bijeção entre as medidas transversas e todas as medidas de $X$.

Exemplo 3.31. [7] Espaço $\times$ Espaço. Se o grupoide $G$ é induzido pela relação de equivalência trivial em que todo os elementos pertencem a mesma classe de equivalência, i.e., $x \sim y, \forall x, y \in G^{(0)}$. Tem-se neste caso que $G=X \times X$, onde $X=G^{(0)}$. Tem-se que $r(y, x)=y, s(y, x)=x,(y, x)^{-1}=(x, y)$ e $(y, x) .(x, t)=(y, t), x, y, t \in X$.

$\mathrm{O}$ símbolo $\otimes$ deve ser entendido como o produto tensorial e age da seguinte maneira $\alpha \otimes \beta(x, y)=\alpha(x) . \beta(y)$. Tem-se que existe uma bijeção entre $G$-kernels $\lambda$ com kernels $\underline{\lambda}: X \rightarrow X$, dada por $\lambda^{y}=\varepsilon_{y} \otimes \underline{\lambda_{y}}, y \in X$. Pois se $\underline{\lambda_{y}}$ é kernel então

$$
\left(\varepsilon_{y} \otimes \underline{\lambda_{y}}\right)(f)=\int f(t, x) d\left(\varepsilon_{y} \otimes \underline{\lambda_{y}}\right)(t, x)=\int f(y, x) d \underline{\lambda_{y}}(x)
$$

que é função mensurável. Por outro lado se $\lambda^{y}$ é kernel então

$$
\left(\lambda^{y}\right)(f)=\int f(t, x) d \lambda^{y}(t, x)=\int f(y, x) d \lambda^{y}(y, x)=\int f(y, x) d \underline{\lambda_{y}}(x)
$$

que é mensurável.

A convolução de $G$-kernels $\lambda$ é obtida do produto dos correspondentes kernels $\underline{\lambda}: \underline{\lambda * \mu}=$ $\underline{\lambda} \circ \underline{\mu}$

$$
\begin{gathered}
\lambda * \mu(f)(y)=\lambda \circ R_{\mu}(f)(y)=\int R_{\mu}(f)(t, x) d \lambda^{y}((t, x))= \\
\iint f((y, x)(x, w)) d \mu^{x}((x, w)) d \lambda^{y}((y, x))=\iint f(y, w) d \underline{\mu_{x}}(w) d \underline{\lambda_{y}}(x)= \\
\left(\underline{\lambda} \circ R_{\underline{\mu}}\right)(f)
\end{gathered}
$$

Observa-se que $\lambda$ é $\sigma$-finito (próprio) sse $\underline{\lambda}$ é $\sigma$-finito (próprio). Além disso para uma função transversa $\nu$ tem-se que $\underline{\nu_{y}}=\underline{\nu}$ (independente de y) pois

$$
\begin{gathered}
\nu^{y}(A)=\int \chi_{A}(y, x) d \underline{\nu_{y}}(x) \\
\nu^{x}((x, y) . A)=\int \chi_{(x, y) \cdot A}(x, t) d \underline{\nu_{x}}(t)=\int \chi_{A}(y, t) d \underline{\nu_{x}}(t)
\end{gathered}
$$

Como $\nu^{y}(A)=\nu^{x}((x, y) . A), \forall A \log \underline{\nu_{y}}=\underline{\nu_{x}}, \forall x, y$. Assim as funções transversas consistem de medidas positivas $\underline{\nu}$ em $X$, com $\nu$ fiel sse $\underline{\nu} \neq 0$. A convolução de funções é dada por 


$$
(f \underset{\nu}{*} g)(y, x)=\int f((t, z)) g\left((t, z)^{-1} \cdot(y, x)\right) d \nu^{y}(t, z)=\int f(y, z) \cdot g((z, x)) d \underline{\nu_{y}}(z)
$$

As funções modulares são da forma $\delta(x, y)=e^{-V(y)} \cdot e^{+V(x)}$, onde $V$ é função mensurável em $X . \operatorname{Assim} \nu\left(\delta^{-1}\right)=\underline{\nu}\left(e^{V}\right) e^{-V}$, pois

$$
\begin{gathered}
\nu(\delta)(y)=\int \delta(t, z) d \nu^{y}(t, z)=\int \delta(y, z) \underline{d \underline{\nu_{y}}}(z)= \\
\int e^{-V(y)} \cdot e^{V(z)} d \underline{\nu_{y}}(z)=e^{-V} \underline{\nu}\left(e^{V}\right) .
\end{gathered}
$$

Afirmação: Existe a menos de uma constante uma única medida transversa. Segue a prova deste fato. Defina $\nu_{a}$ por $\underline{\nu_{a}}=\varepsilon_{a}, a \in X$, tem-se que $\nu_{a} * \nu=\nu, \forall \nu \in \mathcal{E}^{*}$, pois

$$
\begin{gathered}
\left(\nu_{a} * \nu\right)(f)(y)=\left(\nu_{a} \circ R_{\nu}\right)(f)(y)=\int R_{\nu}(f)(t, z) d \nu_{a}^{y}(t)= \\
\iint f((t, z) \cdot(w, x)) d \nu^{z}(w, x) d \nu_{a}^{y}(t)=\int f((y, a) \cdot(a, x)) d \underline{\nu}(x)= \\
\int f(y, x) d \underline{\nu}(x)=\nu(f)(y) .
\end{gathered}
$$

Assim tem-se que se $\Delta$ é medida transversa então

$$
\begin{gathered}
\Delta(\nu)=\Delta\left(\nu_{a} * \nu\right)=\Delta_{\nu_{a}}\left(\nu\left(\delta^{-1}\right)\right)=\Delta_{\nu_{a}}\left(e^{-V} \underline{\nu}\left(e^{V}\right)\right)= \\
\underline{\nu}\left(e^{V}\right) \Delta_{\nu_{a}}\left(e^{-V}\right)=\underline{\nu}\left(e^{V}\right) . k
\end{gathered}
$$

$\operatorname{com} k=\Delta_{\nu_{a}}\left(e^{-V}\right)$ uma constante.

Exemplo 3.32. [7] Grupoide da Ação de Um Grupo. Seja $\Gamma \times X \ni(g, X) \rightarrow g x \in X$ a ação mensurável de um grupo mensurável $\Gamma$ em um espaço localmente compacto $X, G=X \times \Gamma$ é dada a estrutura de um grupoide mensurável como segue:

- $G^{(0)}=X$,

- $r(y, g)=y$,

- $s(y, g)=g^{-1} y$

- $(y, g)^{-1}=\left(g^{-1} y, g^{-1}\right)$

- $(y, g)\left(g^{-1} y, h\right)=(y, g h)$

Os $G$-kernels $\lambda$ tem uma relação de 1 a 1 com os kernels $\bar{\lambda}: \Gamma \rightarrow X$, que é tornada explicita pela relação

$$
\lambda^{y}=\varepsilon_{y} \otimes \bar{\lambda}_{y}
$$


pois

$$
\begin{gathered}
\lambda^{y}(f)=\int f(\gamma) d \lambda^{y}(\gamma)=\int f((x, g)) d \lambda^{y}(x, g)= \\
\int f(y, g) d \lambda^{y}(y, g)=\int f(y, g) d \bar{\lambda}_{y}(g) .
\end{gathered}
$$

A $\sigma$-finitude de $\lambda$ corresponde a $\sigma$-finitude de $\bar{\lambda}$. Portanto as funções transversas correspondem aos kernels $\sigma$-finitos $\bar{\nu}_{s x}=s \bar{\nu}_{x}, x \in X, s \in G$.

$$
\begin{gathered}
\nu^{r(\gamma)}=\gamma \nu^{s(\gamma)} \\
\nu^{s x}=(s x, s) \nu^{x} \Rightarrow \bar{\nu}_{s x}=s \bar{\nu}_{x}
\end{gathered}
$$

$x \in X, s \in G$. A convolução de funções é dada por

$$
\begin{gathered}
(\underset{\nu}{*} g)(y, s)=\int f((x, t)) g\left((x, t)^{-1}(y, s)\right) d \nu^{r(y, s)=y}((x, t))= \\
\int f((y, t)) g\left(\left(t^{-1} y, t^{-1}\right) \cdot(y, s)\right) d \bar{\nu}_{y}(t)=\int f(y, t) \cdot g\left(t^{-1} y, t^{-1} s\right) d \bar{\nu}_{y}(t) .
\end{gathered}
$$

Se $G$ é localmente compacto com medida de Haar a esquerda $\sigma$, obtem-se que $\nu_{0} \in \mathcal{E}^{+}$ ao fazer $(\bar{\nu})_{y}=\sigma, y \in X$.

Segue diretamente da definição de funções modulares que estas são caracterizadas pela seguinte condição,

$$
\delta(y, s t)=\delta(y, s) \delta\left(s^{-1} y, t\right), y \in X, s, t \in G .
$$

As medidas transversas são caracterizadas como segue para um grupo localmente compacto $G$ : uma medida positiva $M$ em $X$ é da forma $M=\Delta_{\sigma}$ sse satisfaz

$$
d M\left(s^{-1} x\right)=\Delta(s) \delta\left(x, s^{-1}\right) d M(x), x \in X, s \in G
$$

com $\Delta$ função modular em $G$ (esta função modular é referente a medida de Haar [4]). A relação (3.18) é justificada pelo seguinte raciocínio, se $m=\Delta_{\nu_{0}} \circ \nu_{0}$ tem-se realmente para $f \in \mathcal{F}^{+}(X \times G)$

$$
\begin{gathered}
m(f)=\int f(x, g) d M(x) d \sigma(g)=m\left(\delta^{-1} \tilde{f}\right)= \\
\int \delta^{-1}(g x, g) f\left(x, g^{-1}\right) d M(g x) d \sigma(g)=\int \delta(x, g) \Delta(g)^{-1} f(x, g) d M\left(g^{-1} x\right) d \sigma(g)
\end{gathered}
$$

Exemplo 3.33. [7] Gráfico de Relação de Equivalência. Procede-se a análise do caso em que o grupoide mensurável $G$ é um grupoide compatível associado a uma relação de equivalência $\sim$ definida sobre $X$. Assume-se que $X$ é um espaço standart mensurável, donde segue que $G$ é standart, também assume-se que a diagonal em $X \times X$ é mensurável. Dado um $G$-kernel $\sigma$-finito $\lambda$, define-se o kernel $\underline{\lambda}: X \rightarrow X$ por 


$$
\underline{\lambda}(\psi)=\lambda(\psi \circ s), \psi \in \overline{\mathcal{F}}^{+}(X)
$$

assim se obtem uma bijeção $\lambda \mapsto \underline{\lambda}$ sobre kernels $\underline{\lambda}: X \rightarrow X$ tal que $\underline{\lambda_{y}}, y \in X$ é carregado pela classe de equivalência de $y$, com a inversa dada por $\lambda^{y}=\left.\left(\varepsilon_{y} \otimes \underline{\lambda_{y}}\right)\right|_{G}$. Então $\nu=$ $\left.(\varepsilon \otimes \underline{\nu})\right|_{G}$ é uma função transversa em $G \Leftrightarrow \underline{\nu_{y}}=\underline{\nu_{x}}$ sempre que $\pi(y)=\overline{\pi(x)}$. Estas funções transversas tem relação bijetiva com os kernels $\underset{\sim}{\nu}: X \rightarrow X / \sim$ fibrados por $\pi$ com a bijeção $\nu_{x} \mapsto \nu_{\pi(x)}, x \in X$, i.e., para cada $f \in \overline{\mathcal{F}}^{+}(X)$ e cada $g \in \overline{\mathcal{F}}^{+}(X \backslash \sim)$ é válida a igualdade $\nu(f . g \circ \pi)=g \cdot \nu(f)$.

O caso mais simples de relação de equivalência é quando $X / \sim$ é espaço standart, neste caso segue que existem kernels $\sigma: X \rightarrow X / \sim \operatorname{com} \sigma$ limitado e não nulo para todo $p \in X / \sim$ $\left(\left\{\sigma_{q}\right\}_{q \in X / \sim}\right.$ é uma desintegração). 


\section{Capítulo 4}

\section{Teoria de Integração não Comutativa e Siste- mas Dinâmicos}

Os sistemas dinâmicos considerados nesta seção são da forma $(M, f, \mu) \operatorname{com} M$ uma variedade riemanniana $C^{2}, f$ é um difeomorfismo $C^{2}$ e $\mu$ é uma medida de probabilidade invariante por $f$. Neste capítulo primeiramente será definido um grupoide, chamado de grupoide instável, associado a partição de $M$ em variedades instáveis.

O objetivo deste capítulo é utilizar o teorema 3.28 para exibir uma injeção entre as medidas transversas definidas em um "grupoide quase igual" ao grupoide instável e as medidas SRB do sistema $(M, f)$. Para isso será necessário definir o "grupoide quase igual". Além disso é necessário definir as funções transversas e a função modular de tal sorte que a relação $(i)$ do teorema 3.23 seja satisfeita para toda medida $\operatorname{SRB}$ do sistema $(M, f)$.

Definição 4.1. O grupoide instável (em inglês unstable grupoid) $G_{u}$ do sistema dinâmico $(M, f, \mu)$ é o grupoide principal associado a relação de equivalência induzida pelas variedades instáveis globais, $\left(\left\{W^{u}(x)\right\}_{x \in M}\right.$ conforme definida em 2.4) em $M$.

Assim o grupoide instável é obtido através da relação de equivalência: se $x, y \in M$ tem-se

$$
x \sim y \Leftrightarrow y \in W^{u}(x)=\left\{t \in M \mid \limsup _{n \rightarrow \infty} \frac{1}{n} \ln \left(d\left(f^{-n}(x), f^{-n}(t)\right)\right)<0\right\}
$$

é simples mostrar que $G_{u}$ é um grupoide compatível, i.e., $G_{u} \subset M \times M$ é mensurável [16].

Definição 4.2. Dado um sistema dinâmico $(M, f)$ e uma partição $\eta$ diz-se que $\eta$ é uma partição crescente para o automorfismo $f$ se $f \eta \preceq \eta$, i.e., se a aplicação de $f$ sobre $\eta$ resulta em uma partição $f \eta$ menos refinada do que $\eta$.

A seguinte proposição devida a Ledrappier e Strelcyn tem papel fundamental neste capítulo. Esta proposição é apresentada na forma reescrita por [16] para atender os propósitos deste texto.

Proposição 4.3. (Citado em [16], provado em [9].) Para cada probabilidade boreliana finvariante $\mu$ em $M$, existe uma partição mensurável $\eta$ de $M$ subordinada a variedade instável (definição 2.22), e um conjunto de Borel H de medida total, tal que: 
(i) Para todo $x \in H$,

$$
\bigcup_{n=-\infty}^{\infty} f^{n}\left(\eta\left(f^{-n} x\right)\right)=W^{u}(x)
$$

(ii) $\eta$ é uma partição crescente.

Deste ponto em diante será fixado um conjunto $H$ e uma partição $\eta$ da proposição 4.3.

Definição 4.4. Define-se a sequência $\left\{\eta_{n}, n \in \mathbb{Z}\right\}$ de partições mensuráveis de $M$ como segue; $\eta_{0}=\eta$; para $n \neq 0$, a partição é definida pela relação $y \in \eta_{n}(x)$ sse $y \in f^{n}\left(\eta\left(f^{-n}(x)\right)\right.$ ). Cada partição $\eta_{n}$ induz uma relação de equivalência que por sua vez induz um grupoide associado, assim denota-se $G_{n}$ o grupoide associado a partição $\eta_{n}$.

Observa-se que pela proposição 3.11 os grupoides $G_{n}$ são grupoides de medida. Além disso tem-se que para cada $n \in \mathbb{N}, G_{n}$ é grupoide compatível, i.e., $G_{n} \subset M \times M$ é subconjunto mensurável. Este fato é verdadeiro pois $M \times M$ é espaço standart e $G_{n} \subset M \times M$ é o gráfico de uma relação de equivalência induzida por uma partição mensurável é standart. E todo o subespaço standart de um espaço standart é subconjunto mensurável.

Define-se $G_{\infty}=\cup_{n \in \mathbb{N}} G_{n}$.

\subsection{Funções Transversas de Riemann}

Seja $(M, g)$ variedade riemanniana compacta, $f: M \rightarrow M$ um difeomorfismo $C^{2}$. Para $x \in M$, a inclusão $i: W^{u}(x) \rightarrow M$ induz a métrica riemanniana $g \circ i$, também denotada por $i^{*} g$, em $W^{u}(x)$. Nota-se que $W^{u}\left(f^{n}(x)\right)=f^{n}\left(W^{u}(x)\right)$. Denota-se $\beta_{x}$ a medida em $W^{u}(x)$ definida pelo elemento de volume de $g \circ i$.

Para cada $x \in M$ tem-se o seguinte isomorfismo mensurável.

$$
\begin{aligned}
s_{x}: r^{-1}(x)=\{x\} \times W^{u}(x) & \rightarrow W^{u}(x) \\
(x, y) & \mapsto y
\end{aligned}
$$

Este isomorfismo mensurável permite definir a medida $\nu_{u}^{x}$ em $r^{-1}(x) \subset G_{u}$ por

$$
\nu_{u}^{x}=\beta_{x} \circ s_{x}
$$

Seja $G \subset G_{u}$ um subgrupoide (obviamente principal). Distingue-se os mapas alvo de ambos os grupoides escrevendo-se

$$
r_{u}: G_{u} \rightarrow G_{u}^{(0)}=M \quad \text { e } \quad r: G \rightarrow G^{(0)} \subset M
$$

Para cada $x \in G^{(0)}$, as medidas $\nu_{u}^{x}$ em $r_{u}^{-1}(x)$ induzem medidas $\nu^{x}$ em $r^{-1}(x)$ pelo pullback do mapa de inclusão, i.e., $\nu^{x}(A)=\nu_{u}^{x}\left(A \cap r^{-1}(x)\right)$ para todo conjunto mensurável A.

O próximo resultado é o principal teorema da seção. Este teorema fornece uma função transversa no grupoide $G_{u, K}$. Com $K$ um conjunto de medida total e $G_{u, K}$ subgrupoide de $G_{u}$ conforme definido na equação (3.1). 
Teorema 4.5. Existe subconjunto de Borel $K \subset H \subset M$ de medida $\mu$ total tal que a coleção de medidas $\left\{\nu^{x}\right\}_{x \in K}$ induzidas pelas medidas $\nu_{u}^{x}$ como acima definem uma função transversa própria no grupoide mensurável $G_{u, K}$.

A demonstração será realizada ao final desta seção. Outro resultado também fundamental neste desenvolvimento devido a Ledrappier e Strelcyn é enunciado a seguir.

Proposição 4.6. ( Citado por [16], provado em [9].) A partição $\eta$ da proposição 4.3 pode ser escolhida de modo a satisfazer a seguinte condição: para todo conjunto de Borel $A \subset M$ a função

$$
\psi_{A}(x)=\beta_{x}(\eta(x) \cap A)
$$

é mensurável e finita em um conjunto $K^{\prime} \subset M$ mensurável de medida unitária (total).

Suporemos fixados os conjuntos $K^{\prime}, H$ e a partição $\eta$. Define-se então o conjunto $K$ por

$$
K=\bigcap_{n \in \mathbb{Z}} f^{n}\left(K^{\prime} \cap H\right) \subset M
$$

note que K é mensurável e de medida total.

Na sequência prova-se que as medidas $\nu_{0}^{x}$ induzidas nas fibras de $G_{0}$ pelas medidas $\nu_{u}^{x}$ definem uma função transversa em $G_{0}$. Novamente $G_{0}$ é o subgrupoide principal associado a partição mensurável $\eta_{0}$ de $M$. $G_{0}$ é o subgrupoide de $G_{u}$.

Proposição 4.7. [16] A coleção de medidas $\left\{\nu_{0}^{x}\right\}_{x \in M}$ define uma função transversa $\nu_{0}$ em $G_{0}$.

Demonstração. Primeiramente mostra-se que $\nu_{0}: G_{0} \rightarrow M$ é um kernel, i.e., $\forall h \in \overline{\mathcal{F}}^{+}\left(G_{0}\right)$ a função

$$
\begin{aligned}
\nu_{0}(h): M & \rightarrow \overline{\mathbb{R}}^{+} \\
y & \rightarrow \nu_{0}^{y}(h)
\end{aligned}
$$

é mensurável. Para isso seja $A \subset G_{0} \subset M \times M$ mensurável, nota-se que

$$
A \cap r^{-1}(x)=A \cap\left(\{x\} \times \eta_{0}(x)\right)=\left(\{x\} \times A^{\prime}\right) \cap\left(\{x\} \times \eta_{0}(x)\right)=\{x\} \times\left(A^{\prime} \cap \eta_{0}(x)\right)
$$

onde $A^{\prime} \subset M$ é mensurável.

A seguinte observação foi utilizada: $A \cap(\{x\} \times M)=\{x\} \cap A^{\prime}$ com $A^{\prime}$ mensurável. $A^{\prime}$ é mensurável devido a dois argumentos: primeiramente a interseção de mensuráveis é mensurável; e relembrando-se o lema 3.10 é fácil ver que a $\sigma$-álgebra induzida sobre $\{x\} \times M$ pela inclusão $\{x\} \times M \subset M \times M$ é identificável com a $\sigma$-álgebra em $M$.

Assim

$$
\begin{gathered}
\nu_{0}^{x}(A)=\nu_{u}^{x}\left(A \cap r_{0}^{-1}(x)\right)=\nu_{u}^{x}\left(\{x\} \times\left(A^{\prime} \cap \eta_{0}(x)\right)\right)= \\
\beta_{x}\left(s_{x}\left(\{x\} \times\left(A^{\prime} \cap \eta_{0}(x)\right)\right)\right)=\beta_{x}\left(A^{\prime} \cap \eta_{0}(x)\right)
\end{gathered}
$$


que é mensurável $\forall x \in G^{(0)}$ pela proposição 4.6. Define-se $\nu_{0}: G \rightarrow M$ por

$$
\begin{aligned}
\nu_{0}: \overline{\mathcal{F}}^{+}\left(G_{0}\right) & \rightarrow \overline{\mathcal{F}}^{+}(M) \\
n & \rightarrow \int h(\gamma) d \nu_{0}^{x}(\gamma)
\end{aligned}
$$

tem-se portanto que $\nu_{0}$ é afim e normal e do resultado provado segue que $\nu_{0}(h) \in \overline{\mathcal{F}}^{+}(M), \forall h \in$ $\overline{\mathcal{F}}^{+}\left(G_{0}\right)$.

Segue da definição que $\nu_{0}$ é fibrado por $r_{0}$, e como cada $x \in M$, $\nu_{0}^{x}$ é suportado em $r_{0}^{-1}(x)$. Logo $\nu_{0}$ é um $G_{0}$-kernel. A invariância de $\nu_{0}$ sob ação de $G_{0}$ é clara da construção, para $y \in \eta_{0}(x)$ tem-se que $\beta_{x}=\beta_{y}$ e que $s_{x}$ pode ser identificado com $s_{y}$, logo seja $\gamma: y \rightarrow x$ tem-se que

$$
\nu_{0}^{y}\left(\gamma^{-1}(A)\right)=\beta_{x}\left(s_{x}\left(\gamma^{-1}(A)\right)\right)=\beta_{x}\left(s_{x}(A)\right)=\nu_{0}^{x}(A)
$$

uma vez que $s_{x}$ "vê" apenas a fonte.

Seja $J^{u}(x)$ a taxa de expansão da variedade instável no ponto $x$ relativa a métrica Riemanniana, i.e.,

$$
J^{u}(x)=\left|\operatorname{Det}\left(\left.D f\right|_{E^{u}}\right)\right|
$$

Conforme definição 2.21. Nota-se que $J^{u}$ é mensurável pois $E^{u}$ é mensurável e o mapa da derivada $D f$ depende continuamente de $x \in M$.

Lema 4.8. [16] Se $\eta$ é escolhido de forma a satisfazer as condições da proposição 4.3 e proposição 4.6, então para cada número inteiro n, a partição $\eta_{n}$ também satisfaz as condições das proposições 4.3 e 4.6 .

Demonstração. Primeiramente nota-se que $\eta_{n}$ satisfaz as condições da proposição 4.3 trivialmente.

Por hipótese $\eta_{0}$ satisfaz as condições da proposição 4.6. Este fato pode ser entendido da seguinte maneira. Define-se $b: \overline{\mathcal{F}}^{+}(M) \rightarrow \overline{\mathcal{F}}^{+}(M / \eta)$ de tal forma que para $g \in \overline{\mathcal{F}}^{+}(M)$ e para cada elemento $C \in \eta$ tem-se

$$
[b(g)](C)=\beta_{x}\left(\chi_{\eta(x)} \cdot g\right)
$$

com $x \in C$ arbitrário. De 4.6 segue que $\forall A \subset M$ mensurável a função $b\left(\chi_{A}\right): M / \eta \rightarrow \overline{\mathbb{R}}^{+}$é mensurável, e quase sempre finita com respeito a medida induzida em $M / \eta$ pela medida em $M$.

Assim segue que para 4.6 ser válida para a partição $\eta$ é equivalente a afirmação de que $b: \overline{\mathcal{F}}^{+}(M) \rightarrow \overline{\mathcal{F}}^{+}(M / \eta)$ é um kernel com a hipótese adicional da finitude. Utilizando este raciocínio para as demais partições $\eta_{n}$, define-se as funções $b_{n}: \overline{\mathcal{F}}^{+}(M) \rightarrow \overline{\mathcal{F}}^{+}\left(M / \eta_{n}\right)$ por

$$
\left[b_{n}(g)\right](C)=\beta_{x}\left(\chi_{\eta_{n}(x)} \cdot g\right)
$$


Assim para mostrar que as partições $\eta_{n}$ satisfazem 4.6 basta mostrar que $b_{n}$ é kernel para todo $n$ inteiro e que satisfaça a hipótese da finitude. Com isto provado segue que $\eta_{n}$ satisfaz as condições da proposição 4.6.

A prova é realizada por indução. Mostra-se que se $b_{n}$ é kernel logo $b_{n+1}$ é kernel. Portanto supõe-se que $b_{n}$ é kernel próprio. Utiliza-se a notação $f^{*} g=g \circ f$ para todo $g \in \mathcal{F}^{+}(M)$ e $f_{*} \beta_{x}(g)=\beta_{x}\left(f^{*} g\right)=\beta_{x}(g \circ f)$ tem-se

$$
f_{*} \beta_{x}=\frac{1}{f^{-1 * J^{u}}} \beta_{f(x)}=\theta \beta_{f(x)}
$$

A equação (4.1) é uma consequência da fórmula de mudança de variável sob sinal de integração, segue a obtenção da equação

$$
\begin{gathered}
\frac{1}{f^{-1 *} J^{u}} \beta_{f(x)}(W)=\int_{f(\Omega)}\left(W \cdot \frac{1}{J^{u} \circ f^{-1}}\right)(x) d x=\int_{f(\Omega)} w(x) d x= \\
\int_{\Omega} w \circ f(x)\left|\operatorname{det} D_{x} f\right| d x=\int_{\Omega}\left(w \cdot \operatorname{det} D_{x} f \circ f^{-1}\right) \circ f(x) d x= \\
\int_{\Omega} W \circ f(x) d x=f_{*} \beta_{x}(W)
\end{gathered}
$$

Onde $W \in \overline{\mathcal{F}}^{+}(M)$ é uma função real integrável, $w=W \cdot \frac{1}{J^{u} \circ f^{-1}}$ também integrável, e $\Omega$ é um domínio de integração. Nota-se que $J^{u}>0, \operatorname{logo} \theta$ é finita. Também tem-se que

$$
f^{*}\left[\chi_{\eta_{n+1}(f(x))}\right]=\chi_{\eta_{n}(x)}
$$

uma vez que

$$
f(y) \in f^{n+1}\left[\eta\left[f^{-n-1}(f(x))\right]\right] \Leftrightarrow y \in f^{n}\left[\eta\left(f^{-n}(x)\right)\right] \Leftrightarrow y \in \eta_{n}(x)
$$

De (4.1) e (4.2) segue o desenvolvimento

$$
\begin{gathered}
\beta_{x}\left(\chi_{\eta_{n}(x)} . g\right)=\beta_{x}\left(f^{*}\left[\chi_{\eta_{n+1}(f(x))}\right] \cdot f^{*}\left[\left(f^{-1}\right)^{*} g\right]\right)= \\
\beta_{x}\left(f^{*}\left[\chi_{\eta_{n+1}(f(x))} \cdot\left(f^{-1 *} g\right)\right]\right)=\left[f_{*} \beta_{x}\right]\left(\chi_{\eta_{n+1}(f(x))} \cdot\left(f^{-1}\right)^{*} g\right)= \\
{\left[\theta \beta_{f(x)}\right]\left(\chi_{\eta_{n+1}(f(x))} \cdot\left(f^{-1 *} g\right)\right) .}
\end{gathered}
$$

Donde

$$
\left[b_{n}(g)\right](C)=\left[b_{n+1}\left(\theta\left(f^{-1}\right)^{*} g\right)\right](f C)
$$

Observa-se que $g \rightarrow \theta . g \circ f^{-1}$ é um automorfismo de $\overline{\mathcal{F}}^{+}(M)$ preservando a finitude. Além disso $C \rightarrow f C$ é um isomorfismo de Borel entre $M / \eta_{n} \rightarrow M / \eta_{n+1}$. Logo $b_{n+1}: \overline{\mathcal{F}}^{+}(M) \rightarrow$ $\overline{\mathcal{F}}^{+}\left(M / \eta_{n+1}\right)$ é um kernel que respeita a condição de finitude. Assim segue que $\eta_{n+1}$ satisfaz 4.6.

Assim prova-se o lema para $n>0$. Para $n<0$ repita o argumento com $f^{-1}$ ao invés de 
$f$.

Proposição 4.9. [16] A proposição 4.7 é válida para $G_{n}, \forall n \in \mathbb{Z}$. Além disso a coleção de medidas $\nu_{n}^{x}, x \in K$ define uma função transversa $\nu_{n}: \overline{\mathcal{F}}^{+}\left(G_{n}\right) \rightarrow \overline{\mathcal{F}}^{+}(M)$.

Demonstração. Substitui-se $\eta_{n}$ para $\eta=\eta_{0}$ na prova da proposição 4.7. A prova então segue, uma vez que o lema $4.8, \eta_{n}$ satisfaz as condições da proposição 4.3 e da proposição 4.6 . As quais são suficientes para provar o resultado para $\eta=\eta_{0}$.

Prova do teorema 4.5: [16] É provado primeiramente que tem-se uma função transversa em $G_{u, K}$. Dada $f \in \overline{\mathcal{F}}^{+}\left(G_{u, K}\right)$ pode-se decompô-la em

$$
f=\sum_{n \in \mathbb{Z}} f_{n}, f_{n} \in \overline{\mathcal{F}}^{+}\left(G_{n, K}\right) .
$$

Uma função $f_{n} \in \overline{\mathcal{F}}^{+}\left(G_{n}\right)$ pode ser considerada elemento de $\overline{\mathcal{F}}^{+}\left(G_{\infty}\right)$ ao se definir a mesma como zero no complementar de $G_{n}$ em $G_{\infty}$. Por 4.9 tem-se que para todo $n, \nu\left(f_{n}\right)=$ $\nu_{x}^{u}\left(f_{n}\right)=\nu\left(f_{n}\right) \in \overline{\mathcal{F}}^{+}$. Como $\nu_{n}\left(f_{n}\right)$ é mensurável para todo $n \in \mathbb{N}$ e $\sum \nu_{n}\left(f_{n}\right) \nearrow \nu(f)$ tem-se que $\nu(f)$ é mensurável.

É trivial verificar que $\nu$ é fibrado por $r$ e que $\nu$ é invariante pela ação de grupoide (pois $\nu_{u}$ é invariante).

A função transversa $\nu$ ser própria é equivalente a existir uma função não nula $f \in$ $\overline{\mathcal{F}}^{+}\left(G_{u, K}\right)$ tal que $\nu(f)$ é limitada. Porém sabe-se que para cada $\nu_{n}$ existe uma função não nula $f_{n} \in \overline{\mathcal{F}}^{+}\left(G_{n}\right)$ tal que $\nu_{n}(f)$ é limitada por uma constante $M(n)$. Defina portanto a função $f \in \overline{\mathcal{F}}^{+}\left(G_{u, K}\right)$ da seguinte maneira

$$
f=\sum_{i \in \mathbb{N}} \frac{f_{n}}{M(n) 2^{n}} \chi_{G_{n} \backslash \cup_{i=1}^{n-1} G_{i}}
$$

$\log 0$

$$
\nu(f)=\sum_{n \in \mathbb{N}} \frac{\nu_{n}\left(f_{n}\right)}{M(n) 2^{n}} \leq 1
$$

\subsection{Homomorfismo Jacobiano}

Conforme anunciado no inicio do capítulo nesta seção é definida a função modular que juntamente com a função transversa definida anteriormente fazem com que a condição $(i)$ do teorema 3.28 seja satisfeita toda vez que a medida $\mu$ seja SRB.

Definição 4.10. O mapa $\delta_{u}: G_{u} \rightarrow \overline{\mathbb{R}}^{+}$é definido por

$$
\delta_{u}(\gamma)=\prod_{i=1}^{\infty} \frac{J^{u}\left(f^{-i} s(\gamma)\right)}{J^{u}\left(f^{-i} r(\gamma)\right)}
$$


O objetivo desta seção é provar o seguinte teorema.

Teorema 4.11. [16] A restrição de $\delta_{u}$ ao subgrupoide $G_{u, K} \subset G_{u}$ é um homomorfismo entre o grupoide mensurável e o grupo multiplicativo real $\mathbb{R}_{+}^{*}$. Esta restrição de $\delta_{u}$ é denominada

\section{Homomorfismo Jacobiano .}

A prova segue os mesmos passos das provas anteriores, primeiramente faz-se para $G_{0}$ e então estende-se os resultados para $G_{u, K}$

Lema 4.12. [16] O mapa

$$
\begin{aligned}
\delta_{0}: G_{0} & \rightarrow \mathbb{R}_{+}^{*} \\
\gamma & \mapsto \delta_{u}(\gamma)
\end{aligned}
$$

é um homomorfismo mensurável no sentido definido em 3.24.

Demonstração. Primeiramente checa-se se a imagem de $\delta_{0}$ está em $\mathbb{R}_{+}^{*}$. Para isto escolhe-se um elemento $\gamma \in G_{0}$, denote $x=s(\gamma) \in M$ e $y=r(\gamma) \in M$. Utiliza-se o lema 6.1 .1 de Ledrappier e Young. Que fornece que para cada $\gamma \in G_{0}, 0<\delta_{0}(\gamma)<\infty$., donde $\gamma_{0}(\gamma) \in \mathbb{R}_{+}^{*}$.

Para demonstrar que o mapa $\delta_{0}$ é mensurável observa-se primeiramente que para cada $\gamma \in G_{0}, \ln \left(\delta_{0}(\gamma)\right)$ é um número real finito. Estuda-se a composição dos mapas

$$
\begin{aligned}
\ln \circ \delta_{0}: G_{0} & \rightarrow \mathbb{R} \\
\gamma & \mapsto \sum_{i=1}^{\infty} \ln \left[\frac{J^{u}\left(f^{-i}(s(\gamma))\right)}{J^{u}\left(f^{-i}(r(\gamma))\right)}\right]
\end{aligned}
$$

Define-se para cada $n \in \mathbb{Z}^{+}$o mapa

$$
\begin{aligned}
g_{n}: G_{0} & \rightarrow \mathbb{R} \\
\gamma & \mapsto \sum_{i=1}^{n+1} \ln \left(\frac{J^{u}\left(f^{-i} s(\gamma)\right)}{J^{u}\left(f^{-i} r(\gamma)\right)}\right)
\end{aligned}
$$

Então para cada $\gamma \in G_{0}$ tem-se que

$$
\lim _{n \rightarrow \infty} g_{n}(\gamma)=\ln \circ \delta_{0}(\gamma)
$$

Nota-se que cada $g_{n}$ é mensurável pois ao definir para cada $i \in \mathbb{Z}^{+}$as funções

$$
\begin{aligned}
& j_{r, i}=J^{u} \circ f^{-i} \circ r: G_{0} \rightarrow \mathbb{R}^{+} \\
& j_{s, i}=J^{u} \circ f^{-i} \circ s: G_{0} \rightarrow \mathbb{R}^{+}
\end{aligned}
$$

Que são composições de funções mensuráveis, donde são mensuráveis, segue que

$$
g_{n}=\sum_{i=1}^{n+1}\left(\ln \circ j_{s, i}-\ln \circ j_{r, i}\right)
$$

que é a soma de funções mensuráveis, donde é mensurável. Logo g é mensurável. E pela mensurabilidade da exponencial segue que 


$$
\delta_{0}=\exp \circ \ln \circ \delta_{0}
$$

é mensurável.

Para mostrar o homomorfismo, sejam $\gamma, \gamma^{\prime} \in G_{0}$ tais que $\gamma \gamma^{\prime}$ é bem definido, e seja $x=s\left(\gamma^{\prime}\right), y=r\left(\gamma^{\prime}\right)=s(\gamma),=r(\gamma),=r(\gamma)$ tem-se

$$
\begin{gathered}
\ln \left(\delta_{0}(\gamma)\right)+\ln \left(\delta_{0}\left(\gamma^{\prime}\right)\right)=\lim _{N \rightarrow \infty} \sum_{i=1}^{\infty} \ln \left(\frac{J^{u}\left(f^{-i}(y)\right)}{J^{u}\left(f^{-i}(z)\right)}\right)+ \\
\sum_{i=1}^{N} \ln \left(\frac{J^{u}\left(f^{-i}(x)\right)}{J^{u}\left(f^{-i}(y)\right)}\right)=\lim _{N \rightarrow \infty} \sum_{i=1}^{N} \ln \left(\frac{J^{u}\left(f^{-i}(x)\right)}{J^{u}\left(f^{-i}(z)\right)}\right)=\ln \left(\delta_{0}\left(\gamma \gamma^{\prime}\right)\right)
\end{gathered}
$$

Donde segue que $\delta_{0}$ é um homomorfismo em $\mathbb{R}_{+}^{*}$.

Prova do teorema $4.11[16]$

Como $K$ é subconjunto mensurável de H e tem-se que $G_{u, K}=G_{\infty, K}$. Para cada $\gamma \in G_{u, K}$, existe um inteiro positivo $p$, tal que $\gamma \in G_{p}$. Denote por F o mapa

$$
F=(f \times f): M \times M \rightarrow M \times M
$$

Pela definição da sequência de grupoides $\left\{G_{n}\right\}_{n \in \mathbb{N}}, F^{-l} G_{i}=G_{i-l}$, onde cada $G_{n}$ é considerado subconjunto de $M \times M$. Observemos que para cada $\gamma \in G_{u, K}$ se $p \in \mathbb{Z}^{+}$, escolhido como acima, então $\gamma \in G_{p, K}$ e $\operatorname{logo} F^{-p} \gamma \in G_{0}$.

Relaciona-se $\delta_{u}(\gamma)$ com $\delta_{u}\left(F^{-p} \gamma\right)$. Para isto define-se $x=s(\gamma)$ e $y=r(\gamma)$ e observa-se que

$$
\begin{gathered}
\delta_{u}\left(F^{-p} \gamma\right)=\prod_{i=1}^{\infty} \frac{J^{u}\left(f^{-(i+p)} x\right)}{J^{u}\left(f^{-(i+p)} y\right)}=\prod_{i=p+1}^{\infty} \frac{J^{u}\left(f^{-i} x\right)}{J^{u}\left(f^{-i} y\right)}= \\
{\left[\prod_{l=1}^{p} \frac{J^{u}\left(f^{-l} y\right)}{J^{u}\left(f^{-l} x\right)}\right] \prod_{i=1}^{\infty} \frac{J^{u}\left(f^{-i} x\right)}{J^{u}\left(f^{-i} y\right)}=\left[\prod_{l=1}^{p} \frac{J^{u}\left(f^{-i} y\right)}{J^{u}\left(f^{-i} x\right)}\right] \delta_{u}(\gamma)}
\end{gathered}
$$

Então para $\gamma \in G$

$$
\delta_{u}(\gamma)=\left[\prod_{i=1}^{\infty} \frac{J^{u}\left(f^{-i} x\right)}{J^{u}\left(f^{-i} y\right)}\right] \delta_{u}\left(F^{-p} \gamma\right)
$$

Para $p \in \mathbb{N}$ define-se $\delta_{p}$ como a restrição de $\delta_{u}$ a $G_{p}$. Foi mostrado que

$$
\delta_{p}=\left[\prod_{i=1}^{p} \frac{j_{s, l}}{j_{r, l}}\right]\left[\left(F^{-p}\right)^{*} \delta_{0}\right]
$$

Que é uma função finita e mensurável em $G_{p}$. Assim $\delta_{u}$ restrito a qualquer $G_{p}$ é mensurável. Como $G_{\infty}$ é união enumerável de $G_{p}$ 's é fácil ver que $\delta_{u}$ é mensurável em $G_{\infty}$ e também 
no subconjunto $G_{u, K} \subset G_{\infty}$. É também claro que $\delta_{u}$ é homomorfismo.

\subsection{Medidas Transversas e Medidas SRB}

Nesta seção será utilizado o teorema 3.23 para exibir a injeção entre medidas transversas com função modular $\delta_{u}$ e as medidas SRB do sistema $(M, f)$. Segue o principal teorema da seção.

Teorema 4.13. [16] Se a medida de probabilidade boreliana $\mu$ é SRB então o mapa

$$
\begin{aligned}
\Delta: \mathcal{E}^{+} & \rightarrow \overline{\mathbb{R}}^{+} \\
\nu * \lambda & \mapsto \mu\left(\lambda\left(\delta_{u}^{-1}\right)\right)
\end{aligned}
$$

é medida transversa com módulo $\delta_{u}$ no grupoide de medida $G_{u, K}$.

Como $\nu$ é função transversa fiel então pelo teorema 3.23 tem-se que para qualquer função transversa $\omega \in \mathcal{E}^{+}$existe kernel $\lambda$ tal que $\omega=\nu * \lambda$. Primeiramente procede-se a prova de 4.13 para as funções transversas $\nu_{n}$.

Proposição 4.14. [16] Seja $\mu$ medida $S R B$ em $M$, e $\nu_{n}$ função transversa em $G_{n}$ da proposição 4.9. Então para todo $h \in \overline{\mathcal{F}}^{+}\left(G_{n}\right)$

$$
\left(\mu \circ \nu_{n}\right) \tilde{h}=\left(\mu \circ \nu_{n}\right) \frac{1}{\delta} h
$$

Demonstração. Primeiramente representa-se o grupoide $G_{n}$ como segue: $\eta_{n}$ é partição mensurável de $M$, e portanto escreve-se

$$
M=\bigcup_{z \in M / \eta_{n}} \eta_{n}(z)
$$

com $\eta_{n}(z) \subset M$ elemento da partição correspondendo a $z$. Pode-se parametrizar $M$ pelas coordenadas $\left(t_{z}\right)$, com $z \in M / \eta_{n}$, e $t_{z} \in \eta_{n}(z)$. O grupoide de medida $G_{n}$ pode ser então escrito como

$$
G_{n}=\bigcup_{C \in M / \eta_{n}} \eta_{n}(z) \times \eta_{n}(z)
$$

$G_{n}$ pode ser então parametrizado pelas coordenadas $\left(z, t_{z}, t_{z}^{\prime}\right)$. Com essas coordenadas temos

$$
\begin{aligned}
& s:\left(z, t_{z}, t_{z}^{\prime}\right) \mapsto\left(z, t_{z}^{\prime}\right) \\
& r:\left(z, t_{z}, t_{z}^{\prime}\right) \mapsto\left(z, t_{z}\right) .
\end{aligned}
$$

A função transversa $\nu_{n}$ toma a forma

$$
\left[\nu_{n}(h)\right]\left(z, t_{z}\right)=\int h\left(z, t_{z}, t_{z}^{\prime}\right) d \beta_{z, t_{z}}\left(t_{z}^{\prime}\right)
$$


como $\beta_{z, t_{z}}$ é o mesmo para todo $t_{z} \in \eta_{n}(z)$, assim abrevia-se por $\beta_{z}$. Segue que

$$
\left[\nu_{n}(\tilde{h})\right]\left(z, t_{z}\right)=\int \tilde{h}\left(z, t_{z}, t_{z}^{\prime}\right) d \beta_{z}\left(t_{z}^{\prime}\right)=\int h\left(z, t_{z}^{\prime}, t_{z}\right) d \beta_{z}\left(t_{z}^{\prime}\right)
$$

e

$$
\left[\nu_{n}\left(\frac{1}{\delta} h\right)\right]\left(z, t_{z}\right)=\int\left[\frac{1}{\delta} h\right]\left(z, t_{z}, t_{z}^{\prime}\right) d \beta_{z}\left(t_{z}^{\prime}\right) .
$$

Como $\eta_{n}$ é partição mensurável, existe um sistema de medidas condicionais para $\mu$. Assim para toda função boreliana $w$ em $M$

$$
\int_{M} w d \mu=\int_{M / \eta_{n}}\left[\mu_{z}(w)\right] d \hat{\mu}(z)
$$

onde $\mu_{z}$ é uma medida de probabilidade suportada em $\eta_{n}(z)$, e $\hat{\mu}$ é a medida quociente. Para todo $g \in \overline{\mathcal{F}}^{+}\left(G_{n}\right)$ tem-se que

$$
(\mu \circ \nu) g=\int_{M / \eta_{n}} \int_{\eta_{n}(z)} \int g\left(z, t_{z}, t_{z}^{\prime}\right) d \beta_{z}\left(t_{z}^{\prime}\right) d \mu_{z}\left(t_{z}\right) d \hat{\mu}(z)
$$

Como $\mu$ é medida SRB $\log 0 \mu_{z} \ll \beta_{z}$, q.t.z, como ambas são $\sigma$-finitas segue que $\exists \rho$ função mensurável tal que

$$
d \mu_{z}=\rho d \beta_{z}
$$

e tem-se de (2.25) que

$$
\delta_{u}(\gamma)=\frac{\rho(r(\gamma))}{\rho(s(\gamma))}
$$

i.e.,

$$
\rho\left(t^{\prime}\right)=\rho(t) \frac{1}{\delta\left(t, t^{\prime}\right)}
$$

donde segue a igualdade

$$
d \beta_{z}\left(t^{\prime}\right) d \beta_{z}(t) \rho\left(t^{\prime}\right) h\left(t, t^{\prime}\right)=d \beta_{z}\left(t^{\prime}\right) d \beta_{z}\left(t^{\prime}\right) \rho(t) \frac{1}{\delta\left(t, t^{\prime}\right)} h\left(t, t^{\prime}\right)
$$

que implica

$$
d \mu_{z}\left(t^{\prime}\right) d \beta_{z}(t) h\left(t, t^{\prime}\right)=d \mu_{z}(t) d \beta_{z}\left(t^{\prime}\right)\left[\frac{1}{\delta} h\right]\left(t, t^{\prime}\right)
$$

donde integrando três vezes obtêm-se a equação (4.4).

Serget [16] não afirma nada sobre a volta da proposição 4.14. Porém em sua demonstração 
da mesma proposição faz parecer que a volta é válida, i.e. se a condição

$$
(\mu \circ \nu) \tilde{h}=(\mu \circ \nu) \delta^{-1} h
$$

é válida então $\mu$ é medida SRB. Para isso é necessário provar que

$$
\begin{gathered}
\iiint\left[\delta^{-1} h\right]\left(z, t_{z}, t_{z}^{\prime}\right) d \beta_{z}\left(t_{z}^{\prime}\right) d \mu_{z}\left(t_{z}\right) d \hat{\mu}(z)= \\
\iiint h\left(z, t_{z}^{\prime}, t_{z}\right) d \beta_{z}\left(t_{z}^{\prime}\right) d \mu_{z}\left(t_{z}\right) d \hat{\mu}(z)
\end{gathered}
$$

implica que $\mu_{z} \ll \beta_{z}$, para quase todo $z$. Suponha o contrário, i.e., existe conjunto $A$ de medida $\hat{\mu}$ positiva tal que para cada $z \in A, \exists A_{z}$ tal que $\mu_{z}\left(A_{z}\right)>0$ e $\beta_{z}\left(A_{z}\right)=0$. Caso $\chi_{\cup_{z \in A} A_{z} \times M}$ é função mensurável logo o lado direito da equação (4.5) é positivo e o lado esquerdo é nulo, uma contradição. Porém como $\cup_{z \in A} A_{z}$ é união não enumerável, logo não é necessariamente mensurável, e portanto $\chi_{\cup A_{z} \times M}$ não é necessariamente um função mensurável, e portanto não pode ser integrada.

Prova do teorema $4.13[16]$

Provou-se que se $\mu$ é medida SRB em $M$, então a condição $(i)$ do teorema 3.28 é satisfeita, i.e., $\forall g \in \overline{\mathcal{F}}^{+}\left(G_{u, K}\right)$

$$
(\mu \circ \nu) \tilde{g}=(\mu \circ \nu) \delta^{-1} g
$$

onde $\nu$ é a função transversa de Riemann, que é própria.

Utilize a decomposição de $g \in \overline{\mathcal{F}}^{+}\left(G_{u, K}\right)$ como

$$
g=\sum_{n \in \mathbb{Z}} g_{n}
$$

com cada $g_{n}$ suportado em $G_{u, K} \subset G_{u, K}$. Pode-se pensar em $g_{n}$ como pertencendo a $\overline{\mathcal{F}}\left(G_{n}\right)$. Assim pela proposição 4.14 equação (4.6) é verdadeira para cada $g_{n}$. Ao somar sobre $n \in \mathbb{Z}$, a equação (4.6) é verdadeira para $g$. Como $\nu$ é função transversa própria pode-se usar o teorema 3.28 para concluir que $\mu=\Delta_{\nu}$, e $\Delta$ é portanto medida transversa.

\subsection{Comentários Finais}

Conforme mencionado os resultados obtidos nesta capítulo são insuficientes para garantir uma bijeção entre as medidas transversas do grupoide $G_{u, K}$ e as medidas SRB de $(M, f)$. $\mathrm{O}$ que os resultados permitem garantir é que para cada medida SRB existe uma única medida transversa correspondente, assim caso exista uma única medida transversa deve existir no máximo uma única medida SRB. No geral se garante que a cardinalidade do conjunto de medidas SRB do sistema $(M, f)$ é sempre menor ou igual a cardinalidade de medidas transversas em $G_{u, K}$. 
A critica que apresenta-se a este método de estudo de medidas SRB é que medidas transversas não parecem ser simples. Conforme pode-se observar na última seção do capítulo 3 determinar a cardinalidade do conjunto de medidas transversas não é algo direto. Segert [16] enuncia, porém não mostra, que pode-se provar diretamente que o Cat Map (exemplo 2.25) tem uma única medida transversa. 


\section{Referências Bibliográficas}

[1] BARTLE R. G. The Elements of Integration and Lebesgue Measure. Estados Unidos: John Wiley \& Sons, 1995. 180p.

[2] COHN D. L. Measure Theory. Boston, Estados Unidos: Birkhöuser, 1980. 375p.

[3] CONNES A. Sur la Theorie Noncommutative de l'Integration. Lecture Notes in Mathematics, Berlin, v. 725, 1978.

[4] FOLLAND G. B. Real Analysis : modern techniques and their applications. Estados Unidos: John Wiley \& Sons, $2 \nmid$ edição, 1999. 386 p..

[5] HASSELBLATT B., PESIN Y. Nonuniform Hyperbolicity. Disponível em <http://www.scholarpedia.org/article/Nonuniform_hyperbolicity $>$. Acesso em 9 de Julho de 2013.

[6] EINSIEDLER M., WARD T. Ergodic Theory : with a view towards number theory. Londres, Inglaterra: Springer, 2011. 481 p..

[7] KASTLER D. On A. Connes' Noncommutative Integration Theory. Communications in Mathematical Physics, v. 85, 99-120, 1982.

[8] KURATOWISKI K., Topology. New York, New York: Academic Press, 1966. 580 p.

[9] LEDRAPPIER F., STRELCYN J. M. Estimation from bellow in Pesin's entropy formula, Ergodic Theory and Dynamical Systems, v.2, 203 (1982).

[10] LEDRAPPIER F., YOUNG L. S. The metric entropy of diffeomorphisms, Part I: Characterization of measures satisfying Pesin's entropy formula, Annals of Mathematics, v.122, 509 (1985).

[11] LIMA E. L. Elementos de Topologia Geral. Rio de Janeiro: SBM, primeira edição, 2009. $297 \mathrm{p}$.

[12] MACKEY G. W. Borel Structure in Groups and their Duals. Trans. Amer. Math. Soc. v. 85, 134-165, 1957.

[13] MUNKRES J. R. Topology. Estados Unidos: John Wiley \& Sons, 1995. 180p.

[14] OLIVEIRA K.; VIANA M. Teoria Ergódica: Um Curso Introdutório. Disponível em: <http://w3.impa.br/viana/out/ite.pdf>. Acesso em: 4 de maio de 2013.

[15] ROHLIN, V. A. On the Fundamental Ideas of Measure Theory. American Mathematical Society, 107 - 150, 1952. 
[16] SEGERT, J. Hyperbolic Dynamical Systems and the Noncommutative Integration Theory of Connes. 1987. 113 f.. Tese (Doutourado em Física) - Departamento de Física, Universidade de Princeton, Princeton, Nova Jersey, Estados Unidos.

[17] VIANA M. Disintegration into Conditional Measures: Rokhlin's Theorem. Disponível em: <http://w3.impa.br/viana/out/rokhlin.pdf>. Acesso em: 1 de maio de 2013.

[18] YOUNG L-S. What are SRB measures, and which dynamical systems have them. Journal of Statistical Physics, v. 108, 733-754, 2002. 


\section{Índice Remissivo}

G-kernel, 27

$\sigma$-álgebra, 3

enumeravelmente gerada, 4

gerada, 3

produto, 4

alvo, r, 22

Arnold's Cat Map, 18

atomo, 12

conjunto de pares multiplicáveis, 22

conjunto mensurável, 3

desintegração, 10

espaço

de medida, 4

de probabilidade, 4

mensurável, 3

polonês, 6

standart, 6

esperança condicional, 11

expoente de Lyaponov, 19

fonte, s, 21

função

integrável, 6

característica, 5

indicadora, 5

mensurável, 4

modular, 34

simples, 5

transversa, 33

grupoide, 21

de medida, 24

associado, 24

compátivel, 25

instável, 45

principal, 22

principal associado, 22, 24

principal compatível, 25 homomorfismo, 34

Jacobiano, 51

igualdade $\bmod 0,5$

isomorfismo mensurável, 4

kernel, 26

$\sigma$-finito, 27

carregado, 26

fibrado, 26

fiel, 30

próprio, 27

medida, 4

de probabilidade, 4

regular, 5

SRB, 18

absolutamente contínua, 6

externamente regular, 4

internamente regular, 5

invariante, 17

transversa, 34

partição, 7

crescente, 45

mensurável, 7

refinada, 7

produto tensorial, 40

quase sempre, q.s, 5

quase todo ponto, q.t.p., 5

sistema de medidas condicionais, 10

sistema dinâmico, 16

hiperbólico, 20

uniformemente hiperbólico, 17

suporte de um kernel, 30

taxa de expansão da variedade, 17

Teorema

da Convergência Dominada, TCD, 6

da Convergência Monótona, TCM, 5

da Desintegração de Rohklin, 13 